\title{
Taking the Cloth: How Religious Appeals Increase Compliance with COVID-19 Prevention Measures
}

\author{
Claire L. Adida $^{\dagger^{*}}$, Christina Cottiero ${ }^{\dagger}$, Leonardo Falabella ${ }^{\dagger}$, Isabel Gotti ${ }^{\dagger}$, ShahBano Ijaz $^{\dagger}$, \\ Gregoire Phillips ${ }^{\dagger}$, and Michael Seese ${ }^{\ddagger}$ \\ ${ }^{*} P I$ and Corresponding Author. Email: cadida@ucsd.edu \\ ${ }^{\dagger}$ Department of Political Science, University of California San Diego \\ ${ }^{\ddagger}$ School of Global Policy and Strategy, University of California San Diego
}

January 29, 2021 


\begin{abstract}
During the COVID-19 pandemic, face masks have been recommended by the CDC and the WHO as key to reducing viral transmission. Yet, in the United States, one fifth of individuals say they wear masks at most some of the time, and a majority say that people in their community wear masks at most some of the time. What strategies most effectively encourage compliance with this critical COVID-19 prevention measure? Relying on social identity theory, we experimentally assess two possible mechanisms of compliance, elite endorsement and social norms, among a representative sample of White U.S.-born Evangelicals, a group that has shown resistance to prevention measures. We find evidence for both mechanisms, but social norms play a remarkably important role - increasing support for mask-wearing by $6 \%$ with spillover effects on other prevention guidelines. Our findings confirm the role that appeals to norms and elite endorsements play in shaping individual behavior, and offer lessons for public health messaging.
\end{abstract}

Keywords: COVID-19; Norms; Political communication; Public Health; Religion; Social identity; Survey experiment 


\section{Introduction}

As of 6 January 2021, the COVID-19 pandemic has claimed over 1.86 million lives and infected more than 86 million people worldwide, putting this public health emergency on track to be the most deadly in over a century. ${ }^{1}$ A striking feature of the ongoing pandemic is the absence of clear and consistent public health recommendations by local and national governments. In the United States, for example, the Centers for Disease Control and Prevention recommends such measures as hand washing, physical distancing, and mask wearing, but local jurisdictions throughout the country do not uniformly condone or enforce these policies. Former U.S. President Donald Trump has himself provided inconsistent messages on the necessity and efficacy of these measures.

The scientific consensus on the effectiveness of face masks in preventing the spread of the virus (Anfinrud et al. 2020; Lyu and Wehby 2020) contrasts with contentious reactions across the US political landscape. A majority of Americans report that they wear face masks in public, at most, "some of the time". Strikingly, although $65 \%$ of Americans claim to wear a mask when in stores or businesses all or most of the time, only $44 \%$ of Americans say that others in the community where they live do so (Igielnik 2020). Today, wearing a mask has become a partisan issue (Gadarian, Wallace, and Pepinsky 2020; Milosh et al. 2020).

Recent work in the behavioral, social, and psychological sciences informs us that while this defiance of public health guidelines may seem puzzling on its face, it is strikingly consistent with social identity theories of behavior in which individuals prioritize the self-esteem they receive from belonging to a social group. Scholars have shown just how powerful social identity is in guiding individuals' actions and beliefs, finding that when processing and integrating new information, individuals seek not accuracy, but rather affirmations of themselves as members of a social group. This justification helps us understand motivated reasoning, or the process by which individuals seek out and more readily accept information that confirms

\footnotetext{
${ }^{1}$ World Economic Forum, "COVID-19: What you need to know about the coronavirus pandemic on 6 January"; accessed online on 6 January 2021. Available at: https://www.weforum.org/platforms/ covid-action-platform/articles/covid-19-coronavirus-pandemic-6-january.
} 
their priors (Kahan 2016). From this perspective, the partisan divide over face masks is not so much a puzzle, but a characteristic of the country's intense partisan polarization.

In this paper, we draw on these insights to test how social identity can be leveraged to encourage individual compliance with public health guidelines in a pandemic. Specifically, relying on a survey experiment of White Evangelical Americans - one of the least compliant groups in the country (Burge 2020; PRRI 2020) - we test two mechanisms that guide behavior within social identity groups: the influence of in-group elites, and the powerful role of ingroup norms. Limiting our tests to a single social identity group allows us to hold several other factors of cross-group analysis constant in order to examine precisely how social identity might shape individual compliance.

The influence of religious elites on individual attitudes and behavior is well-documented in the social science literature. Scholars find that religious elites play an outsized role in political participation (McClendon and Riedl 2019), voting behavior (Green 2010), and compliance with public health directives (Muñoz-Laboy et al. 2011). ${ }^{2}$ Alternatively, individuals may take a cue not from their religious leader but from what they perceive to be acceptable norms of behavior within their religious group. Ingroup norms are a powerful motivator of individual behavior in many other contexts (Paluck and Shepherd 2012). Do they operate similarly in the context of compliance with COVID-19 prevention measures?

We test these hypotheses with a survey experiment on a representative sample of U.S.born White Evangelicals fielded in July 2020. Individuals are either assigned to a control condition in which they receive basic public health recommendations to prevent COVID-19, an elite condition in which they receive the same recommendations plus a quote from an Evangelical leader (Reverend Franklin Graham) encouraging mask-wearing, or a norms condition in which they receive the same recommendations plus a statement about the percentage of White Evangelicals who have worn a mask in public (close to 80\%). We then measure respondents' attitudinal, semi-behavioral, and behavioral support for mask wearing.

\footnotetext{
${ }^{2}$ Some research suggests that Evangelical Christians may not respond to elite cues when these are overtly political or when they come into tension with an overlapping, partisan, identity (Margolis 2018).
} 
Both our elite and norms treatments significantly increased White Evangelicals' expressed support for mask wearing in public. However, it is our social norms treatment that had the most consistently statistically significant effect. Not only did it increase expressed support for mask wearing in public, it also increased support for social and physical distancing as a prevention measure; further, it increased respondents' likelihood of saying they would be willing to sign a petition endorsing mask-wearing in public. We additionally find that respondents whose religious motivation is their social identity - those who tell us their Church is important to them first and foremost because they identify as Evangelical Christian respond more strongly to the norms treatment, and that the norms treatment was more likely to convey new information to our respondents than was the elite endorsement. These results clarify how social identity shapes individual compliance with public health recommendations.

\section{The Power of Social Identity}

Our theoretical intuition draws from Social Identity Theory, which argues that individuals want to maximize their self-esteem and do so by favoring the social groups to which they belong (Tajfel and Turner 1979). Social scientists have relied on this intuition to better understand ingroup bias in a number of contexts, from voting (e.g., Bassi, Morton, and Williams 2011), to partisan identity formation (e.g., Green, Palmquist, and Schickler 2004), to policy preferences (e.g., Shayo 2009). We build on these insights by interrogating which audiences individuals care about within these groups. Although much of the literature on social norms assumes that individuals care about their peers, another literature on elite endorsements suggests that individuals may care about their group leaders (e.g., Zaller 1992).

We test these intuitions in the context of compliance with mask-wearing and social distancing protocols to prevent the spread of COVID-19. More specifically, we examine White Evangelicals in the United States, a social group whose religious identity is highly salient especially as it compares to that of White Americans of other religions (Putnam and Camp- 
bell 2006) - and one that is known for refusing to comply with COVID-19 health guidelines (Burge 2020; Smith 2020; PRRI 2020). Our study tests the below hypotheses:

Hypothesis $\mathbf{H}_{\mathbf{1}}$ : White Evangelicals are more likely to support wearing facemasks in public when they receive an ingroup elite endorsement of wearing facemasks in public.

Hypothesis $\mathbf{H}_{2}$ : White Evangelicals are more likely to support wearing facemasks in public when they receive information that a majority of their peers wear facemasks in public.

In July 2020, we fielded a survey experiment with the Lucid marketplace on a sample of 1,780 White Evangelical American adults. ${ }^{3}$ Although a random sample of White Evangelicals - while ideal - is prohibitively costly, we used quotas on gender, age, and US region to match the demographic characteristics of US White Evangelicals according to the Pew Research Center. ${ }^{4}$

The survey collects a number of pretreament covariates, assigns the treatment, and measures support for wearing facemasks in public. We test Hypotheses $\mathrm{H}_{1}$ and $\mathrm{H}_{2}$ via random assignment to one of three conditions. In the control condition, respondents read a short vignette describing a few facts about COVID-19 transmission. These facts are taken from the $\mathrm{CDC}$ website. In the endorsement condition testing $\mathrm{H}_{1}$, this same set of facts was presented with the addition of a statement by Evangelical leader Franklin Graham endorsing maskwearing. In the social norms condition testing $\mathrm{H}_{2}$, the endorsement statement was replaced by the statement that close to $80 \%$ of White Evangelicals have worn a mask in public. SI-A provides a screenshot of each condition. Our source for Pastor Graham's statement is a 10 April 2020 interview with the Charlotte Observer. Our source for the statistic that $80 \%$ of White Evangelicals have worn a mask in public comes from the magazine Christianity

\footnotetext{
${ }^{3}$ Recent research has shown that online survey experiments during the COVID-19 pandemic do not suffer from significant changes in how subjects respond to treatments, and possibly generate smaller average treatment effects due to increased inattentiveness (Peyton, Huber, and Coppock 2020). This would make it more difficult to detect an effect that exists.

${ }^{4}$ We relied on a series of screening questions at the beginning of our survey to obtain a sample of US Born White Evangelicals: respondents qualified if they responded that they are not of Hispanic, Latino, or Spanish origins, that they are White, that they are Protestant, that they are an Evangelical Christian, and that they are a US citizen.
} 
Today, which cites a COVID-19 Tracking Poll from Data for Progress. For each vignette, we also asked respondents whether they already knew the information, whether they did not know it, or whether they did not believe it. We use this question as a measure of compliance with treatment, identifying non-compliers as those who answered that they do not believe the information.

We measure four outcomes, ranging from attitudinal to behavioral. A thermometer question asks, on a scale of 0 to 10, how important the respondent believes wearing a mask in public is for combatting the spread of COVID-19. This is the outcome measure we use for our power analysis. A second question asks the extent to which, on a scale from 1 ("Strongly disagree") to 5 ("Strongly agree"), the respondent agrees with a set of policies to mitigate the spread of COVID-19. Finally, a behavioral measure asks respondents how likely, on a scale of 0 to 10, they would add their name to a Change.org petition endorsing mask-wearing in public. If respondents answer 5 or higher, they are then given the option to click on the petition URL, which is a decision we can track.

\section{Results}

Our sample consists of 1,780 U.S.--born White Evangelicals, 1,066 (59.89\%) of whom identify as female, $713(40.06 \%)$ as male, and 1 as non-binary. The mean age in our sample is 54.23 years and roughly $68 \%$ of the sample identify as Republican, $16 \%$ identify as Democrats, and the remaining $16 \%$ as Independents (SI-B). According to a 2015 Pew Research Poll, the Evangelical population writ large has a median age of 49 , is $55 \%$ female-identifying, and is 56\% Republican (Pew Research Center 2015).

Who in our U.S.-born White Evangelical sample is more likely to wear a mask to begin with? We know that, among the US population more broadly, women, racial minorities, older individuals, and Democrats are more likely to support mask-wearing in public to prevent the spread of the virus (Pew Research Center 2020). Our correlational analysis in SI-C 
shows that older, more educated, and less Republican respondents are more likely to agree with the importance of mask-wearing in public; these patterns hold in a multiple regression framework.

We test hypotheses $\mathrm{H}_{1}$ and $\mathrm{H}_{2}$ with the below pre-registered specification:

$$
y_{i}=\alpha+\beta T_{i}+\gamma X_{i}+\epsilon_{i}
$$

where $y_{i}$ is the outcome of interest for respondent $i, \beta$ is the estimated treatment effect, $T_{i}$ is the assignment to treatment, $X_{i}$ is a vector of demographic covariates, and $\epsilon_{i}$ is the error term. In our pre-analysis plan, we did not specify which demographic covariates to include in $X_{i}$. We therefore include all pretreatment demographic controls.

Figure 1 illustrates our main results testing the elite endorsement and the norm conditions relative to the control condition (see SI-D for regression tables). It indicates a significant effect of our elite endorsement condition on a single outcome measure: the thermometer score expressing support for wearing masks in public. And yet, for nearly every other outcome measure that follows, the treatment effect is no longer statistically distinguishable from zero.

By contrast, the social norms message shows more statistically significant effects across a variety of outcomes. Relative to the control condition conveying solely the CDC guidelines at the time, the norms condition increases the respondent's thermometer score in support of mask-wearing in public; it also increases agreement with both mask-wearing and social distancing as effective policies. Finally, though the effect is statistically significant only at the $90 \%$ confidence level, the norms message increases the respondent's expressed willingness to sign a petition endorsing mask-wearing in public.

Substantively, the average treatment effects we uncover in both conditions are consistent with similar studies on the determinants of mask-wearing and social distancing during COVID19 (e.g., Rieger 2020). Our norms message increases respondents' thermometer scores by 0.15 standard deviations on average, while the endorsement message increases thermometer scores by 0.11 standard deviations. 


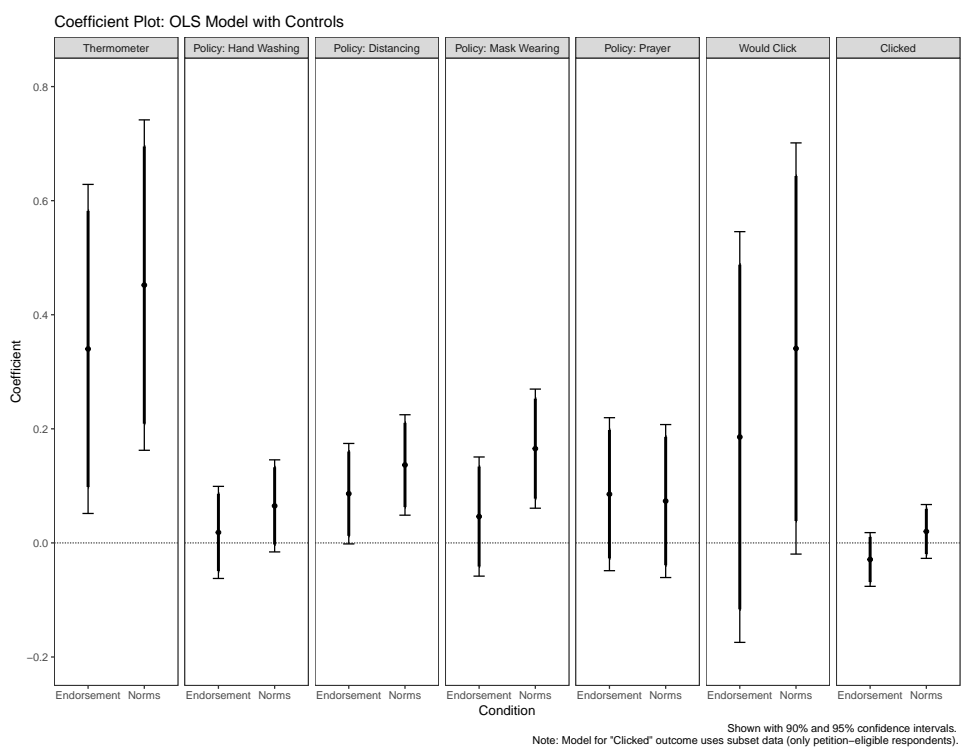

Figure 1: Average Treatment Effects

Our pre-analysis plan also pre-registered a compliance analysis. After each treatment condition, we asked respondents to tell us whether they already knew the information, or whether they did not believe it. We then coded those who responded "I do not believe this" as non-compliers and conduct a complier average causal effect (CACE) analysis in SI-E. Our results hold. Finally, we registered a series of tests to investigate the social identity mechanism we posit in this paper: if White Evangelicals can be moved to express greater support for face masks through a social identity mechanism, then we should expect respondents who identify as White Evangelical because it is their social identity to respond more strongly to our treatments. In SI-G, we find that they do. ${ }^{5}$

Although our treatment effects are not statistically significantly different from one another, there is one crucial way in which they differed. In SI-H, we show that respondents were less likely to already know the information provided by our norms treatment (49\%) than that provided by our elite treatment (81\%). If "individuals are motivated to understand what is normative in the communities to which they belong" (Tankard and Paluck 2016), then changing a White Evangelical's perception about what is normative in their community may

\footnotetext{
${ }^{5}$ In SI-F, we show that our treatment effects are not mediated by religiosity or religious motivation.
} 
have influenced their attitude toward mask-wearing.

\section{Conclusion}

In this paper, we draw from social identity theory to test how White Evangelicals, a group that has been less compliant with COVID-19 prevention guidelines, can be persuaded to follow a key health recommendation: wearing masks in public. Our results indicate that both leader and peer audiences effectively shape compliant attitudes toward mask-wearing, with more wide-ranging effect for the peer norms treatment. Additionally, our results show that White Evangelicals did not know the extent to which their peers were already abiding by this norm; by informing them of this, our vignette shaped perceptions around the existence of this norm, motivating respondents to comply with it as well.

The effect of our elite vignette was less robust. Research has shown that the influence of religious elites has its limits, particularly when elite positions deviate significantly from those of group members on polarizing issues such as the environment or LGBT rights (e.g., Djupe and Claassen 2018). In particular, Margolis (2018) shows that cross-pressures between Evangelical Christians' Evangelical and Republican identities can be demobilizing. Alternatively, White Evangelicals may seek out the view of their local religious leaders, rather than that of national evangelical leaders (Djupe and Claassen 2018). As a result, our vignette may have provided a cue from the "wrong" elite; future work should test whether cues from more local Evangelical leaders are more effective.

Our findings have important implications for public health messaging, suggesting that White Evangelicals may be more receptive to public health messaging when it is delivered by in-group leaders. Our findings suggest, however, that it is most important for public messaging to dispel myths around compliance among White Evangelicals. When White Evangelicals learn that many members of their group do, in fact, comply with mask wearing and other preventative measures, they may be more willing to follow suit. 


\section{$5 \quad$ References}

Anfinrud, Philip et al. (2020). "Visualizing Speech-Generated Oral Fluid Droplets with Laser Light Scattering." In: New England Journal of Medicine 382.21, pp. 2061-2063.

Bassi, A., R.B. Morton, and K.C. Williams (2011). "The effects of identities, incentives, and information on voting." In: Journal of Politics 73, pp. 558-71.

Burge, Ryan P. (June 23, 2020). "White Evangelicals' Coronavirus Concerns Are Fading Faster." In: Christianity Today.

Djupe, Paul and Ryan L. Claassen (2018). The Evangelical Crackup?: The Future of the Evangelical-Republican Coalition. Philadelphia: Temple University Press.

Gadarian, S., S. Goodman Wallace, and T.B. Pepinsky (2020). "Partisanship, health behavior, and policy attitudes in the early stages of the COVID-19 pandemic." In: $S S R N$ Working Paper.

Green, D.P., B. Palmquist, and E. Schickler (2004). Partisan Hearts and Minds: Political Parties and the Social Identities of Voters. New Haven: Yale University Press.

Green, John C. (Feb. 25, 2010). "Gauging the God Gap: Religion and Voting in US Presidential Elections." In: The Oxford Handbook of American Elections and Political Behavior.

Igielnik, Ruth (2020). Most Americans say they regularly wore a mask in stores in the past month; fewer see others doing it.

Kahan, Dan M. (2016). "Emerging trends in the social and behavioral sciences." In: ed. by Robert Scott and Stephen Kosslyn. John Wiley \& Sons, Inc.

Lyu, Wei and G. L. Wehby (2020). "Community use of face masks and COVID-19: Evidence from a natural experiment of state mandates in the US." In: Health Affairs 39.8.

Margolis, Michele F. (2018). "How far does social group influence reach? Identities, elites, and immigration attitudes." In: The Journal of Politics 80.3.

McClendon, Gwyneth H. and Rachel Beatty Riedl (Nov. 14, 2019). From Pews to Politics: Religious Sermons and Political Participation in Africa. Cambridge University Press. 
Milosh, Maria et al. (2020). "Unmasking partisanship: polarization undermines public response to collective risk." In: Becker Friedman Institute for Economics 2020-102.

Muñoz-Laboy, Miguel et al. (Oct. 1, 2011). "Religious Responses to HIV and AIDS: Understanding the Role of Religious Cultures and Institutions in Confronting the Epidemic." In: Global Public Health 6 (sup2), S127-S131. pmid: 21806421.

PRRI (2020). "The Coronavirus Pandemic's Impact on Religious Life." In:

Paluck, Elizabeth Levy and Hana Shepherd (2012). "The Salience of Social Referents: A Field Experiment on Collective Norms and Harassment Behavior in a School Social Network." In: Journal of Personality and Social Psychology 103.6, pp. 899-915.

Pew Research Center (2015). America's Changing Religious Landscape.

- (2020). Republicans, Democrats move even further apart in Coronavirus concerns.

Peyton, Kyle, Gregory A. Huber, and Alexander Coppock (2020). "The generalizability of online experiments conducted during the COVID-19 pandemic." In: SocArXiv.

Putnam, Robert D. and David E. Campbell (2006). Faith Matters Survey. Ann Arbor, MI: Inter-university Consortium for Political and Social Research.

Rieger, Marc. (2020). "To wear or not to wear? Factors influencing wearing face masks in Germany during the COVID-19 pandemic." In: Social Health and Behavior 3.2.

Shayo, M. (2009). "A model of social identity with an application to political economy: nation, class, and redistribution." In: American Political Science Review 103.

Smith, Gregory A. (2020). "Most white evangelicals satisfied with Trump's initial response to the COVID-19 outbreak." In: Pew Research Center.

Tajfel, H. and J.C. Turner (1979). "The social psychology of intergroup relations." In: ed. by W.G. Austin and S. Worchel. Monterey, CA: Brooks/Cole, pp. 33-47.

Tankard, M. and E.L. Paluck (2016). "Norm perception as a vehicle for social change." In: Social Issues and Policy Review 10.1.

Zaller, John (1992). The Nature and Origins of Mass Opinion. New York: Cambridge University Press. 


\section{Supplementary Information}

Taking the Cloth: How Religious Appeals Increase Compliance with COVID-19 Prevention Measures 


\section{Appendices}

\section{Contents}

Appendices 20

A Treatment vignettes 3

B Summary Statistics \& Balance 4

C Correlates of Mask Wearing 11

D OLS Analyses: Average Treatment Effects 14

E Compliance Analyses: Compliance Average Causal Effects 17

F Mediation Analyses $\mathbf{2 0}$

F.1 Religiosity (Self-Reported) . . . . . . . . . . . . . . . . . . . . . 20

F.2 Religious Motivation . . . . . . . . . . . . . . . . . . . . . . . . 22

F.3 Extrinsic/Intrinsic Motivations . . . . . . . . . . . . . . . . . . . . 29

F.4 Religiosity Index . . . . . . . . . . . . . . . . . . . . . . . . . . 33

G Interaction Models $\quad 37$

H Who already knew our treatment information? 59

I Pre-analysis plan $\quad \mathbf{6 0}$ 


\section{A Treatment vignettes}

\section{Did you know?}

Covid-19 mainly spreads through droplets exhaled by infected individuals when they talk, cough, or sneeze.

If a healthy individual inhales these droplets, they can easily become infected with Covid-19.

To prevent the spread of Covid-19, the US government recommends that all people over the age of two wear masks to cover their mouths and noses in public places.

I already knew this

I did not already know this

$\bigcirc$ I do not believe this

(a) Control Condition

Did you know?

Covid-19 mainly spreads through droplets exhaled by infected individuals when they talk, cough, or sneeze.

If a healthy individual inhales these droplets, they can easily become infected with Covid-19.

To prevent the spread of Covid-19, the US government recommends that all people over the age of two wear masks to cover their mouths and noses in public places.

In a recent interview, the Evangelical leader Franklin Graham agreed: ''We want to be careful,' Graham said, and we want to protect other people. And I think the social distancing is going to help. I think people wearing masks is a huge help."'

I already knew this

I did not already know this

I do not believe this

(b) Elite Condition

Did you know?

Covid-19 mainly spreads through droplets exhaled by infected individuals when they talk, cough, or sneeze

If a healthy individual inhales these droplets, they can easily become infected with Covid-19.

To prevent the spread of Covid-19, the US government recommends that all people over the age of two wear masks to cover their mouths and noses in public places.

Today, close to $80 \%$ of White Evangelicals wear masks in public.

I already knew this

I did not already know this

$O$ I do not believe this

(c) Norms Condition

Figure A1: Treatment Conditions 


\section{B Summary Statistics \& Balance}

The variables in this study are labeled and described below:

- Age: "What is your year of birth?"

- Follows News: "How frequently do you seek out news and information on current affairs?" with options: Less than once a month; A few times a month; A few times a week; Once a day; More than once a day.

- Follows Covid: "How frequently do you seek out news and information on the ongoing COVID-19/coronavirus situation?" with options: I do not follow this situation at all; Rarely; Sometimes; Often; All the time.

- Covid Threat: "Would you say that the coronavirus outbreak is a major threat, a minor threat, or not a threat to your personal health?" with options: A major threat; A minor threat; Not a threat.

- Gender: "What is your gender?" with options: Male; Female; Non-binary; Other (openended)

- Education: "What is the highest grade or level of school that you have completed?" with options: 8th grade or less; High school but no diploma; High school diploma or equivalent (GED); Vocational diploma after high school; Some college but no degree; Associate's degree (AA, AS); Bachelor's degree (BA, BS); Some graduate or professional education but no degree; Master's degree (MA, MS); Doctorate degree (PhD, EdD); Professional degree beyond bachelor's degree (MD, DDS, JD, LLB).

- Party ID: "In general, would you describe yourself as a:" with options: Strong Democrat; Democrat; Lean Democrat; Independent; Lean Republican; Republican; Strong Republican.

- News Source: "What is your primary source of news on current affairs?" with options: I don't consume news/current affairs; Television; Newspapers or news magazines; Social media such as Facebook, Twitter, Youtube, Instagram, etc...; Internet news websites such as CNN, BBC, Fox news, etc...; Discussion boards and social news aggregators such as Reddit or 4chan; Podcasts; Friends or family; Community leader; Religious leader.

- Covid News Source: "What is your primary source of news on COVID-19/coronavirus?" with options: I don't consume news/current affairs; Television; Newspapers or news magazines; Social media such as Facebook, Twitter, Youtube, Instagram, etc...; Internet news websites such as CNN, BBC, Fox news, etc...; Discussion boards and social news aggregators such as Reddit or 4chan; Podcasts; Friends or family; Community leader; Religious leader.

- Tested for Covid: "Have you personally been tested for COVID-19?" with options: Yes; No.

- Friends with Covid: "Have any of your family, friends, or acquaintances been tested for or diagnosed with COVID-19?" with options: Yes; No

- Religiosity (self-reported): "Please evaluate your own feelings of religiousness: are you" with options: Very religious; Moderately religious; Slightly religious; Not at all religious; Anti-religious. 
- Religiosity index: An additive index for each of the following three statements, for which respondents filled out to what extent they Strongly disagree; Disagree; Somewhat disagree; Somewhat agree; Agree; Strongly agree:

1. Regularly attends Church: I go to Church regularly.

2. Spiritual values: Spiritual values are more important than material things.

3. Americans should be more religious: If Americans were more religious, this would be a better country.

- Extrinsic/Intrinsic motivation index: A set of three statements, for which respondents filled out to what extent they Strongly disagree; Disagree; Somewhat disagree; Somewhat agree; Agree; Strongly agree. We treat each statement separately, we measure the extent of agreement.

1. Religion offers comfort: What religion offers me most is comfort in times of trouble and sorrow.

2. Takes religious approach to life: My whole approach to life is based on my religion.

3. Enjoys social aspects of Church: I go to church mainly because I enjoy seeing people I know there.

- Religious motivation variables: "Please rank the statements below, from statement you most agree with (at the top) to statement you least agree with (at the bottom)". Each individual is then coded by which statement they ranked first.

- Insurance: My Church is important to me because it helps me in times of personal or financial need.

- Morality: My Church is important to me because following the advice of my Church is the right thing to do.

- Social capital: My Church is important to me because that is where I find my family, close friends, and/or business partners.

- Heuristics: My Church is important to me because it makes it easier for me to reach decisions about complicated things.

- Tradition: My Church is important to me because I was born into it and/or brought up this way.

- Social identity: My Church is important to me because I identify as Evangelical Christian.

- Thermometer: "On a scale of 1 to 10, where 1 means "not at all important" and 10 means "absolutely important", how important do you think wearing masks in public is for combatting the spread of COVID-19/coronavirus?"

- Policy outcomes: "Please tell us the extent to which you agree with the below statements:" with options: Strongly disagree: Disagree: Neither agree nor disagree; Agree; Strongly agree.

- Hand washing: "Washing hands is an effective way to prevent the spread of COVID19/coronavirus."

- Distancing: "Social and physical distancing is an effective way to prevent the spread of COVID-19/coronavirus." 
- Mask wearing: "Wearing a mask is an effective way to prevent the spread of COVID19/coronavirus."

- Prayer: "Prayer is an effective way to prevent the spread of COVID-19/coronavirus."

- Would click: "On a scale of 1 to 10 where 1 means "Not at all" and 10 means "Absolutely", how willing are you to sign a petition endorsing mask-wearing in public?"

- Clicked: "In the previous question, you answered a 5 or above: if interested, here is a link to a Change.org petition endorsing mask-wearing in public: https://www. change.org/p/g overnment-wearing-face-masks-in-public-should-be-made-mandatory-by-the-gov ernment-to-prevent-covid-19

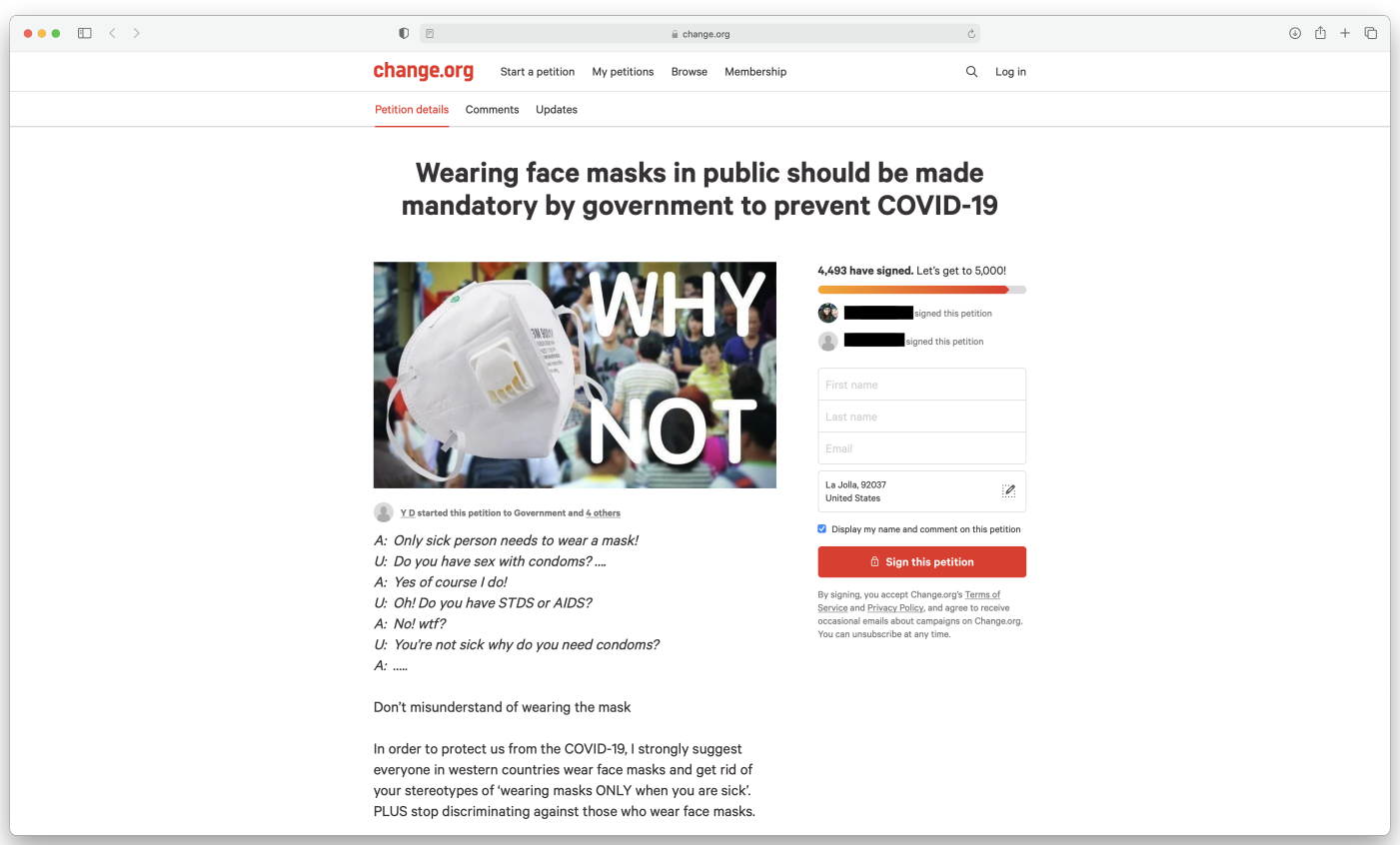

Figure B2: Change.org Petition 


\begin{tabular}{|c|c|c|c|c|c|c|c|}
\hline Variable & & $n$ & $\bar{x}$ & $s$ & Min & Mdn & Max \\
\hline Age & & 1778 & 54.23 & 15.92 & 18.00 & 57.00 & 80.00 \\
\hline Follows News & & 1780 & 3.72 & 1.20 & 1.00 & 4.00 & 5.00 \\
\hline Follows Covid & & 1779 & 3.78 & 0.93 & 1.00 & 4.00 & 5.00 \\
\hline Covid Threat & & 1780 & 2.46 & 0.62 & 1.00 & 3.00 & 3.00 \\
\hline Variable & Value & $n$ & $\%$ & & & & \\
\hline \multirow[t]{3}{*}{ Gender } & Female & 1066 & 59.89 & & & & \\
\hline & Male & 713 & 40.06 & & & & \\
\hline & Non-binary & 1 & 0.06 & & & & \\
\hline \multirow[t]{11}{*}{ Education } & $<8$ th Grade & 1 & 0.06 & & & & \\
\hline & $<$ High School & 24 & 1.35 & & & & \\
\hline & High School & 243 & 13.65 & & & & \\
\hline & Some College & 334 & 18.76 & & & & \\
\hline & Vocational School & 65 & 3.65 & & & & \\
\hline & Associate's & 223 & 12.53 & & & & \\
\hline & Bachelor's & 448 & 25.17 & & & & \\
\hline & Some Graduate School & 60 & 3.37 & & & & \\
\hline & Master's & 321 & 18.03 & & & & \\
\hline & Professional Degree & 32 & 1.80 & & & & \\
\hline & Doctorate & 29 & 1.63 & & & & \\
\hline \multirow[t]{7}{*}{ Party ID } & Strong Democrat & 95 & 5.34 & & & & \\
\hline & Democrat & 110 & 6.18 & & & & \\
\hline & Lean Democrat & 77 & 4.33 & & & & \\
\hline & Independent & 294 & 16.52 & & & & \\
\hline & Lean Republican & 247 & 13.88 & & & & \\
\hline & Republican & 407 & 22.87 & & & & \\
\hline & Strong Republican & 550 & 30.90 & & & & \\
\hline \multirow[t]{10}{*}{ News Source } & Community Leader & 8 & 0.45 & & & & \\
\hline & Discussion Boards and Aggregators & 15 & 0.84 & & & & \\
\hline & Friends or Family & 78 & 4.38 & & & & \\
\hline & Does Not Follow News & 42 & 2.36 & & & & \\
\hline & Internet News Sites & 454 & 25.51 & & & & \\
\hline & Newspapers or Magazines & 78 & 4.38 & & & & \\
\hline & Podcasts & 25 & 1.40 & & & & \\
\hline & Religious Leaders & 8 & 0.45 & & & & \\
\hline & Social Media & 235 & 13.20 & & & & \\
\hline & TV & 837 & 47.02 & & & & \\
\hline \multirow[t]{10}{*}{ Covid News Source } & Community Leader & 13 & 0.73 & & & & \\
\hline & Discussion Boards and Aggregators & 11 & 0.62 & & & & \\
\hline & Friends or Family & 57 & 3.20 & & & & \\
\hline & Does Not Follow News & 30 & 1.69 & & & & \\
\hline & Internet News Sites & 441 & 24.78 & & & & \\
\hline & Newspapers or Magazines & 69 & 3.88 & & & & \\
\hline & Podcasts & 28 & 1.57 & & & & \\
\hline & Religious Leaders & 4 & 0.22 & & & & \\
\hline & Social Media & 237 & 13.31 & & & & \\
\hline & TV & 889 & 49.94 & & & & \\
\hline \multirow[t]{2}{*}{ Tested for Covid } & No & 1457 & 81.85 & & & & \\
\hline & Yes & 323 & 18.15 & & & & \\
\hline \multirow[t]{2}{*}{ Friends with Covid } & No & 903 & 50.73 & & & & \\
\hline & Yes & 876 & 49.21 & & & & \\
\hline
\end{tabular}

Table B1: Descriptive Statistics 


\begin{tabular}{|c|c|c|c|c|c|c|c|}
\hline \multirow[b]{2}{*}{ Variable } & & \multicolumn{2}{|c|}{$\begin{array}{c}\text { Control } \\
(n=597)\end{array}$} & \multicolumn{2}{|c|}{$\begin{array}{c}\text { Endorsement } \\
\quad(n=591)\end{array}$} & \multicolumn{2}{|c|}{$\begin{array}{c}\text { Norms } \\
(n=592)\end{array}$} \\
\hline & & $\bar{x}$ & $s$ & $\bar{x}$ & $s$ & $\bar{x}$ & $s$ \\
\hline Age & & 54.85 & 16.26 & 53.91 & 16.09 & 53.93 & 15.40 \\
\hline Follows News & & 3.70 & 1.21 & 3.71 & 1.23 & 3.76 & 1.16 \\
\hline Follows Covid & & 3.77 & 0.93 & 3.84 & 0.94 & 3.75 & 0.93 \\
\hline Covid Threat & & 2.47 & 0.60 & 2.48 & 0.62 & 2.45 & 0.64 \\
\hline Variable & Value & $n$ & $\%$ & $n$ & $\%$ & $n$ & $\%$ \\
\hline \multirow[t]{3}{*}{ Gender } & Female & 354 & 59.3 & 364 & 61.6 & 348 & 58.8 \\
\hline & Male & 242 & 40.5 & 227 & 38.4 & 244 & 41.2 \\
\hline & Non-binary & 1 & 0.2 & 0 & 0.0 & 0 & 0.0 \\
\hline \multirow[t]{11}{*}{ Education } & $<$ 8thGrade & 1 & 0.2 & 0 & 0.0 & 0 & 0.0 \\
\hline & $<$ High School & 4 & 0.7 & 5 & 0.8 & 15 & 2.5 \\
\hline & High School & 95 & 15.9 & 79 & 13.4 & 69 & 11.7 \\
\hline & Some College & 108 & 18.1 & 108 & 18.3 & 118 & 19.9 \\
\hline & Vocational School & 23 & 3.9 & 26 & 4.4 & 16 & 2.7 \\
\hline & Associate's & 64 & 10.7 & 86 & 14.6 & 73 & 12.3 \\
\hline & Bachelor's & 161 & 27.0 & 139 & 23.5 & 148 & 25.0 \\
\hline & Some Graduate School & 22 & 3.7 & 16 & 2.7 & 22 & 3.7 \\
\hline & Master's & 101 & 16.9 & 111 & 18.8 & 109 & 18.4 \\
\hline & Professional Degree & 7 & 1.2 & 12 & 2.0 & 13 & 2.2 \\
\hline & Doctorate & 11 & 1.8 & 9 & 1.5 & 9 & 1.5 \\
\hline \multirow[t]{7}{*}{ Party ID } & Strong Democrat & 28 & 4.7 & 37 & 6.3 & 30 & 5.1 \\
\hline & Democrat & 32 & 5.4 & 41 & 6.9 & 37 & 6.2 \\
\hline & Lean Democrat & 23 & 3.9 & 29 & 4.9 & 25 & 4.2 \\
\hline & Independent & 110 & 18.4 & 95 & 16.1 & 89 & 15.0 \\
\hline & Lean Republican & 80 & 13.4 & 78 & 13.2 & 89 & 15.0 \\
\hline & Republican & 139 & 23.3 & 115 & 19.5 & 153 & 25.8 \\
\hline & Strong Republican & 185 & 31.0 & 196 & 33.2 & 169 & 28.5 \\
\hline \multirow[t]{10}{*}{ News Source } & CommunityLeader & 3 & 0.5 & 2 & 0.3 & 3 & 0.5 \\
\hline & Discussion Boards and Aggregators & 3 & 0.5 & 5 & 0.8 & 7 & 1.2 \\
\hline & Friends or Family & 25 & 4.2 & 30 & 5.1 & 23 & 3.9 \\
\hline & Does Not Follow News & 14 & 2.3 & 10 & 1.7 & 18 & 3.0 \\
\hline & Internet News Sites & 150 & 25.1 & 139 & 23.5 & 165 & 27.9 \\
\hline & Newspapers or Magazines & 20 & 3.4 & 29 & 4.9 & 29 & 4.9 \\
\hline & Podcasts & 9 & 1.5 & 7 & 1.2 & 9 & 1.5 \\
\hline & Religious Leaders & 1 & 0.2 & 4 & 0.7 & 3 & 0.5 \\
\hline & Social Media & 83 & 13.9 & 75 & 12.7 & 77 & 13.0 \\
\hline & TV & 289 & 48.4 & 290 & 49.1 & 258 & 43.6 \\
\hline \multirow[t]{10}{*}{ Covid News Source } & Community Leader & 2 & 0.3 & 4 & 0.7 & 7 & 1.2 \\
\hline & Discussion Boards and Aggregators & 3 & 0.5 & 4 & 0.7 & 4 & 0.7 \\
\hline & Friends or Family & 20 & 3.4 & 17 & 2.9 & 20 & 3.4 \\
\hline & Does Not Follow News & 10 & 1.7 & 10 & 1.7 & 10 & 1.7 \\
\hline & Internet News Sites & 146 & 24.5 & 148 & 25.0 & 147 & 24.8 \\
\hline & Newspapers or Magazines & 16 & 2.7 & 25 & 4.2 & 28 & 4.7 \\
\hline & Podcasts & 12 & 2.0 & 7 & 1.2 & 9 & 1.5 \\
\hline & Religious Leaders & 1 & 0.2 & 1 & 0.2 & 2 & 0.3 \\
\hline & Social Media & 90 & 15.1 & 71 & 12.0 & 76 & 12.8 \\
\hline & TV & 297 & 49.7 & 304 & 51.4 & 288 & 48.6 \\
\hline \multirow[t]{2}{*}{ Tested for Covid } & No & 487 & 81.6 & 487 & 82.4 & 483 & 81.6 \\
\hline & Yes & 110 & 18.4 & 104 & 17.6 & 109 & 18.4 \\
\hline \multirow[t]{2}{*}{ Friends with Covid } & No & 302 & 50.6 & 303 & 51.3 & 298 & 50.3 \\
\hline & Yes & 295 & 49.4 & 287 & 48.6 & 294 & 49.7 \\
\hline
\end{tabular}

Table B2: Covariate Balance 


\begin{tabular}{lrrrrrr}
\hline Variable & $n$ & $\bar{x}$ & $s$ & Min & Mdn & Max \\
\hline Religiosity Index & & & & & & \\
Regularly Attends Church & 1777 & 4.46 & 1.69 & 1.00 & 5.00 & 6.00 \\
Spiritual Values & 1778 & 5.28 & 1.03 & 1.00 & 6.00 & 6.00 \\
Americans Should be More Religious & 1779 & 5.04 & 1.16 & 1.00 & 5.00 & 6.00 \\
Religiosity Index (Additive Index) & 1776 & 14.78 & 3.15 & 3.00 & 15.00 & 18.00 \\
Religiosity (Self-Reported) & & & & & & \\
Religiosity & 1780 & 4.31 & 0.72 & 1.00 & 4.00 & 5.00 \\
Religious Motivation & & & & & & \\
Insurance & 1693 & 0.09 & 0.28 & 0.00 & 0.00 & 1.00 \\
Morality & 1693 & 0.13 & 0.34 & 0.00 & 0.00 & 1.00 \\
Social Capital & 1693 & 0.08 & 0.27 & 0.00 & 0.00 & 1.00 \\
Heuristics & 1693 & 0.17 & 0.38 & 0.00 & 0.00 & 1.00 \\
Tradition & 1693 & 0.12 & 0.32 & 0.00 & 0.00 & 1.00 \\
Social Identity & 1693 & 0.41 & 0.49 & 0.00 & 0.00 & 1.00 \\
Extrinsic/Intrinsic Motivation & & & & & & \\
Religion Offers Comfort & 1773 & 4.16 & 0.90 & 1.00 & 4.00 & 5.00 \\
Takes Religious Approach to Life & 1775 & 4.10 & 0.92 & 1.00 & 4.00 & 5.00 \\
Enjoys Social Aspects of Church & 1775 & 2.71 & 1.12 & 1.00 & 3.00 & 5.00 \\
\hline
\end{tabular}

Table B3: Descriptive Statistics (Religious Variables)

\begin{tabular}{|c|c|c|c|c|c|c|}
\hline \multirow[b]{2}{*}{ Variable } & \multicolumn{2}{|c|}{$\begin{array}{c}\text { Control } \\
(n=597)\end{array}$} & \multicolumn{2}{|c|}{$\begin{array}{l}\text { Endorsement } \\
\quad(n=591)\end{array}$} & \multicolumn{2}{|c|}{$\begin{array}{c}\text { Norms } \\
(n=592)\end{array}$} \\
\hline & $\bar{x}$ & $s$ & $\bar{x}$ & $s$ & $\bar{x}$ & $s$ \\
\hline \multicolumn{7}{|l|}{ Religiosity Index } \\
\hline Regularly Attends Church & 4.48 & 1.71 & 4.35 & 1.72 & 4.53 & 1.63 \\
\hline Spiritual Values & 5.20 & 1.11 & 5.27 & 1.03 & 5.38 & 0.91 \\
\hline Americans Should be More Religious & 5.02 & 1.15 & 5.01 & 1.22 & 5.11 & 1.10 \\
\hline Religiosity Index (Additive Index) & 14.70 & 3.29 & 14.62 & 3.24 & 15.02 & 2.89 \\
\hline \multicolumn{7}{|l|}{ Religiosity (Self-Reported) } \\
\hline Religiosity & 4.34 & 0.72 & 4.28 & 0.73 & 4.31 & 0.71 \\
\hline \multicolumn{7}{|l|}{ Religious Motivation } \\
\hline Insurance & 0.09 & 0.29 & 0.08 & 0.28 & 0.08 & 0.28 \\
\hline Morality & 0.13 & 0.34 & 0.12 & 0.32 & 0.15 & 0.35 \\
\hline Social Capital & 0.08 & 0.28 & 0.09 & 0.29 & 0.06 & 0.24 \\
\hline Heuristics & 0.14 & 0.35 & 0.21 & 0.40 & 0.17 & 0.37 \\
\hline Tradition & 0.12 & 0.32 & 0.12 & 0.33 & 0.12 & 0.32 \\
\hline Social Identity & 0.43 & 0.50 & 0.38 & 0.49 & 0.42 & 0.49 \\
\hline \multicolumn{7}{|l|}{ Extrinsic/Intrinsic Motivation } \\
\hline Religion Offers Comfort & 4.17 & 0.87 & 4.17 & 0.89 & 4.13 & 0.93 \\
\hline Takes Religious Approach to Life & 4.09 & 0.95 & 4.06 & 0.92 & 4.14 & 0.89 \\
\hline Enjoys Social Aspects of Church & 2.70 & 1.15 & 2.71 & 1.11 & 2.73 & 1.11 \\
\hline
\end{tabular}

Table B4: Balance (Religious Variables) 


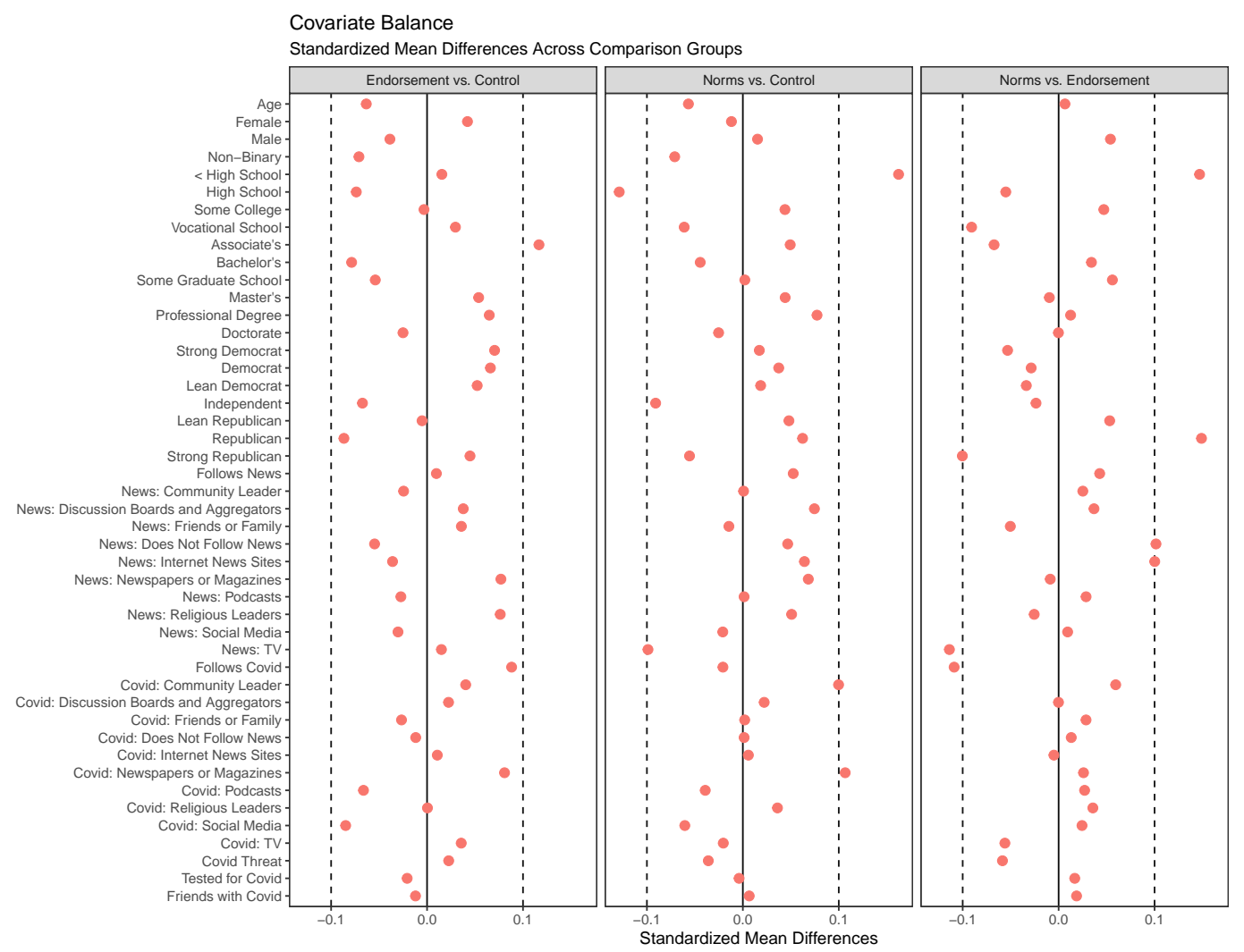

Figure B3: Covariate Balance 


\section{Correlates of Mask Wearing}

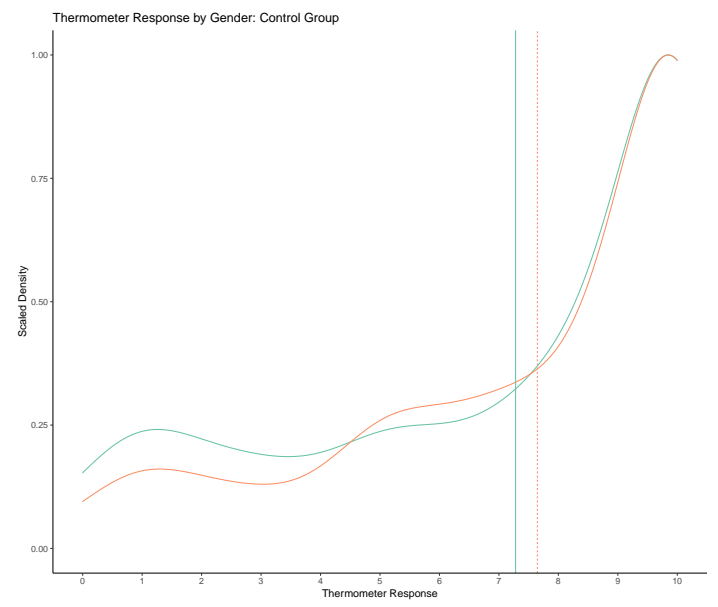

(a) Mask wearing by gender

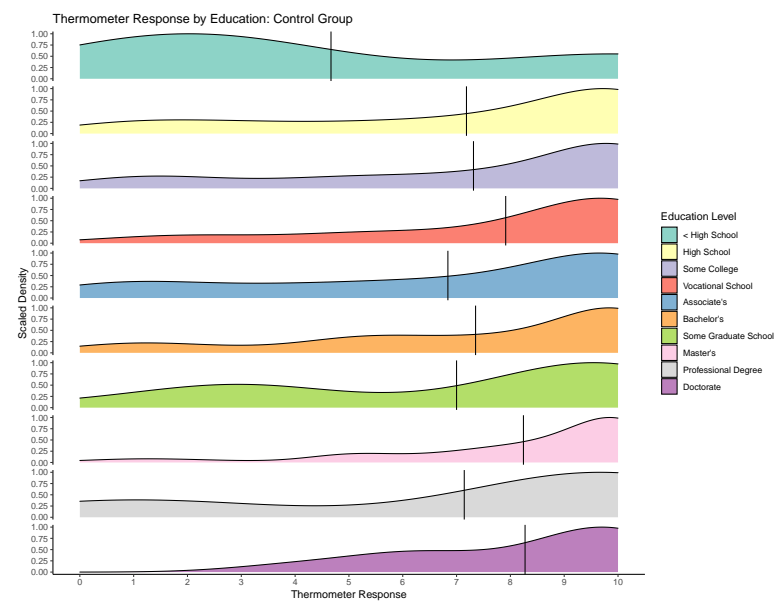

(c) Mask wearing by education

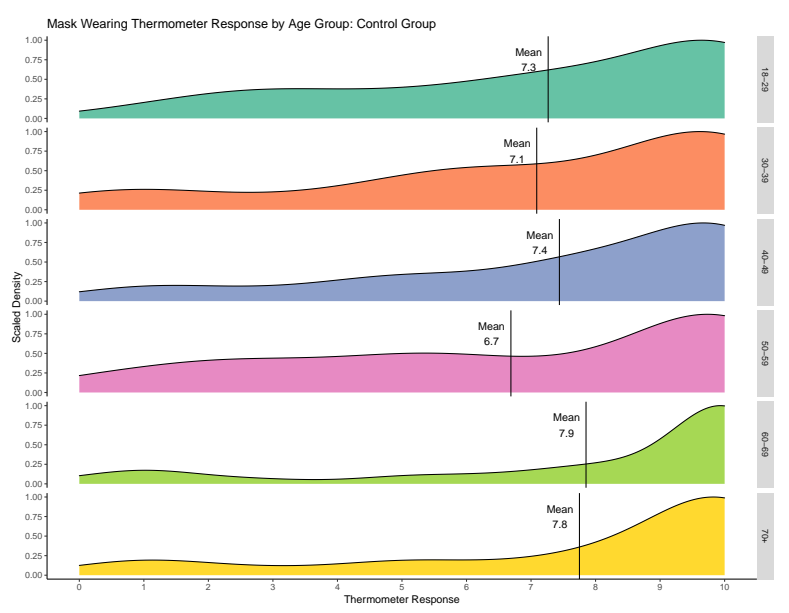

(b) Mask wearing by age

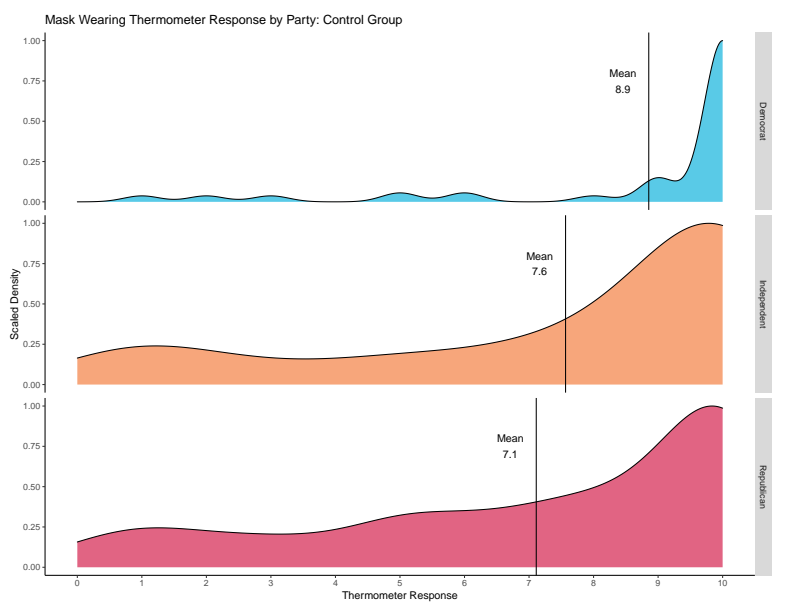

(d) Mask wearing by party ID

Figure C4: Who wears a mask? Raw Distributions 


\begin{tabular}{lcc}
\hline & DV: Thermometer \\
\hline Intercept & -2.456 & $(1.745)$ \\
Male & 0.078 & $(0.237)$ \\
Age 30-39 & $-1.071^{* *}$ & $(0.506)$ \\
Age 40-49 & $-0.891^{*}$ & $(0.483)$ \\
Age 50-59 & -0.761 & $(0.469)$ \\
Age 60-69 & 0.109 & $(0.443)$ \\
Age 70+ & 0.131 & $(0.459)$ \\
High School & 1.845 & $(1.529)$ \\
Some College & 2.279 & $(1.527)$ \\
Vocational School & $2.836^{*}$ & $(1.600)$ \\
Associate's & 2.232 & $(1.540)$ \\
Bachelor's & $2.558^{*}$ & $(1.521)$ \\
Some Graduate School & 2.009 & $(1.611)$ \\
Master's & $3.063^{* *}$ & $(1.539)$ \\
Professional Degree & 2.385 & $(1.804)$ \\
Doctorate & 2.209 & $(1.712)$ \\
Democrat & 0.485 & $(0.698)$ \\
Lean Democrat & 0.491 & $(0.793)$ \\
Independent & 0.117 & $(0.587)$ \\
Lean Republican & 0.438 & $(0.605)$ \\
Republican & 0.257 & $(0.581)$ \\
Strong Republican & -0.320 & $(0.572)$ \\
Covid Threat & $2.514^{* * *}$ & $(0.211)$ \\
Tested for Covid & 0.149 & $(0.302)$ \\
Friends with Covid & -0.049 & $(0.227)$ \\
\hline Observations & 566 & \\
$R^{2}$ & 0.367 & \\
$F$ & 12.037 & \\
$*$ * $<$ 0.1, ** $p<0.05, * * *$ & $p<0.01$ \\
Note: Standard errors in & parentheses. \\
\hline & & \\
\hline
\end{tabular}

Table C5: Demographic Correlates of Mask Wearing (Control Condition) 


\begin{tabular}{|c|c|c|c|c|c|}
\hline & \multicolumn{5}{|c|}{ DV: Thermometer } \\
\hline & $(1)$ & $(2)$ & $(3)$ & $(4)$ & $(5)$ \\
\hline Intercept & $\begin{array}{c}5.407^{* * *} \\
(1.206)\end{array}$ & $\begin{array}{c}7.857^{* * *} \\
(0.453)\end{array}$ & $\begin{array}{c}5.700^{* * *} \\
(0.999)\end{array}$ & $\begin{array}{c}6.497 * * * \\
(0.892)\end{array}$ & $\begin{array}{l}3.079^{*} \\
(1.817)\end{array}$ \\
\hline Religion Offers Comfort: Disagree & $\begin{array}{l}-2.514 \\
(1.805)\end{array}$ & & & & $\begin{array}{l}-2.794 \\
(1.942)\end{array}$ \\
\hline Religion Offers Comfort: Neither Agree nor Disagree & $\begin{array}{l}-1.329 \\
(1.742)\end{array}$ & & & & $\begin{array}{l}-1.116 \\
(1.868)\end{array}$ \\
\hline Religion Offers Comfort: Agree & $\begin{array}{l}-1.692 \\
(1.725)\end{array}$ & & & & $\begin{array}{l}-1.502 \\
(1.843)\end{array}$ \\
\hline Religion Offers Comfort: Strongly Agree & $\begin{array}{l}-1.417 \\
(1.714)\end{array}$ & & & & $\begin{array}{l}-1.326 \\
(1.823)\end{array}$ \\
\hline Religious Approach to Life: Disagree & $\begin{array}{c}4.362^{* * *} \\
(1.545)\end{array}$ & & & & $\begin{array}{c}4.482^{* * *} \\
(1.665)\end{array}$ \\
\hline Religious Approach to Life: Neither Agree nor Disagree & $\begin{array}{l}3.086^{* *} \\
(1.483)\end{array}$ & & & & $\begin{array}{l}3.153^{*} \\
(1.656)\end{array}$ \\
\hline Religious Approach to Life: Agree & $\begin{array}{l}3.393^{* *} \\
(1.459)\end{array}$ & & & & $\begin{array}{c}3.583^{* *} \\
(1.628)\end{array}$ \\
\hline Religious Approach to Life: Strongly Agree & $\begin{array}{l}2.817^{*} \\
(1.445)\end{array}$ & & & & $\begin{array}{l}3.252^{* *} \\
(1.632)\end{array}$ \\
\hline Enjoys Social Aspects of Church: Disagree & $\begin{array}{c}0.666 \\
(0.420)\end{array}$ & & & & $\begin{array}{l}0.506 \\
(0.444)\end{array}$ \\
\hline Enjoys Social Aspects of Church: Neither Agree nor Disagree & $\begin{array}{c}0.273 \\
(0.421)\end{array}$ & & & & $\begin{array}{l}0.050 \\
(0.451)\end{array}$ \\
\hline Enjoys Social Aspects of Church: Agree & $\begin{array}{c}0.396 \\
(0.480)\end{array}$ & & & & $\begin{array}{l}-0.054 \\
(0.544)\end{array}$ \\
\hline Enjoys Social Aspects of Church: Strongly Agree & $\begin{array}{l}1.432^{* *} \\
(0.614)\end{array}$ & & & & $\begin{array}{l}1.093 \\
(0.684)\end{array}$ \\
\hline Morality & & $\begin{array}{c}0.129 \\
(0.589)\end{array}$ & & & $\begin{array}{c}0.056 \\
(0.602)\end{array}$ \\
\hline Social Capital & & $\begin{array}{l}-0.346 \\
(0.663)\end{array}$ & & & $\begin{array}{c}0.027 \\
(0.685)\end{array}$ \\
\hline Heuristics & & $\begin{array}{l}-0.515 \\
(0.586)\end{array}$ & & & $\begin{array}{l}-0.438 \\
(0.608)\end{array}$ \\
\hline Tradition & & $\begin{array}{l}-0.111 \\
(0.596)\end{array}$ & & & $\begin{array}{l}-0.395 \\
(0.615)\end{array}$ \\
\hline Social Identity & & $\begin{array}{l}-0.758 \\
(0.497)\end{array}$ & & & $\begin{array}{l}-0.744 \\
(0.526)\end{array}$ \\
\hline Religiosity (SR) Slightly Religious & & & $\begin{array}{l}1.550 \\
(1.084)\end{array}$ & & $\begin{array}{l}1.344 \\
(1.192)\end{array}$ \\
\hline Religiosity (SR) Moderately Religious & & & $\begin{array}{l}1.950^{*} \\
(1.020)\end{array}$ & & $\begin{array}{l}2.153^{*} \\
(1.156)\end{array}$ \\
\hline Religiosity (SR) Very Religious & & & $\begin{array}{c}1.637 \\
(1.017)\end{array}$ & & $\begin{array}{l}2.039^{*} \\
(1.163)\end{array}$ \\
\hline Regularly Attends Church: Strongly Agree & & & & $\begin{array}{l}1.033^{*} \\
(0.600)\end{array}$ & $\begin{array}{c}0.659 \\
(0.666)\end{array}$ \\
\hline Regularly Attends Church: Disagree & & & & $\begin{array}{c}0.485 \\
(0.670)\end{array}$ & $\begin{array}{c}0.019 \\
(0.727)\end{array}$ \\
\hline Regularly Attends Church: Somewhat Disagree & & & & $\begin{array}{l}1.544^{* *} \\
(0.688)\end{array}$ & $\begin{array}{c}0.992 \\
(0.746)\end{array}$ \\
\hline Regularly Attends Church: Somewhat Agree & & & & $\begin{array}{c}0.724 \\
(0.670)\end{array}$ & $\begin{array}{c}0.395 \\
(0.745)\end{array}$ \\
\hline Regularly Attends Church: Agree & & & & $\begin{array}{c}0.281 \\
(0.644)\end{array}$ & $\begin{array}{c}-0.076 \\
(0.718)\end{array}$ \\
\hline Spiritual Values: Strongly Agree & & & & $\begin{array}{c}0.188 \\
(1.357)\end{array}$ & $\begin{array}{c}0.753 \\
(1.558)\end{array}$ \\
\hline Spiritual Values: Disagree & & & & $\begin{array}{c}0.980 \\
(2.046)\end{array}$ & $\begin{array}{c}1.827 \\
(2.253)\end{array}$ \\
\hline Spiritual Values: Somewhat Disagree & & & & $\begin{array}{c}1.375 \\
(1.531)\end{array}$ & $\begin{array}{c}1.778 \\
(1.750)\end{array}$ \\
\hline Spiritual Values: Somewhat Agree & & & & $\begin{array}{c}1.076 \\
(1.356)\end{array}$ & $\begin{array}{c}1.456 \\
(1.558)\end{array}$ \\
\hline Spiritual Values: Agree & & & & $\begin{array}{c}0.936 \\
(1.361)\end{array}$ & $\begin{array}{c}1.235 \\
(1.571)\end{array}$ \\
\hline Americans Should be More Religious: Strongly Agree & & & & $\begin{array}{l}-0.422 \\
(1.302)\end{array}$ & $\begin{array}{l}-0.966 \\
(1.487)\end{array}$ \\
\hline Americans Should be More Religious: Disagree & & & & $\begin{array}{l}-0.073 \\
(2.008)\end{array}$ & $\begin{array}{c}0.786 \\
(2.171)\end{array}$ \\
\hline Americans Should be More Religious: Somewhat Disagree & & & & $\begin{array}{c}-0.428 \\
(1.398)\end{array}$ & $\begin{array}{l}-0.325 \\
(1.662)\end{array}$ \\
\hline Americans Should be More Religious: Somewhat Agree & & & & $\begin{array}{l}-0.416 \\
(1.296)\end{array}$ & $\begin{array}{c}-0.794 \\
(1.490)\end{array}$ \\
\hline Americans Should be More Religious: Agree & & & & $\begin{array}{c}-0.403 \\
(1.307)\end{array}$ & $\begin{array}{c}-0.818 \\
(1.493)\end{array}$ \\
\hline Observations & 564 & 544 & 567 & 566 & 540 \\
\hline$R^{2}$ & 0.043 & 0.012 & 0.008 & 0.029 & 0.084 \\
\hline$F$ & 2.073 & 1.286 & 1.520 & 1.087 & 1.327 \\
\hline
\end{tabular}

Table C6: Religious Correlates of Mask Wearing (Control Condition) 


\section{OLS Analyses: Average Treatment Effects}

\begin{tabular}{lccc}
\hline & \multicolumn{3}{c}{ DV: Thermometer } \\
\cline { 2 - 4 } & $(1)$ & $(2)$ & $(3)$ \\
\hline Intercept & $7.430^{* * *}$ & 0.403 & 0.529 \\
& $(0.126)$ & $(0.500)$ & $(1.127)$ \\
Endorsement & $0.442^{* *}$ & $0.423^{* * *}$ & $0.340^{* *}$ \\
& $(0.178)$ & $(0.151)$ & $(0.147)$ \\
Norms & $0.427^{* *}$ & $0.450^{* * *}$ & $0.452^{* * *}$ \\
& $(0.179)$ & $(0.151)$ & $(0.148)$ \\
\hline Controls & No & Demographic & All \\
Observations & 1685 & 1683 & 1682 \\
$R^{2}$ & 0.005 & 0.297 & 0.344 \\
$F$ & 3.977 & 50.243 & 25.390 \\
\hline${ }^{*} p<0.1,{ }^{* *} p<0.05,{ }^{* * *} p<0.01$ & \\
\hline
\end{tabular}

Table D7: Thermometer

\begin{tabular}{lccc}
\hline & \multicolumn{3}{c}{ DV: Hand Washing } \\
\cline { 2 - 4 } & $(1)$ & $(2)$ & $(3)$ \\
\hline Intercept & $4.480^{* * *}$ & $4.005^{* * *}$ & $3.568^{* * *}$ \\
& $(0.030)$ & $(0.137)$ & $(0.309)$ \\
Endorsement & 0.046 & 0.027 & 0.018 \\
& $(0.042)$ & $(0.042)$ & $(0.041)$ \\
Norms & 0.061 & 0.068 & 0.065 \\
& $(0.042)$ & $(0.041)$ & $(0.041)$ \\
\hline Controls & No & Demographic & All \\
Observations & 1779 & 1776 & 1774 \\
$R^{2}$ & 0.001 & 0.059 & 0.095 \\
$F$ & 1.119 & 7.324 & 5.234 \\
\hline${ }^{*} p<0.1,{ }^{* *} p<0.05, * * * p<0.01$ & \\
\hline
\end{tabular}

Table D8: Policy: Hand Washing 


\begin{tabular}{|c|c|c|c|}
\hline & \multicolumn{3}{|c|}{ DV: Distancing } \\
\hline & (1) & $(2)$ & (3) \\
\hline & Model1 & Model2 & Model3 \\
\hline Intercept & $\begin{array}{c}4.310^{* * *} \\
(0.034)\end{array}$ & $\begin{array}{c}2.959^{* * *} \\
(0.151)\end{array}$ & $\begin{array}{c}3.320^{* * *} \\
(0.338)\end{array}$ \\
\hline Endorsement & $\begin{array}{c}0.127^{* * *} \\
(0.049)\end{array}$ & $\begin{array}{c}0.109^{* *} \\
(0.046)\end{array}$ & $\begin{array}{l}0.086^{*} \\
(0.045)\end{array}$ \\
\hline Norms & $\begin{array}{c}0.129^{* * *} \\
(0.049)\end{array}$ & $\begin{array}{c}0.138^{* * *} \\
(0.046)\end{array}$ & $\begin{array}{c}0.137^{* * *} \\
(0.045)\end{array}$ \\
\hline Controls & No & Demographic & All \\
\hline Observations & 1780 & 1777 & 1775 \\
\hline$R^{2}$ & 0.005 & 0.138 & 0.192 \\
\hline$F$ & 4.581 & 18.745 & 11.803 \\
\hline
\end{tabular}

Table D9: Policy: Distancing

\begin{tabular}{lccc}
\hline & \multicolumn{3}{c}{ DV: Mask Wearing } \\
\cline { 2 - 4 } & $(1)$ & $(2)$ & $(3)$ \\
\hline Intercept & $4.072^{* * *}$ & $1.846^{* * *}$ & $1.655^{* * *}$ \\
& $(0.043)$ & $(0.180)$ & $(0.400)$ \\
Endorsement & 0.097 & 0.076 & 0.046 \\
& $(0.062)$ & $(0.054)$ & $(0.053)$ \\
Norms & $0.156^{* *}$ & $0.168^{* * *}$ & $0.165^{* * *}$ \\
& $(0.062)$ & $(0.054)$ & $(0.053)$ \\
\hline Controls & No & Demographic & All \\
Observations & 1780 & 1777 & 1775 \\
$R^{2}$ & 0.004 & 0.234 & 0.283 \\
$F$ & 3.279 & 35.885 & 19.597 \\
\hline${ }^{*} p<0.1,{ }^{* *} p<0.05, * * * p<0.01$ & \\
\hline
\end{tabular}

Table D10: Policy: Mask Wearing 


\begin{tabular}{lccc}
\hline & \multicolumn{3}{c}{ DV: Prayer } \\
\cline { 2 - 4 } & $(1)$ & $(2)$ & $(3)$ \\
\hline Intercept & $3.616^{* * *}$ & $2.778^{* * *}$ & $2.248^{* * *}$ \\
& $(0.049)$ & $(0.226)$ & $(0.514)$ \\
Endorsement & 0.078 & 0.079 & 0.085 \\
& $(0.070)$ & $(0.069)$ & $(0.068)$ \\
Norms & 0.038 & 0.055 & 0.073 \\
& $(0.070)$ & $(0.068)$ & $(0.068)$ \\
\hline Controls & No & Demographic & All \\
Observations & 1779 & 1776 & 1774 \\
$R^{2}$ & 0.001 & 0.058 & 0.081 \\
$F$ & 0.620 & 7.191 & 4.384 \\
\hline${ }^{*} p<0.1,{ }^{* *} p<0.05,{ }^{* * *} p<0.01$ & \\
\hline
\end{tabular}

Table D11: Policy: Prayer

\begin{tabular}{lccc}
\hline & \multicolumn{3}{c}{ DV: Would Click } \\
\cline { 2 - 4 } & $(1)$ & $(2)$ & $(3)$ \\
\hline Intercept & $6.096^{* * *}$ & -1.020 & -1.748 \\
& $(0.155)$ & $(0.625)$ & $(1.371)$ \\
Endorsement & 0.353 & 0.308 & 0.186 \\
& $(0.219)$ & $(0.189)$ & $(0.184)$ \\
Norms & 0.286 & $0.338^{*}$ & $0.341^{*}$ \\
& $(0.220)$ & $(0.189)$ & $(0.184)$ \\
\hline Controls & No & Demographic & All \\
Observations & 1743 & 1740 & 1739 \\
$R^{2}$ & 0.002 & 0.273 & 0.327 \\
$F$ & 1.463 & 43.188 & 23.590 \\
\hline${ }^{*} p<0.1,{ }^{* *} p<0.05,{ }^{* * *} p<0.01$ & \\
\hline
\end{tabular}

Table D12: Would Click

\begin{tabular}{lccc}
\hline & \multicolumn{3}{c}{ DV: Clicked } \\
\cline { 2 - 4 } & $(1)$ & $(2)$ & $(3)$ \\
\hline Intercept & $0.138^{* * *}$ & 0.062 & 0.030 \\
& $(0.017)$ & $(0.083)$ & $(0.160)$ \\
Endorsement & -0.026 & -0.027 & -0.029 \\
& $(0.024)$ & $(0.024)$ & $(0.024)$ \\
Norms & 0.016 & 0.018 & 0.020 \\
& $(0.024)$ & $(0.024)$ & $(0.024)$ \\
\hline Controls & No & Demographic & All \\
Observations & 1216 & 1214 & 1213 \\
$R^{2}$ & 0.003 & 0.024 & 0.049 \\
$F$ & 1.593 & 1.961 & 1.729 \\
\hline${ }^{*} p<0.1,{ }^{* *} p<0.05,{ }^{* * *} p<0.01$ \\
\hline
\end{tabular}

Table D13: Clicked 


\section{E Compliance Analyses: Compliance Average Causal Effects}

\begin{tabular}{lcccccc}
\hline & \multicolumn{5}{c}{ DV: Thermometer } \\
\cline { 2 - 6 } & $(1)$ & $(2)$ & $(3)$ & $(4)$ & $(5)$ & $(6)$ \\
\hline Intercept & $7.430^{* * *}$ & $7.331^{* * *}$ & $7.309^{* * *}$ & $7.430^{* * *}$ & $7.378^{* * *}$ & $7.377^{* * *}$ \\
& $(0.128)$ & $(0.127)$ & $(0.127)$ & $(0.126)$ & $(0.126)$ & $(0.126)$ \\
Endorsement (Compliant) & $0.460^{* *}$ & $0.672^{* * *}$ & $0.716^{* * *}$ & & & \\
Norms (Compliant) & $(0.188)$ & $(0.188)$ & $(0.188)$ & & & \\
& & & & $0.461^{* *}$ & $0.573^{* * *}$ & $0.573^{* * *}$ \\
& & & & $(0.193)$ & $(0.193)$ & $(0.193)$ \\
\hline Controls & No & Demographic & All & No & Demographic & All \\
Observations & 1130 & 1129 & 1128 & 1122 & 1121 & 1120 \\
$R^{2}$ & 0.017 & 0.021 & 0.021 & 0.012 & 0.013 & 0.013 \\
Adjusted $R^{2}$ & 0.016 & 0.020 & 0.020 & 0.011 & 0.012 & 0.012 \\
\hline${ }^{*} p<0.1,{ }^{* *} p<0.05,{ }^{* * *} p<0.01$ & & & & \\
\hline
\end{tabular}

Table E14: Thermometer

\begin{tabular}{lcccccc}
\hline & \multicolumn{5}{c}{ DV: Hand washing } \\
\cline { 2 - 7 } & $(1)$ & $(2)$ & $(3)$ & $(4)$ & $(5)$ & $(6)$ \\
\hline Intercept & $4.480^{* * *}$ & $4.473^{* * *}$ & $4.471^{* * *}$ & $4.480^{* * *}$ & $4.476^{* * *}$ & $4.477^{* * *}$ \\
& $(0.031)$ & $(0.031)$ & $(0.031)$ & $(0.029)$ & $(0.029)$ & $(0.029)$ \\
Endorsement (Compliant) & 0.048 & 0.061 & 0.065 & & & \\
Norms (Compliant) & $(0.046)$ & $(0.046)$ & $(0.046)$ & & & $0.073^{*}$ \\
& & & & 0.066 & 0.072 \\
Controls & & & & $(0.044)$ & $(0.044)$ & $(0.044)$ \\
Observations & No & Demographic & All & No & Demographic & All \\
$R^{2}$ & 1187 & 1185 & 1184 & 1188 & 1187 & 1185 \\
Adjusted $R^{2}$ & 0.002 & 0.002 & 0.002 & 0.004 & 0.004 & 0.004 \\
\hline${ }^{*} p<0.1,{ }^{* *} p<0.05,{ }^{* * *} p<0.01$ & 0.001 & 0.001 & 0.001 & 0.003 & 0.003 & 0.003 \\
\hline
\end{tabular}

Table E15: Policy: Hand Washing 


\begin{tabular}{lcccccc}
\hline & \multicolumn{5}{c}{ DV: Distancing } \\
\cline { 2 - 7 } & $(1)$ & $(2)$ & $(3)$ & $(4)$ & $(5)$ & $(6)$ \\
\hline Intercept & $4.310^{* * *}$ & $4.290^{* * *}$ & $4.283^{* * *}$ & $4.310^{* * *}$ & $4.299^{* * *}$ & $4.296^{* * *}$ \\
& $(0.036)$ & $(0.036)$ & $(0.036)$ & $(0.035)$ & $(0.035)$ & $(0.035)$ \\
Endorsement (Compliant) & $0.132^{* *}$ & $0.173^{* * *}$ & $0.189^{* * *}$ & & & \\
Norms (Compliant) & $(0.053)$ & $(0.053)$ & $(0.053)$ & & & \\
& & & & $0.140^{* * *}$ & $0.163^{* * *}$ & $0.169^{* * *}$ \\
& & & & $(0.053)$ & $(0.053)$ & $(0.053)$ \\
\hline Controls & No & Demographic & All & No & Demographic & All \\
Observations & 1188 & 1186 & 1185 & 1189 & 1188 & 1186 \\
$R^{2}$ & 0.015 & 0.017 & 0.017 & 0.010 & 0.011 & 0.010 \\
Adjusted $R^{2}$ & 0.014 & 0.016 & 0.016 & 0.009 & 0.010 & 0.010 \\
\hline$* p<0.1, * * p<0.05, * * * p<0.01$ & & & & & \\
\hline
\end{tabular}

Table E16: Policy: Distancing

\begin{tabular}{lcccccc}
\hline & \multicolumn{5}{c}{ DV: Mask wearing } \\
\cline { 2 - 6 } & Model1 & Model2 & Model3 & Model4 & Model5 & Model6 \\
\hline Intercept & $4.072^{* * *}$ & $4.039^{* * *}$ & $4.031^{* * *}$ & $4.072^{* * *}$ & $4.053^{* * *}$ & $4.052^{* * *}$ \\
& $(0.045)$ & $(0.045)$ & $(0.045)$ & $(0.042)$ & $(0.042)$ & $(0.042)$ \\
Endorsement (Compliant) & 0.101 & $0.168^{* *}$ & $0.185^{* * *}$ & & & \\
& $(0.066)$ & $(0.066)$ & $(0.066)$ & & & \\
Norms (Compliant) & & & & $0.169^{* * *}$ & $0.209^{* * *}$ & $0.212^{* * *}$ \\
& & & & $(0.065)$ & $(0.065)$ & $(0.065)$ \\
\hline Controls & No & Demographic & All & No & Demographic & All \\
Observations & 1188 & 1186 & 1185 & 1189 & 1188 & 1186 \\
$R^{2}$ & 0.009 & 0.013 & 0.013 & 0.014 & 0.016 & 0.015 \\
Adjusted $R^{2}$ & 0.008 & 0.012 & 0.012 & 0.013 & 0.015 & 0.015 \\
\hline${ }^{*} p<0.1,{ }^{* *} p<0.05,{ }^{* * *} p<0.01$ & & & & & \\
\hline
\end{tabular}

Table E17: Policy: Mask Wearing 


\begin{tabular}{lcccccc}
\hline & \multicolumn{5}{c}{ DV: Prayer } \\
\cline { 2 - 7 } & $(1)$ & $(2)$ & $(3)$ & $(4)$ & $(5)$ & $(6)$ \\
\hline Intercept & $3.616^{* * *}$ & $3.612^{* * *}$ & $3.614^{* * *}$ & $3.616^{* * *}$ & $3.611^{* * *}$ & $3.614^{* * *}$ \\
& $(0.050)$ & $(0.050)$ & $(0.050)$ & $(0.049)$ & $(0.049)$ & $(0.049)$ \\
Endorsement (Compliant) & 0.081 & 0.087 & 0.086 & & & \\
& $(0.073)$ & $(0.073)$ & $(0.073)$ & & & 0.049 \\
Norms (Compliant) & & & & 0.041 & 0.047 \\
& & & & $(0.076)$ & $(0.076)$ & $(0.075)$ \\
\hline Controls & No & Demographic & All & No & Demographic & All \\
Observations & 1187 & 1185 & 1184 & 1188 & 1187 & 1185 \\
$R^{2}$ & 0.001 & 0.001 & 0.001 & 0.001 & 0.002 & 0.002 \\
Adjusted $R^{2}$ & 0.000 & 0.000 & 0.000 & 0.001 & 0.001 & 0.001 \\
\hline${ }^{*} p<0.1,{ }^{* *} p<0.05,{ }^{* * *} p<0.01$ & & & & & \\
\hline
\end{tabular}

Table E18: Policy: Prayer

\begin{tabular}{lcccccc}
\hline & \multicolumn{5}{c}{ DV: Would click } \\
\cline { 2 - 6 } & $(1)$ & $(2)$ & $(3)$ & $(4)$ & $(5)$ & $(6)$ \\
\hline Intercept & $6.096^{* * *}$ & $5.983^{* * *}$ & $5.959^{* * *}$ & $6.096^{* * *}$ & $6.012^{* * *}$ & $6.005^{* * *}$ \\
& $(0.156)$ & $(0.156)$ & $(0.156)$ & $(0.154)$ & $(0.154)$ & $(0.154)$ \\
Endorsement (Compliant) & 0.368 & $0.616^{* * *}$ & $0.666^{* * *}$ & & & \\
Norms (Compliant) & $(0.230)$ & $(0.229)$ & $(0.229)$ & & & \\
& & & & 0.311 & $0.488^{* *}$ & $0.503^{* *}$ \\
& & & & $(0.237)$ & $(0.237)$ & $(0.236)$ \\
\hline Controls & No & Demographic & All & No & Demographic & All \\
Observations & 1165 & 1163 & 1162 & 1160 & 1159 & 1158 \\
$R^{2}$ & 0.008 & 0.010 & 0.011 & 0.006 & 0.007 & 0.007 \\
Adjusted $R^{2}$ & 0.007 & 0.009 & 0.010 & 0.005 & 0.007 & 0.007 \\
\hline${ }^{*} p<0.1,{ }^{* *} p<0.05,{ }^{* * *} p<0.01$ & & & & & \\
\hline
\end{tabular}

Table E19: Would Click

\begin{tabular}{lcccccc}
\hline & \multicolumn{5}{c}{ DV: Clicked } \\
\cline { 2 - 6 } & $(1)$ & $(2)$ & $(3)$ & $(4)$ & $(5)$ & $(6)$ \\
\hline Intercept & $0.138^{* * *}$ & $0.138^{* * *}$ & $0.136^{* * *}$ & $0.138^{* * *}$ & $0.138^{* * *}$ & $0.136^{* * *}$ \\
& $(0.017)$ & $(0.017)$ & $(0.017)$ & $(0.018)$ & $(0.018)$ & $(0.018)$ \\
Endorsement (Compliant) & -0.026 & -0.027 & -0.024 & & & \\
Norms (Compliant) & $(0.023)$ & $(0.023)$ & $(0.023)$ & & & \\
& & & & 0.016 & 0.017 & 0.018 \\
Controls & & & $(0.026)$ & $(0.026)$ & $(0.026)$ \\
Observations & No & Demographic & All & No & Demographic & All \\
$R^{2}$ & 805 & 804 & 803 & 803 & 802 & 801 \\
Adjusted $R^{2}$ & 0.002 & 0.001 & 0.001 & 0.001 & 0.001 & 0.001 \\
${ }^{*} p<0.1, * * p<0.05, * * * p<0.01$ & 0.000 & 0.000 & 0.000 & 0.000 & 0.000 & 0.000 \\
\hline
\end{tabular}

Table E20: Clicked 


\section{F Mediation Analyses}

\section{F.1 Religiosity (Self-Reported)}

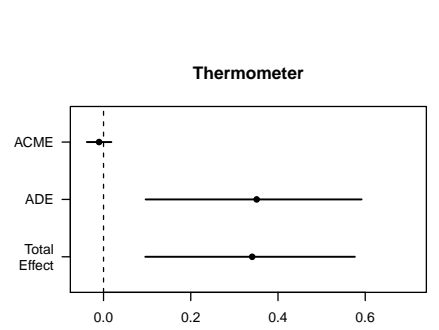

Mediator Variable: Religiosity (Self-Reported) Endorsement Condition
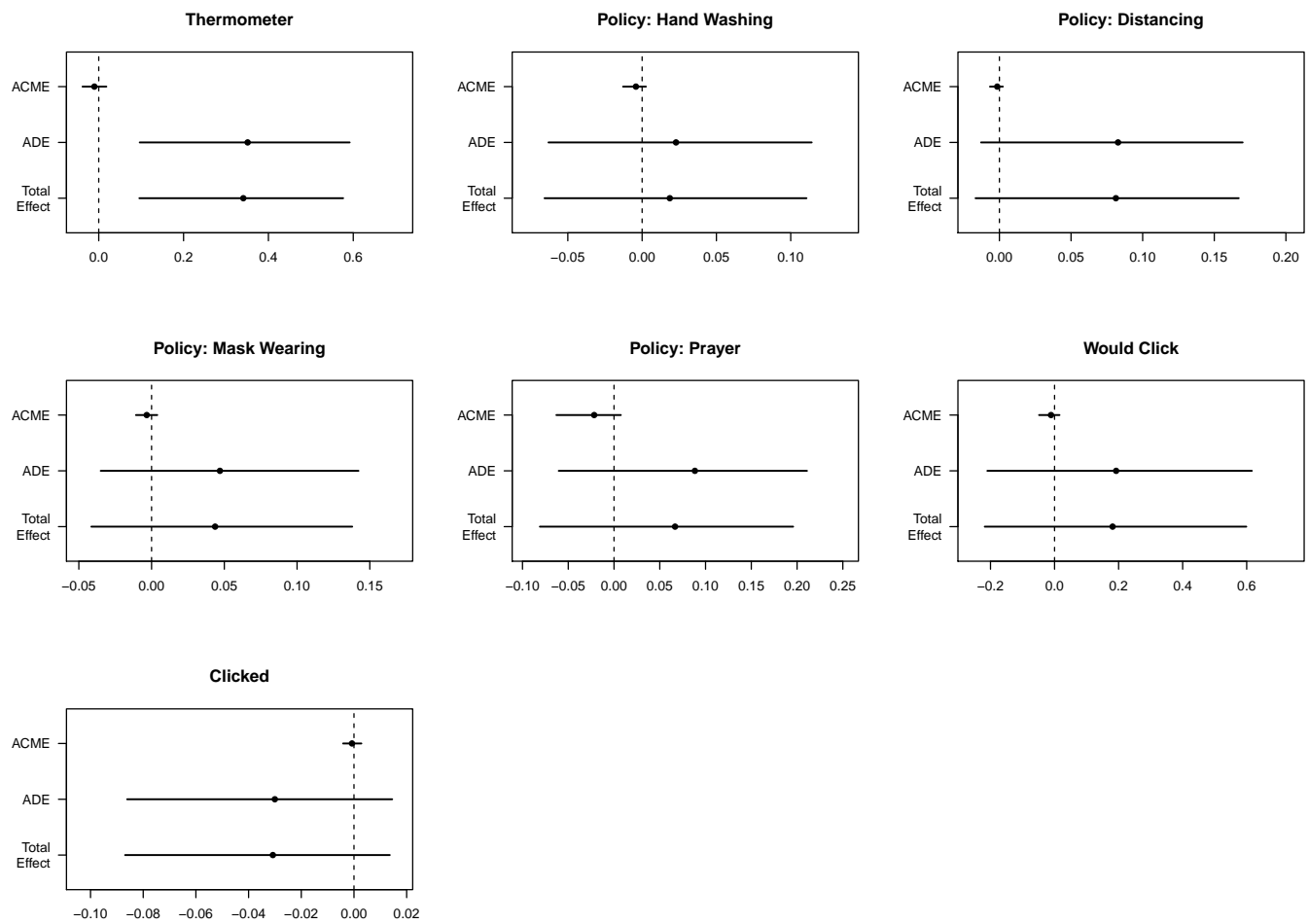


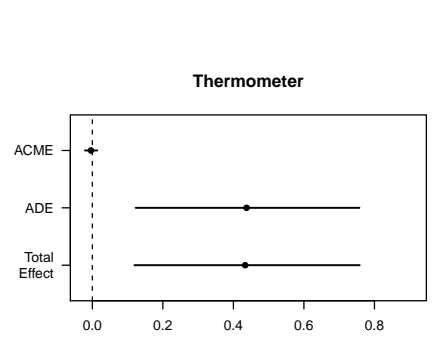

Mediator Variable: Religiosity (Self-Reported) Norms Condition
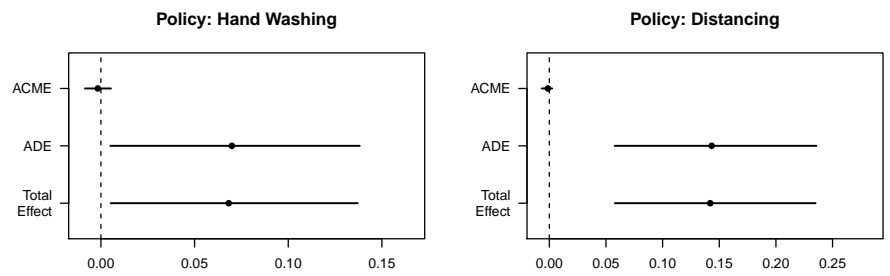

Policy: Mask Wearing

Policy: Prayer

Would Click
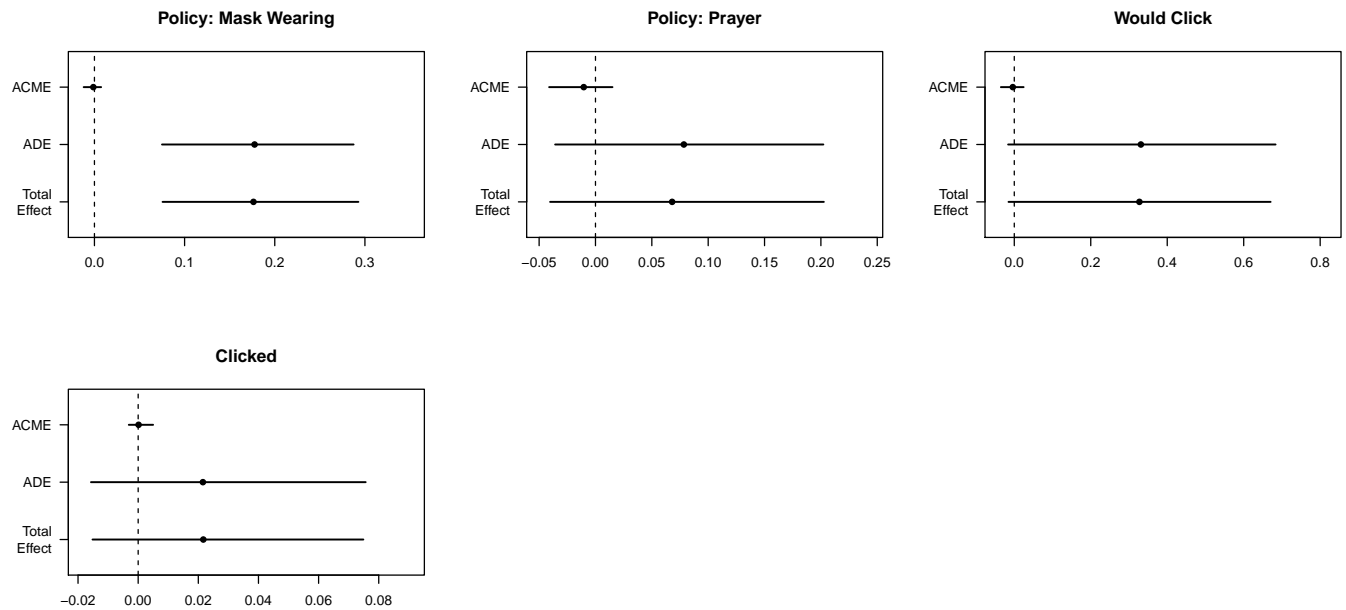


\section{F.2 Religious Motivation}

Table F21: Religious Motivation Variables and Response Wording

\begin{tabular}{ll}
\hline Mediator Variable & Question Wording: My Church is important to me because... \\
\hline Insurance & It helps me in times of personal or financial need. \\
Morality & Following the advice of my Church is the right thing to do. \\
Social Capital & It is where I find my family, close friends, and / or business partners. \\
Heuristics & It makes it easier for me to reach decisions about complicated things. \\
Tradition & I was born into it and / or brought up that way. \\
Social Identity & I identify as an Evangelical Christian. \\
\hline
\end{tabular}
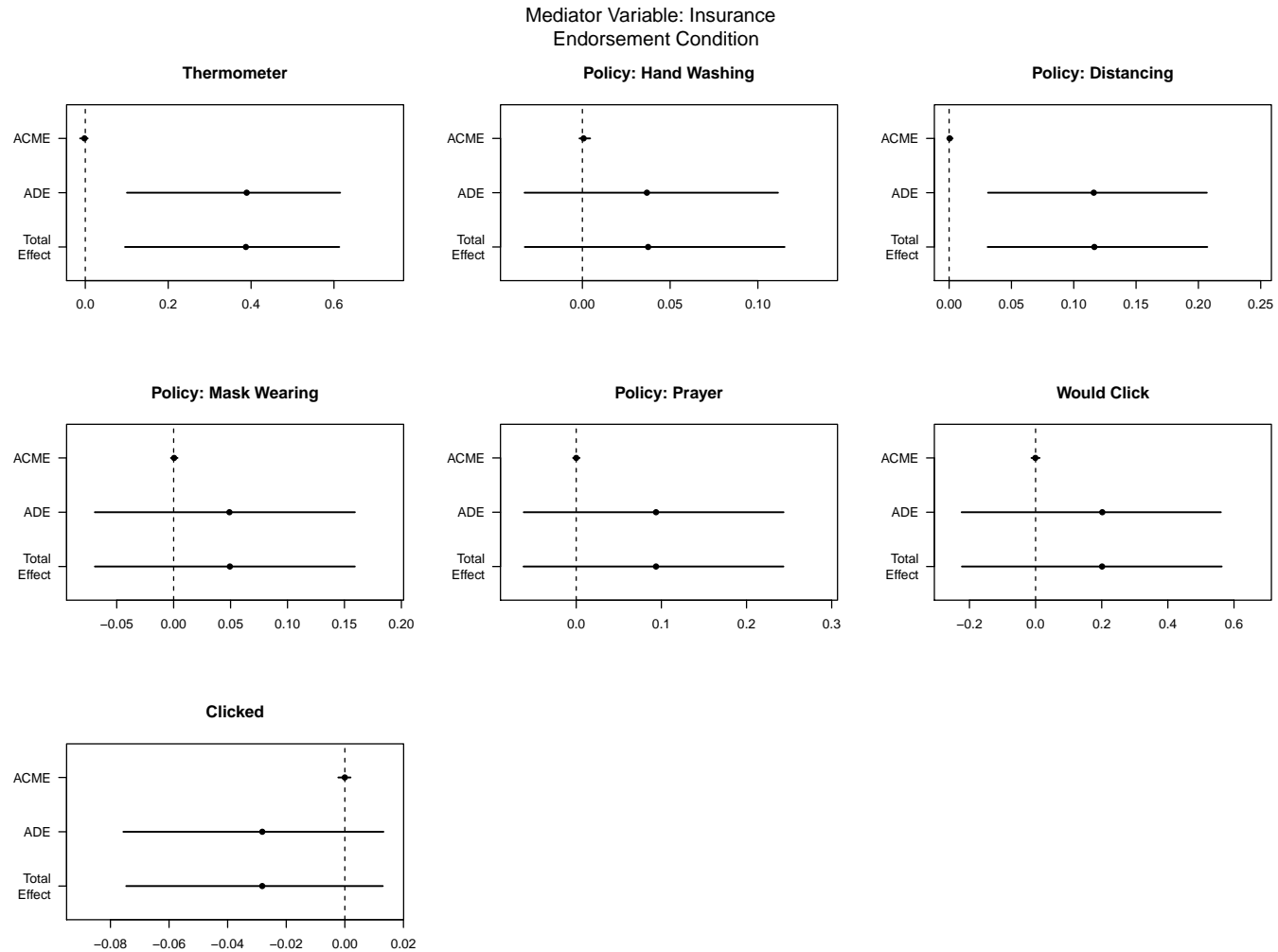


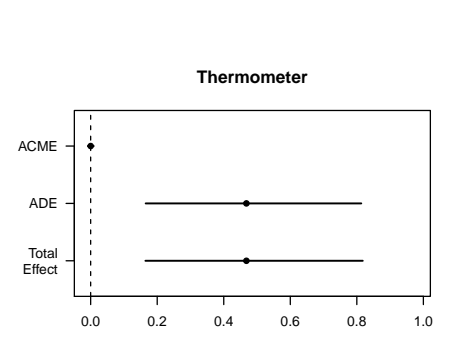

Mediator Variable: Insurance Norms Condition

Policy: Hand Washing
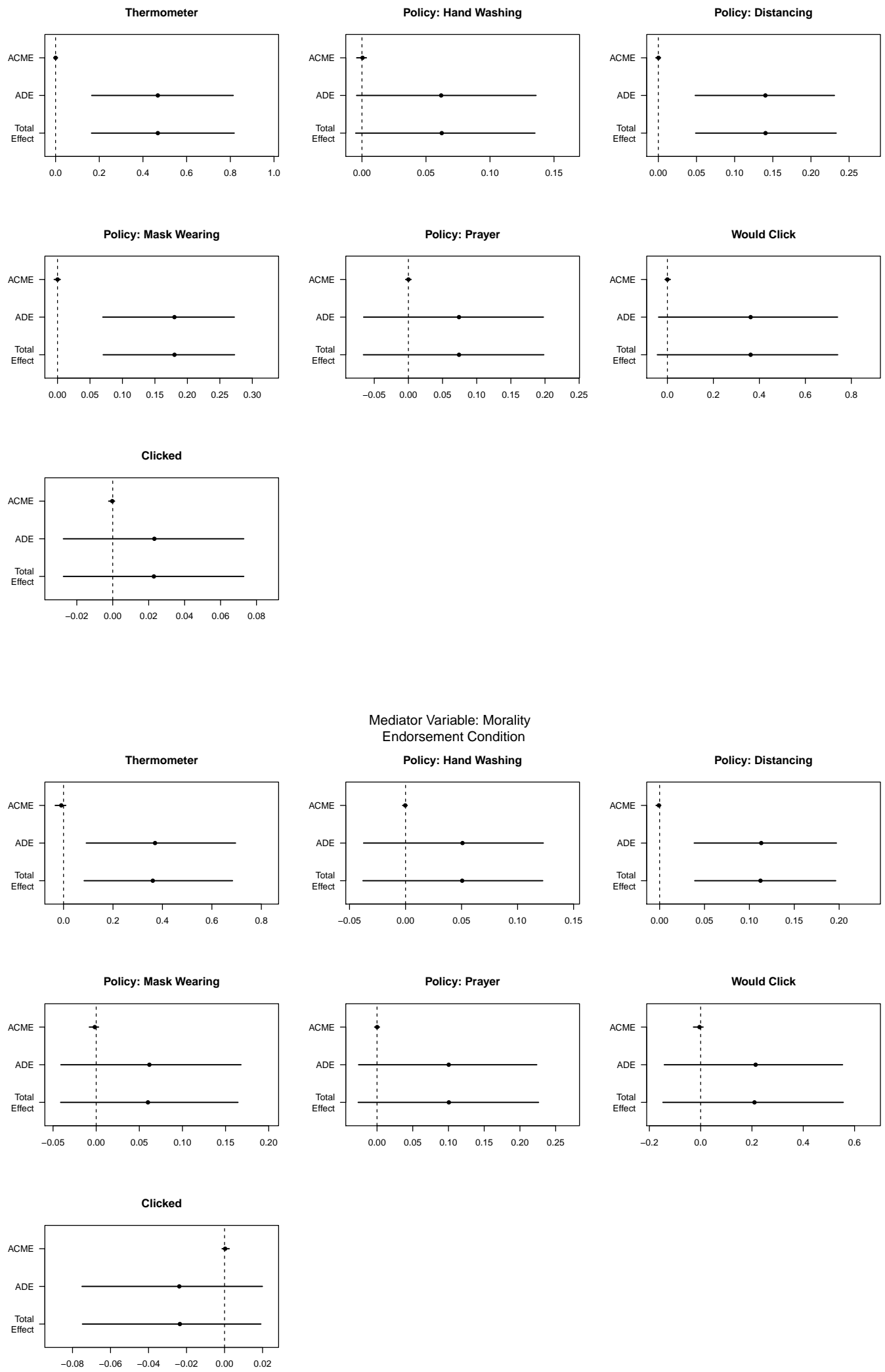

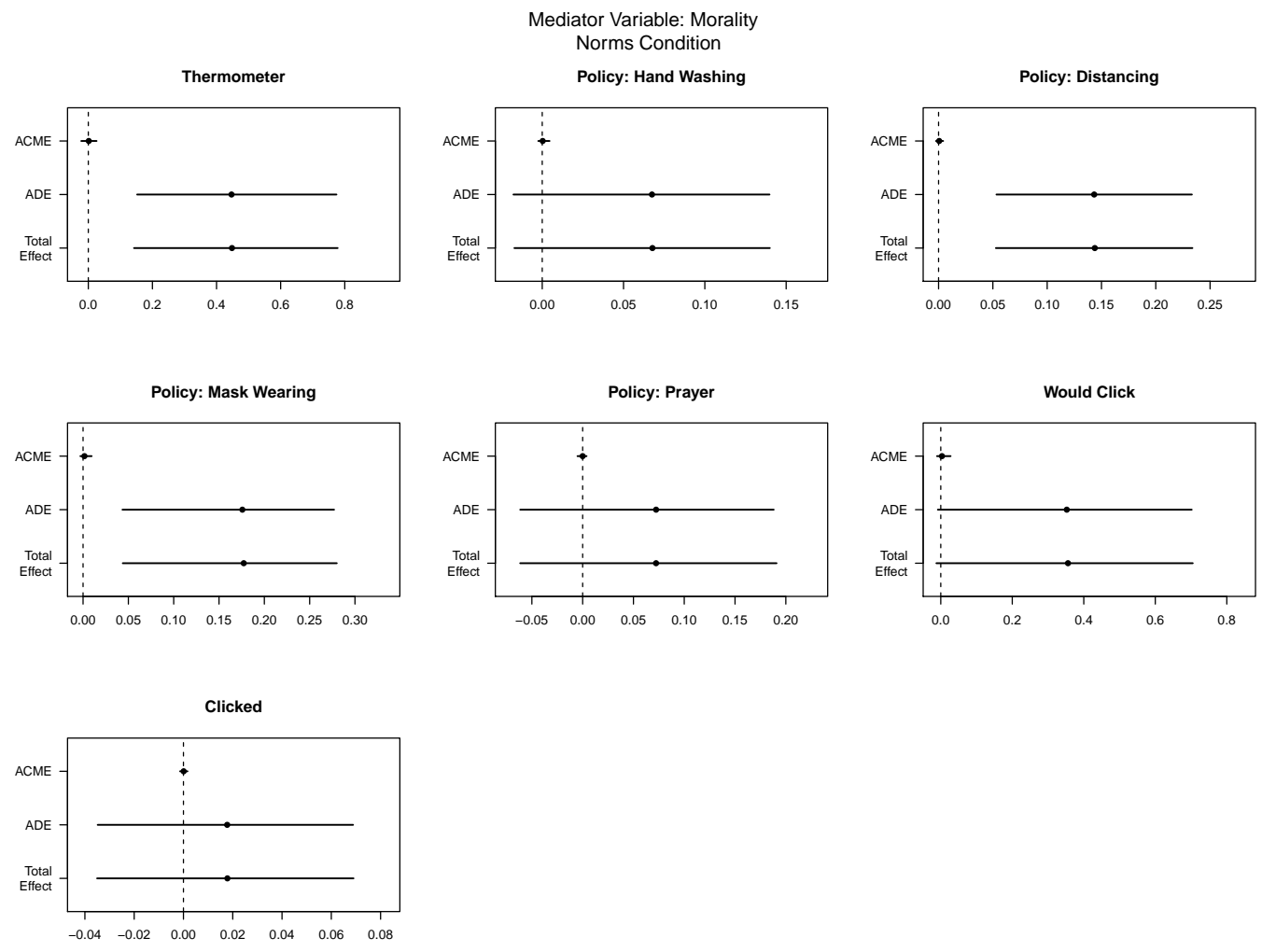

Mediator Variable: Social Capital Endorsement Condition
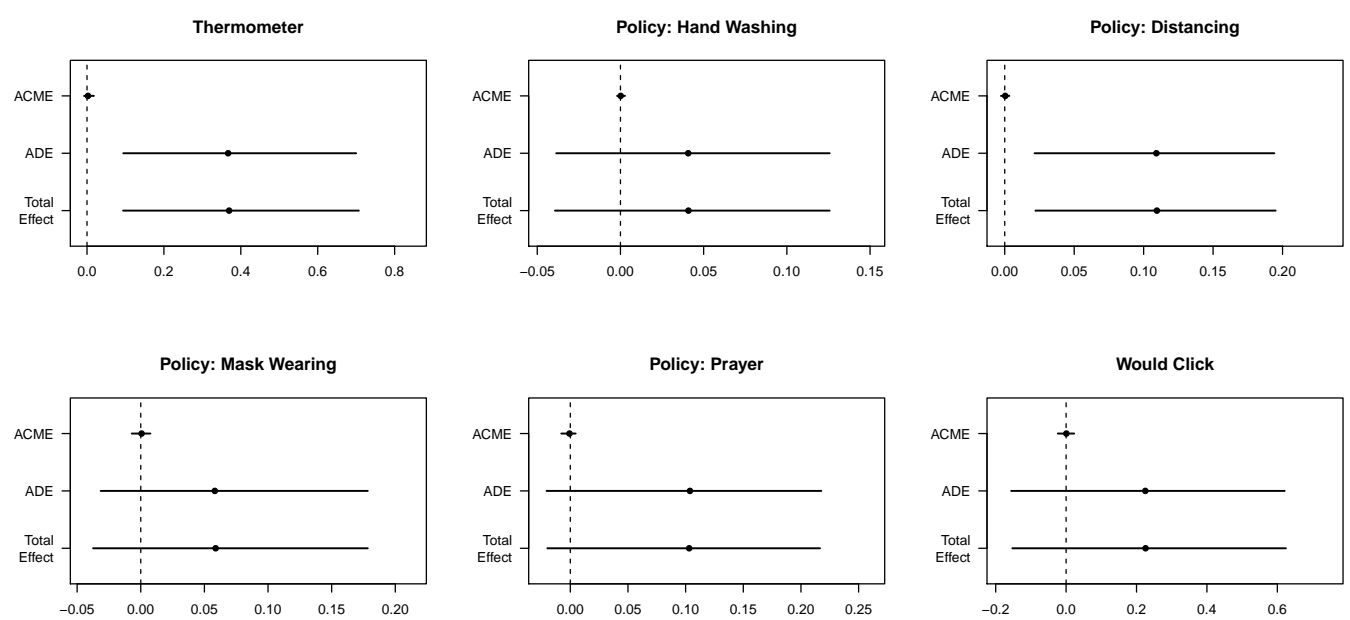

Clicked

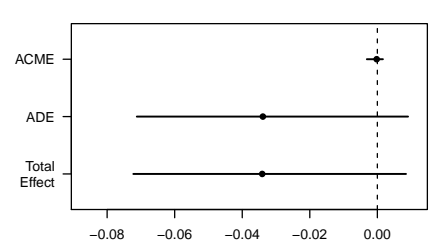




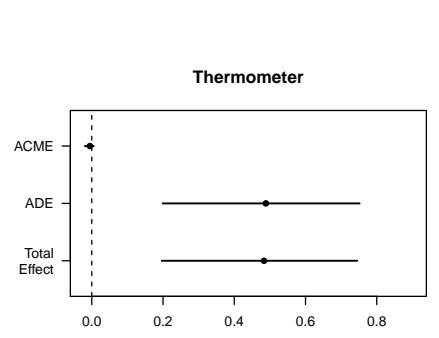

Mediator Variable: Social Capita

Norms Condition

Policy: Hand Washing
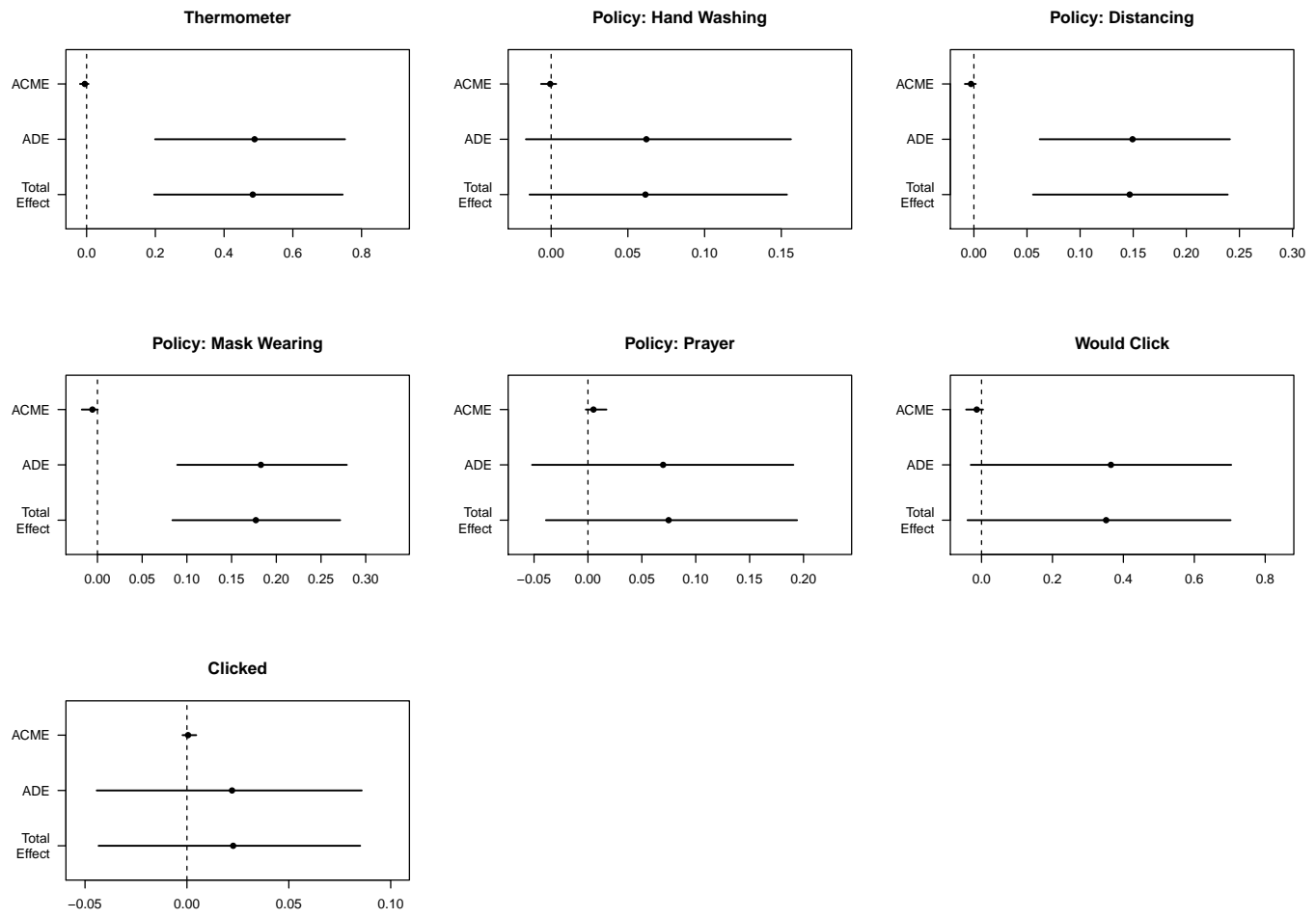

Mediator Variable: Heuristics Endorsement Condition
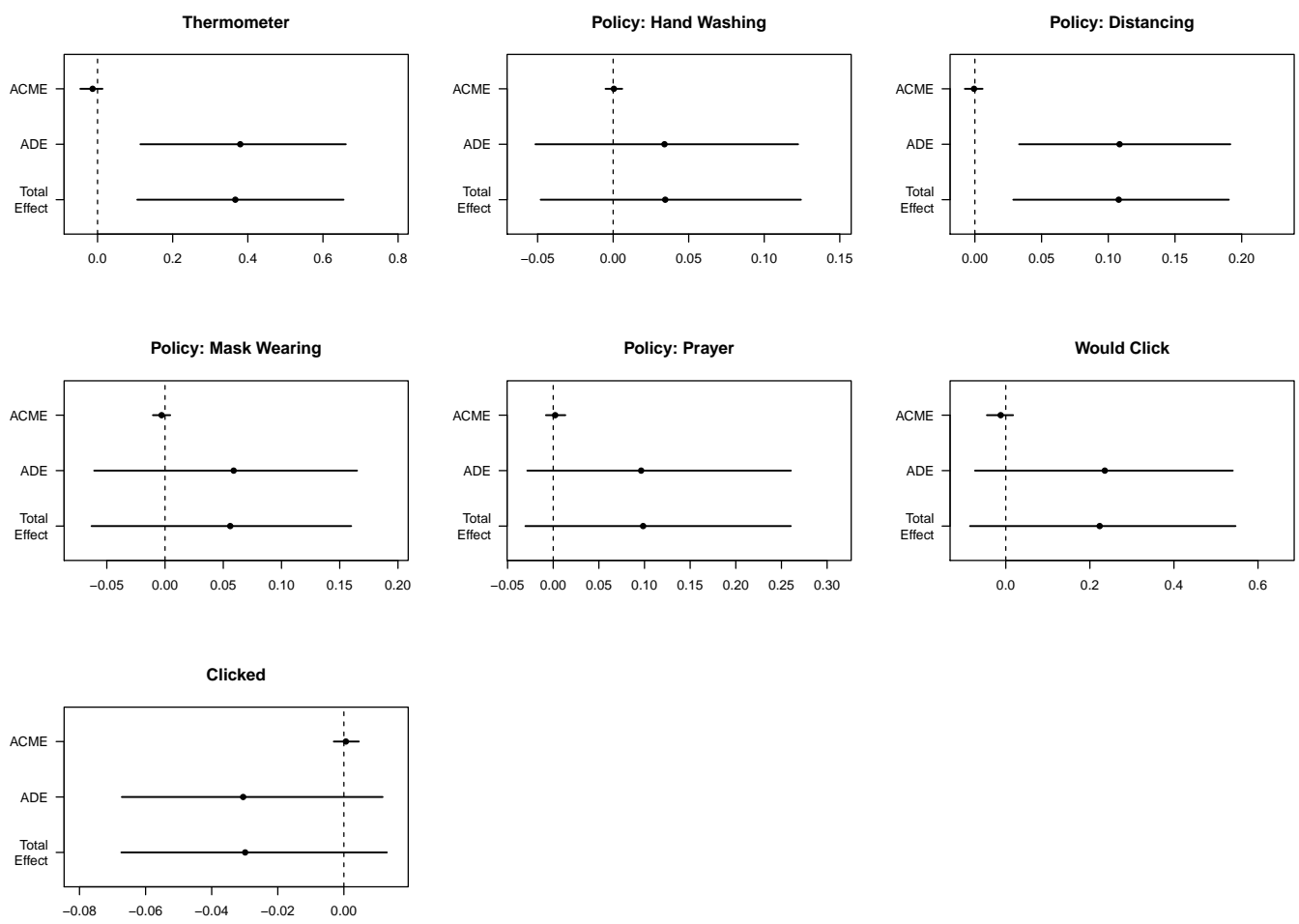


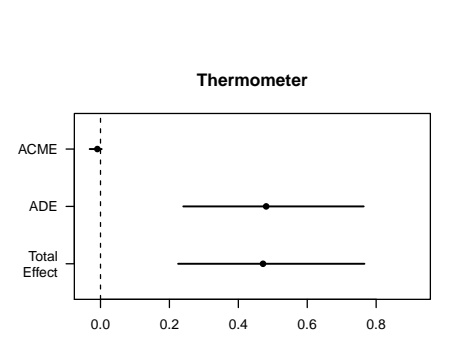

Mediator Variable: Heuristics Norms Condition

Policy: Hand Washing
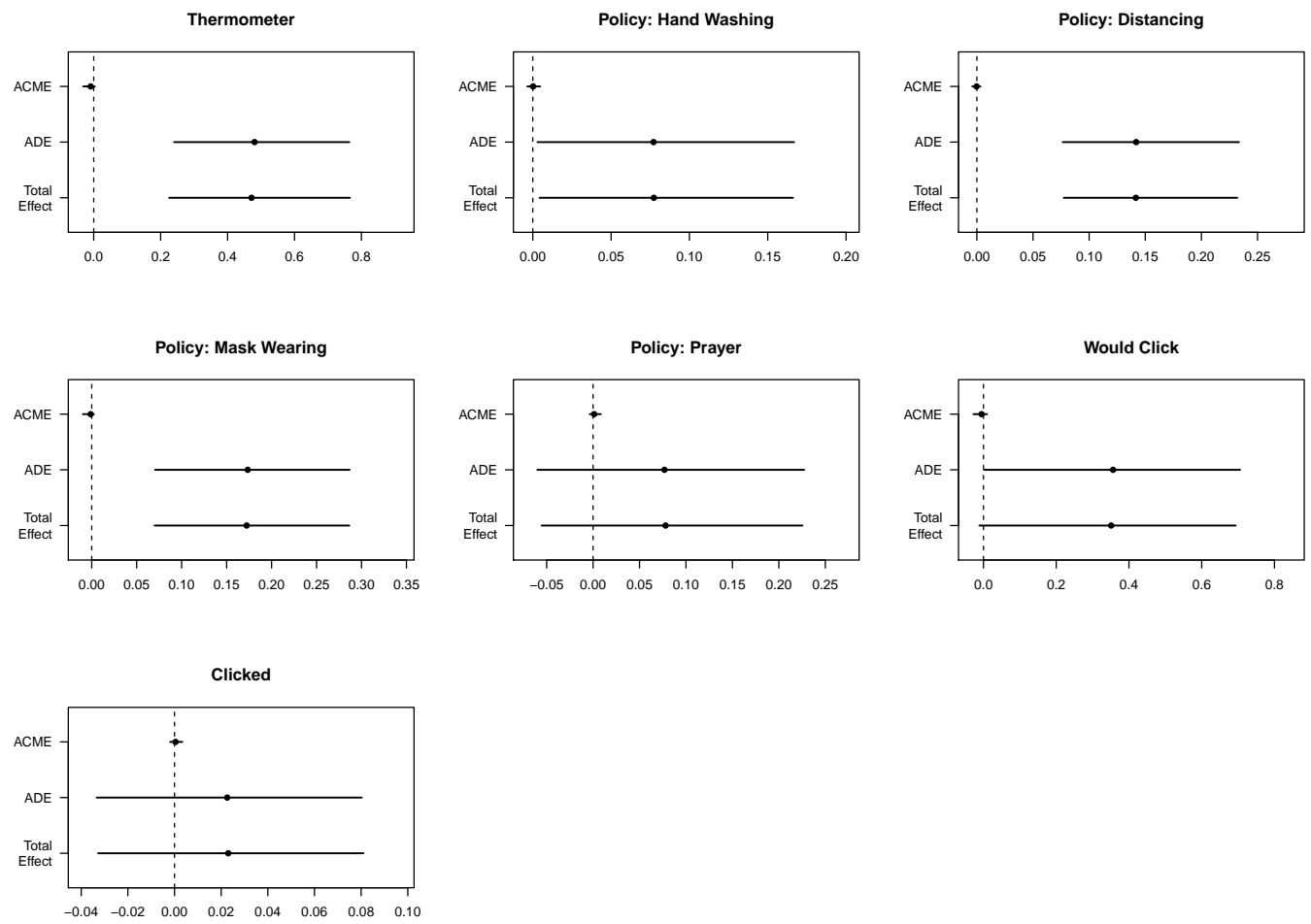

Mediator Variable: Tradition Endorsement Condition

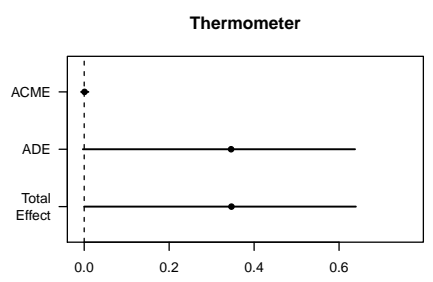

Policy: Hand Washing
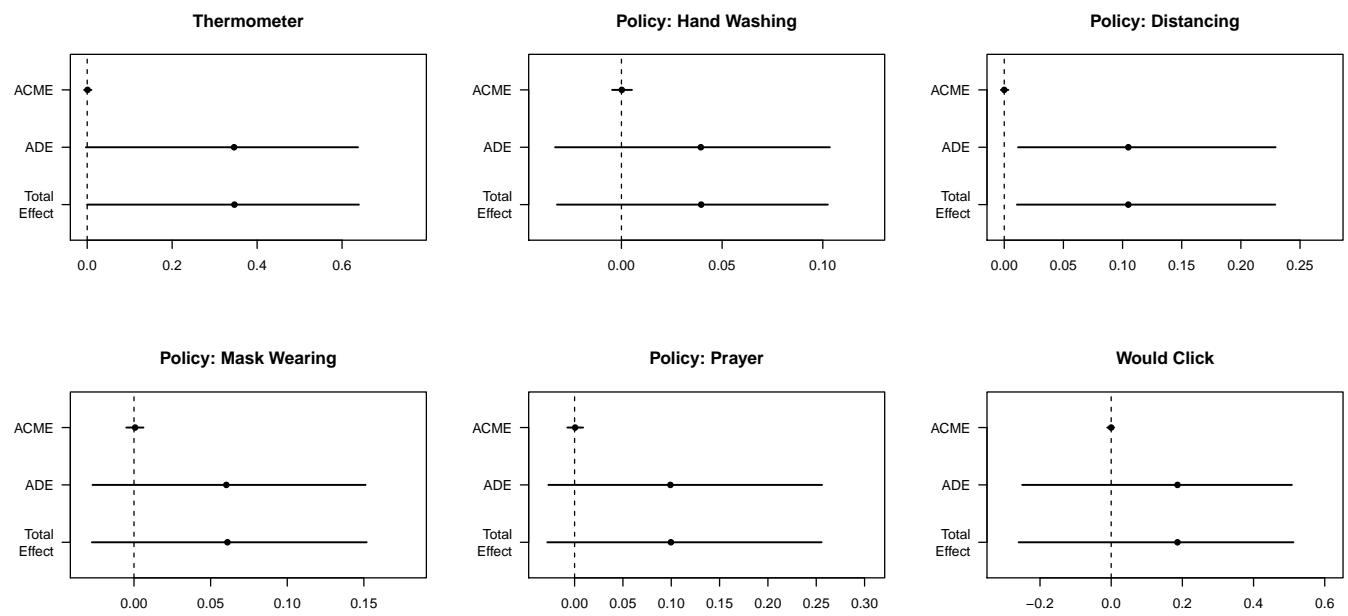

Clicked

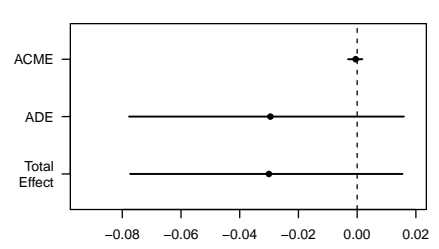


Mediator Variable: Tradition Norms Condition

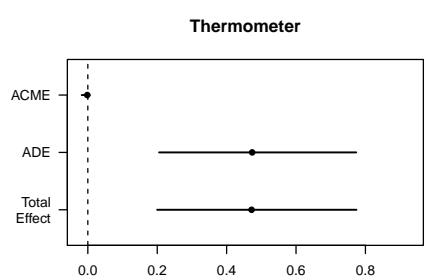

Policy: Hand Washing
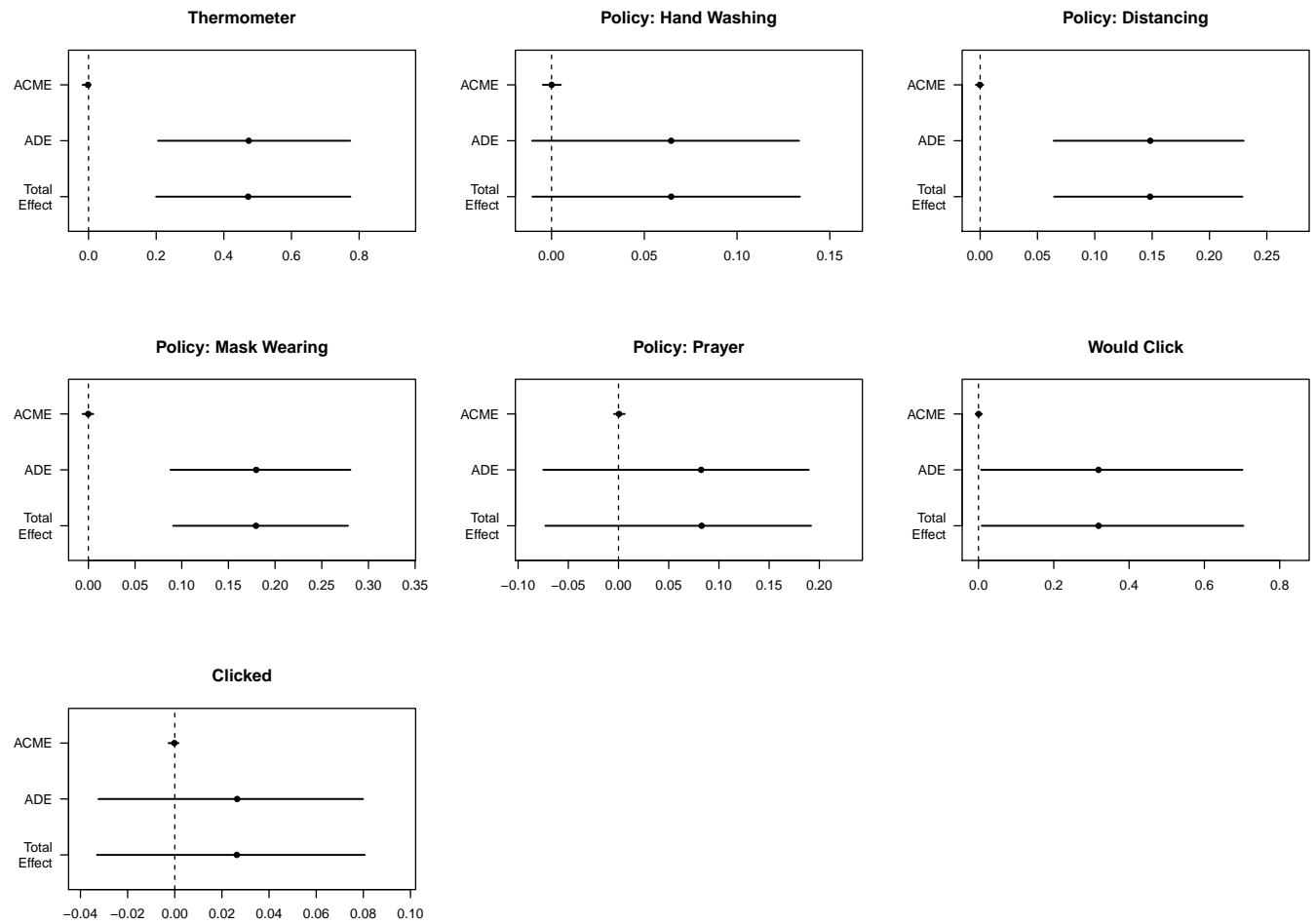

Mediator Variable: Social Identity Endorsement Condition

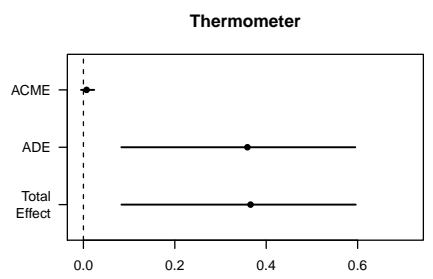

Policy: Hand Washing

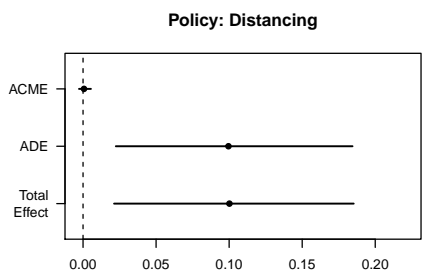

Policy: Mask Wearing
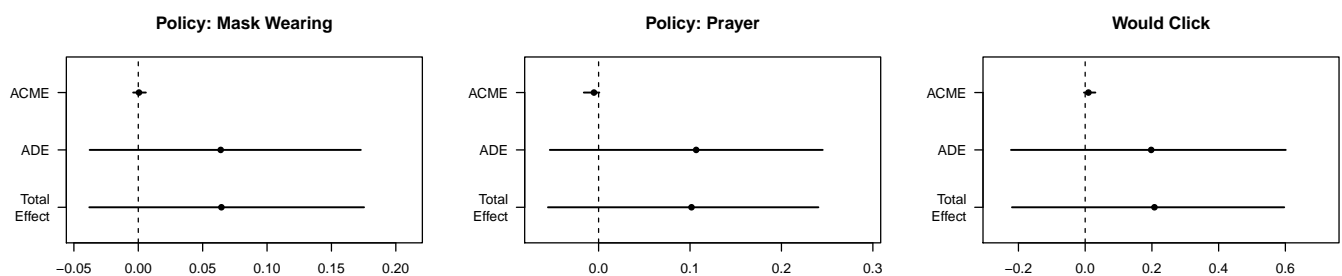

Clicked
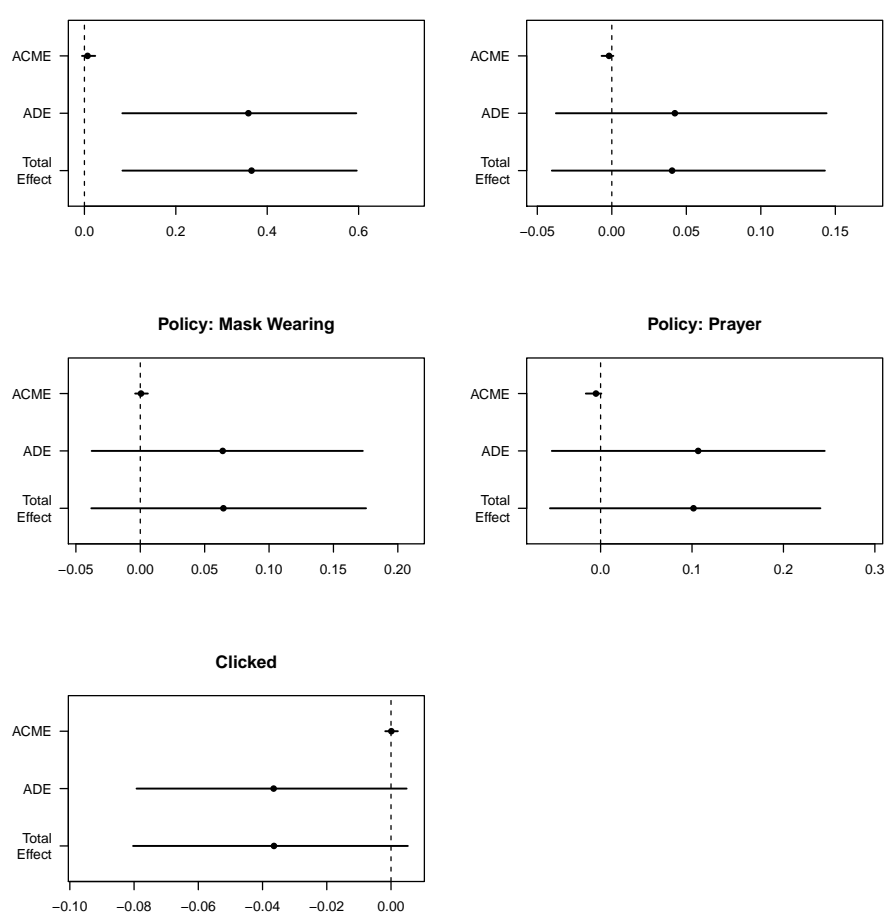
Mediator Variable: Social Identity Norms Condition

Thermometer

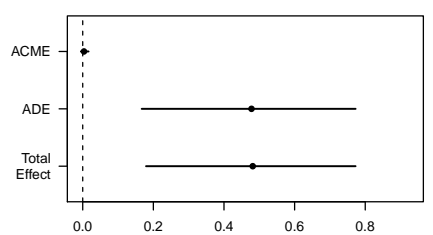

Policy: Mask Wearing

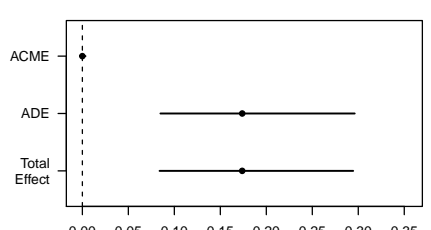

$\begin{array}{llllllll}0.00 & 0.05 & 0.10 & 0.15 & 0.20 & 0.25 & 0.30 & 0.35\end{array}$

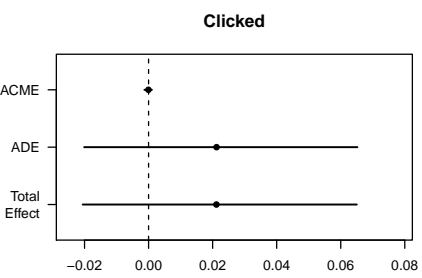

Policy: Hand Washing

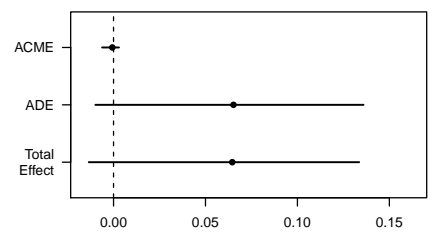

Policy: Prayer

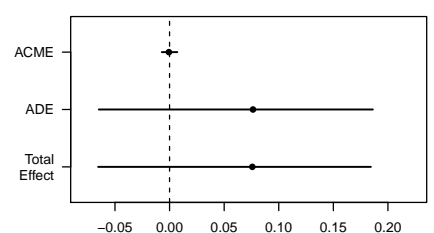

Policy: Distancing

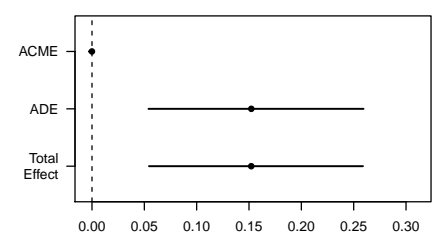

Would Click

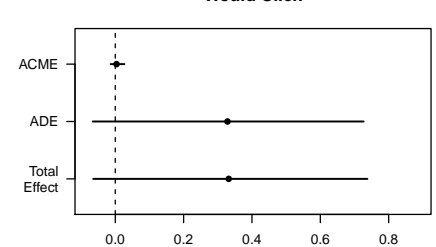


F.3 Extrinsic / Intrinsic Motivations

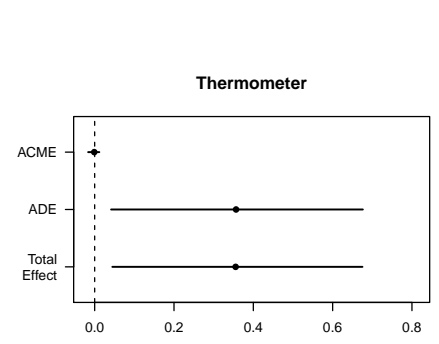

Mediator Variable: Religion Offers Comfort Endorsement Condition
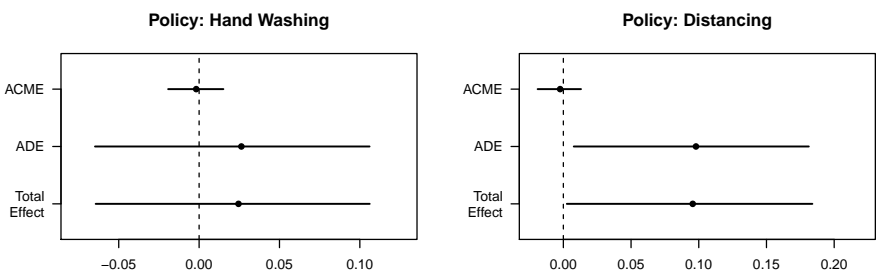

Policy: Mask Wearing

Policy: Prayer
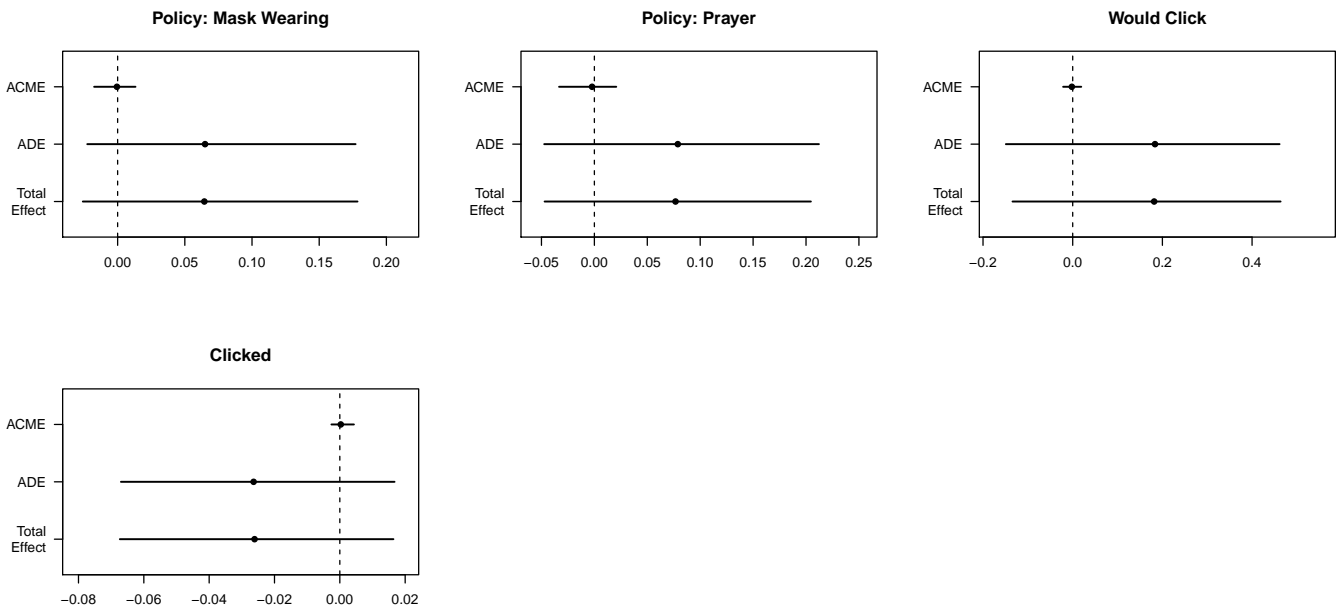
Thermometer

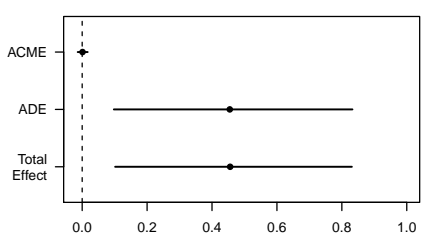

Policy: Mask Wearing

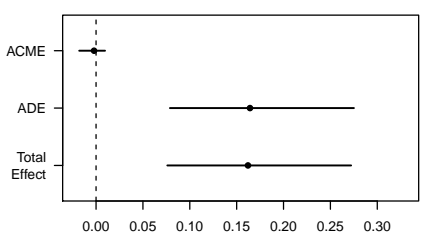

Clicked

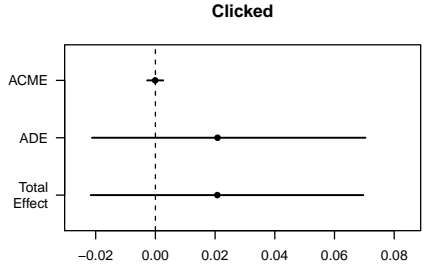

Thermometer

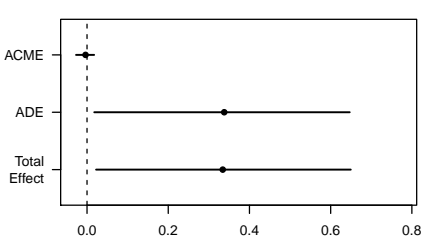

Policy: Mask Wearing

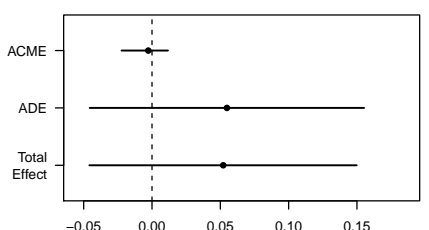

Clicked

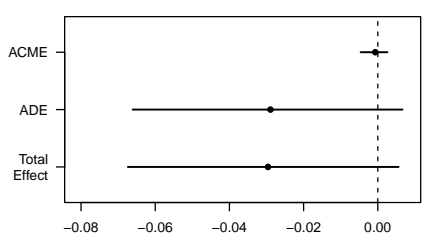

Mediator Variable: Religion Offers Comfort Norms Condition

Policy: Hand Washing
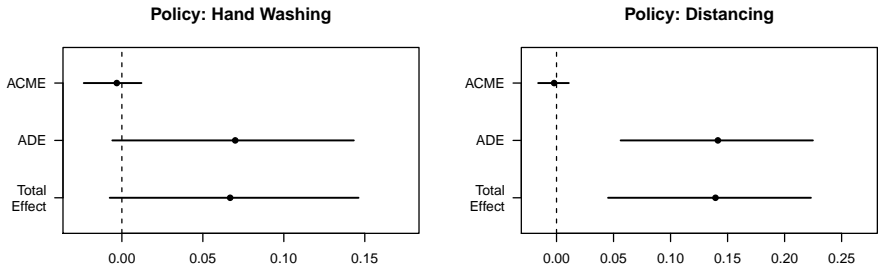

Policy: Prayer
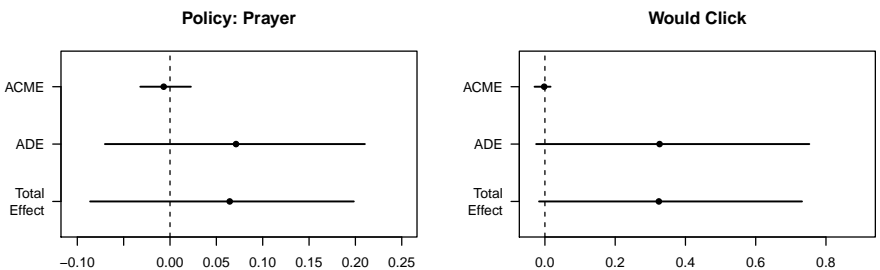

Mediator Variable: Religious Approach to Life Endorsement Condition

Policy: Hand Washing

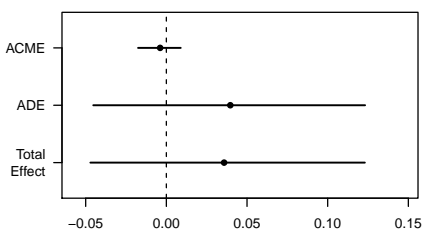

Policy: Prayer
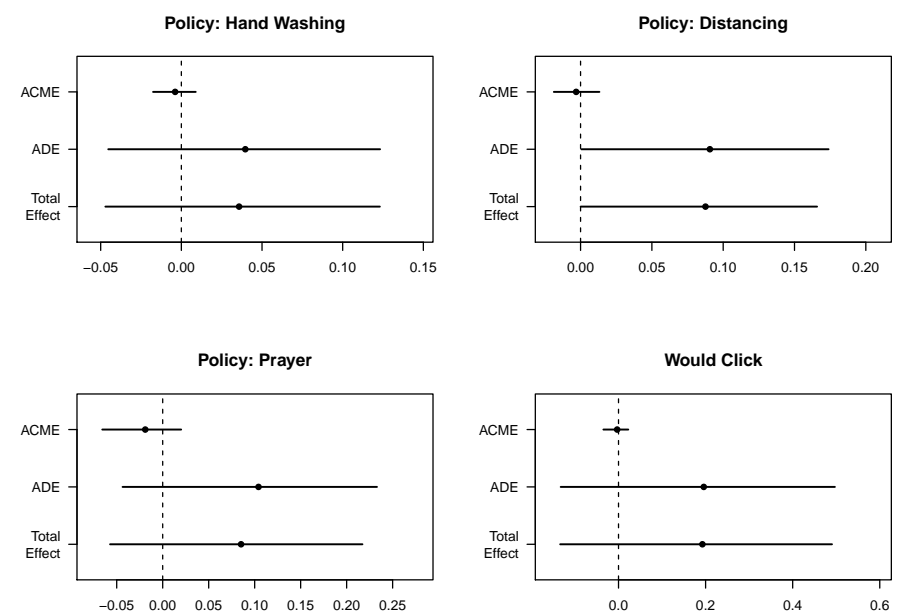


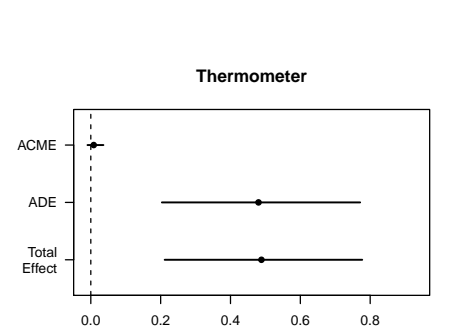

Mediator Variable: Religious Approach to Life Norms Condition
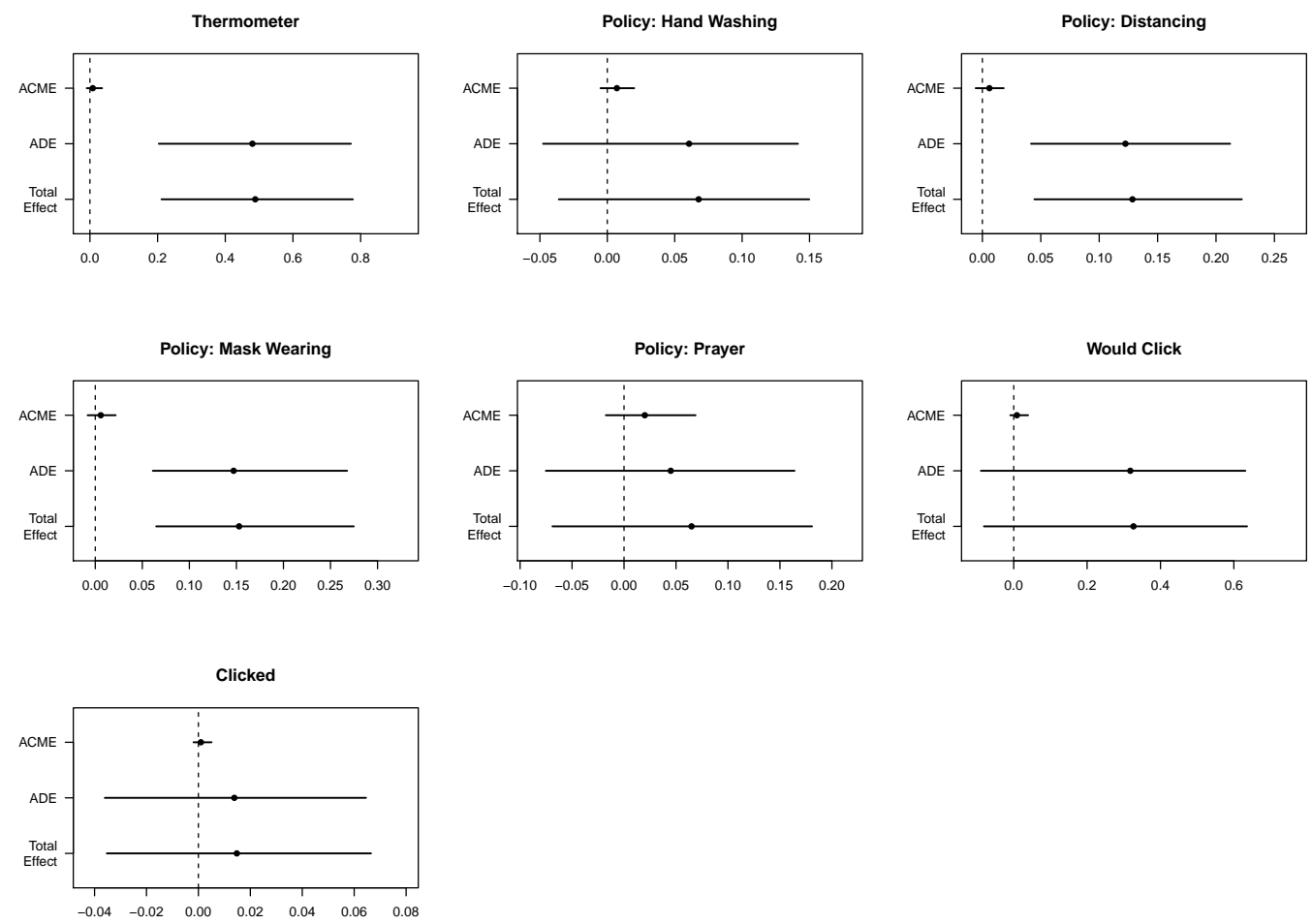

Mediator Variable: Enjoys Social Aspects of Church

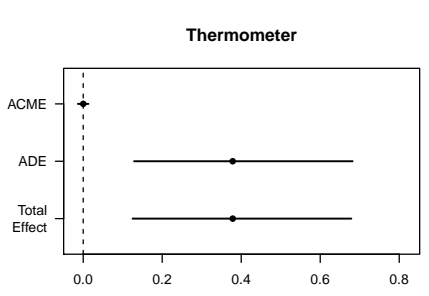
Endorsement Condition
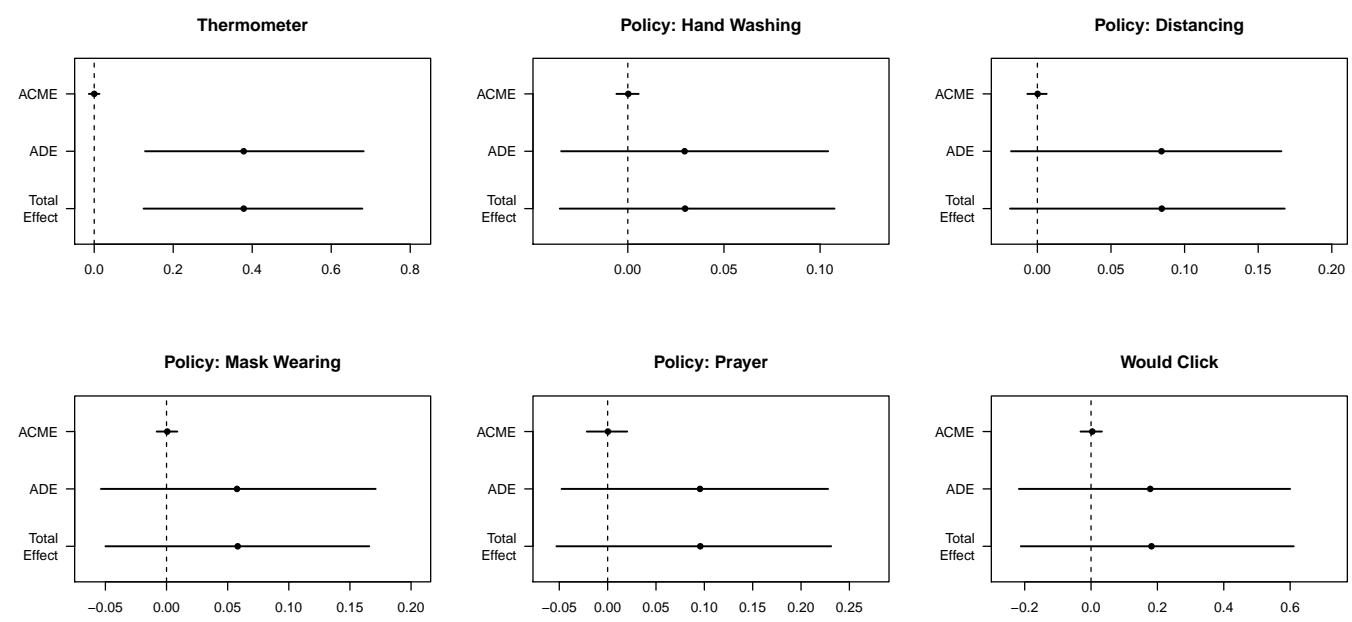

Clicked

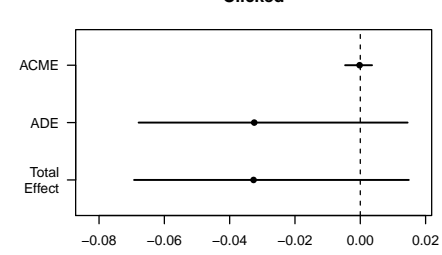




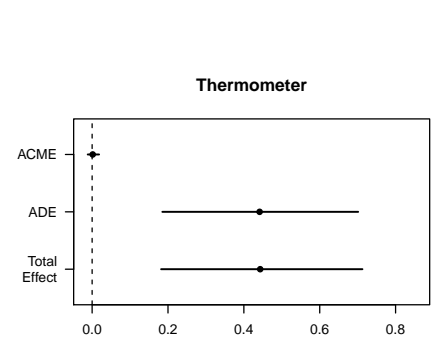

Mediator Variable: Enjoys Social Aspects of Church Norms Condition

Policy: Hand Washing

Policy: Distancing
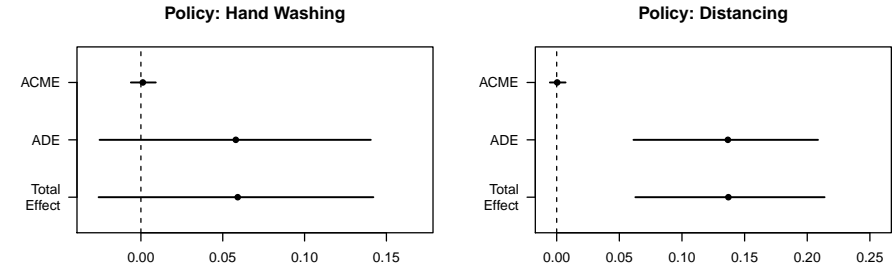

Policy: Mask Wearing

Policy: Prayer
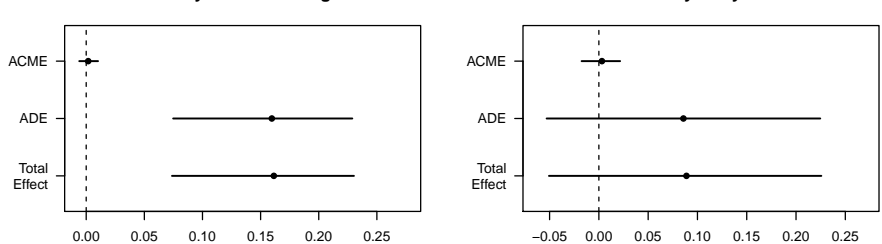

Would Click
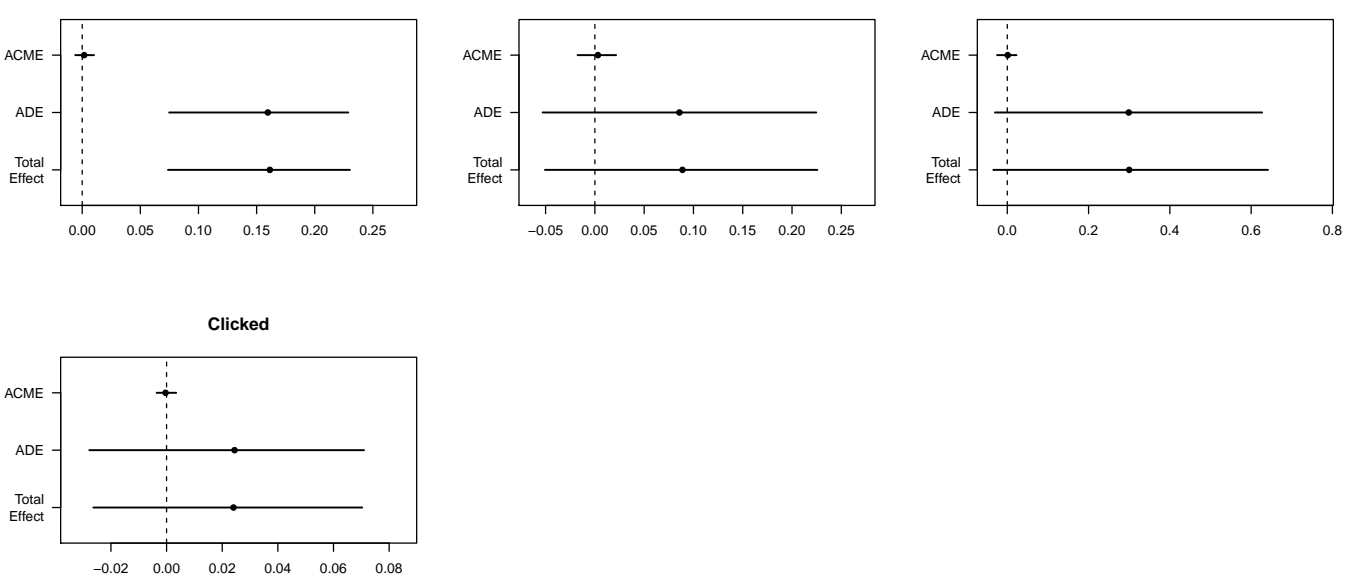


\section{F.4 Religiosity Index}

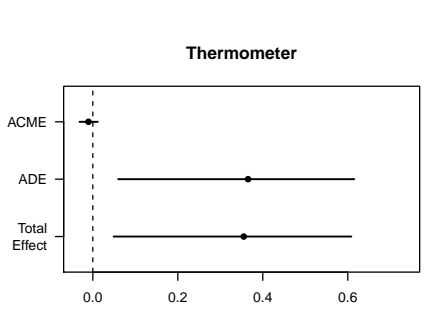

Mediator Variable: Regularly Attends Church Endorsement Condition

Policy: Hand Washing

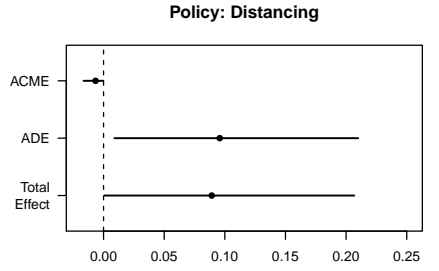

Policy: Mask Wearing

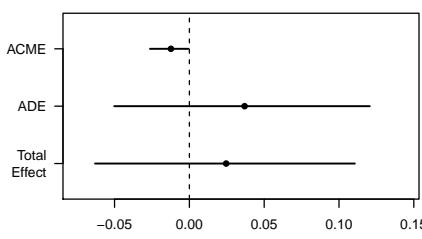

Policy: Prayer
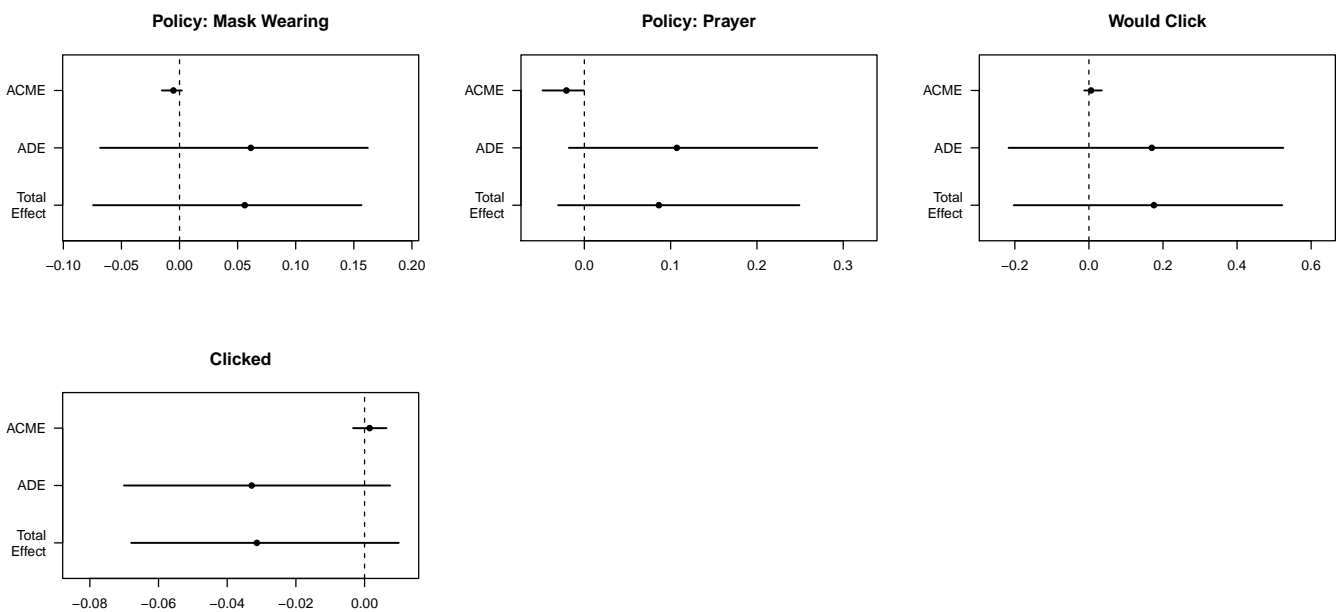


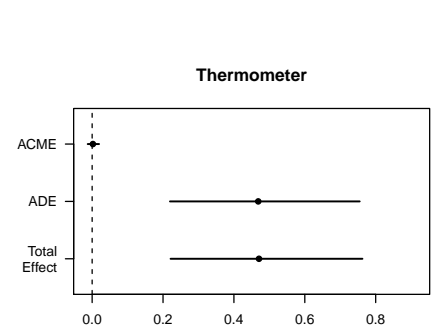

Mediator Variable: Regularly Attends Church Norms Condition

Policy: Hand Washing

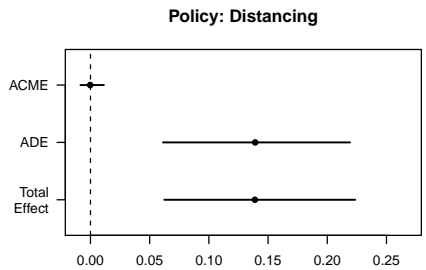

Policy: Mask Wearing
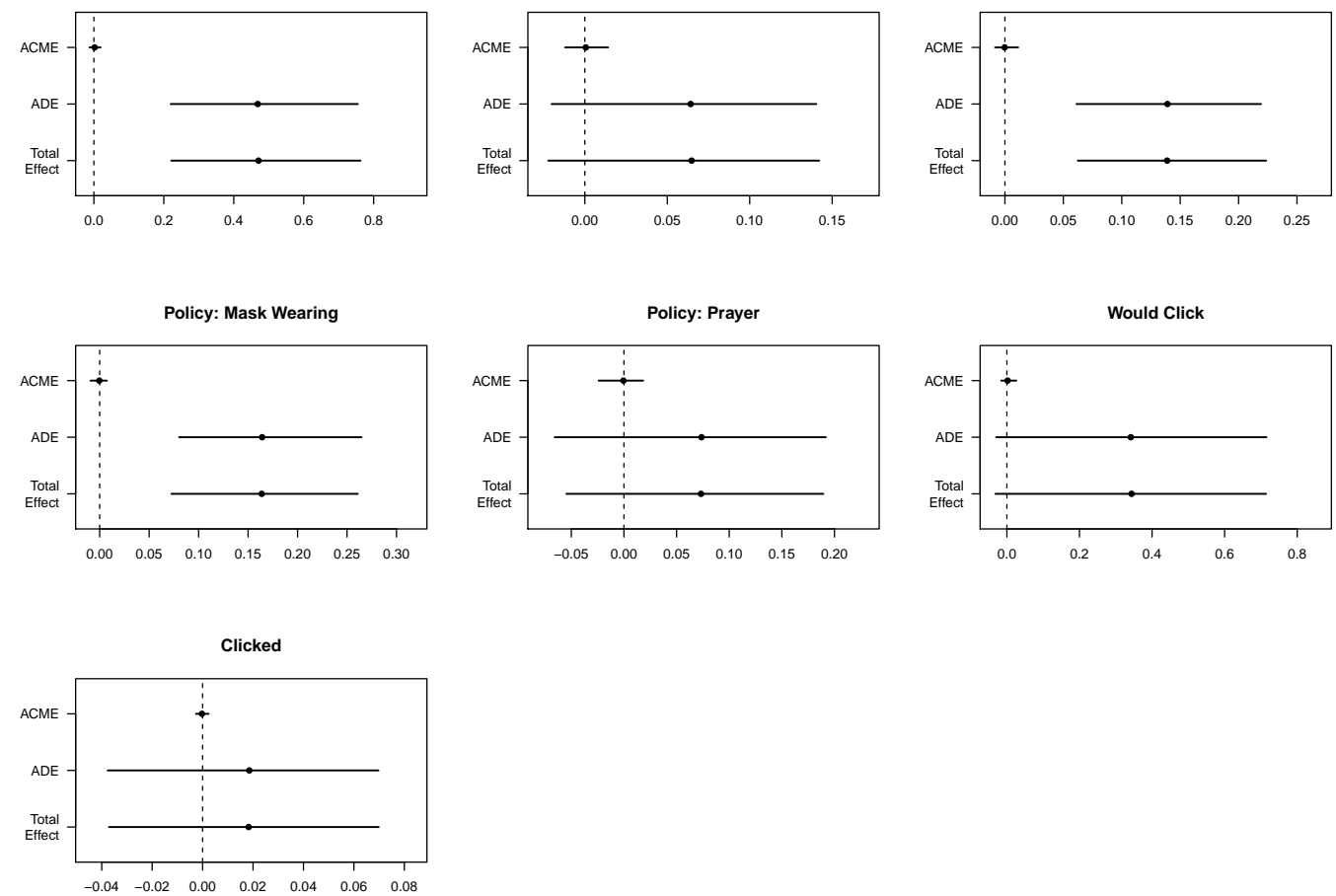

Mediator Variable: Spiritual Values Endorsement Condition
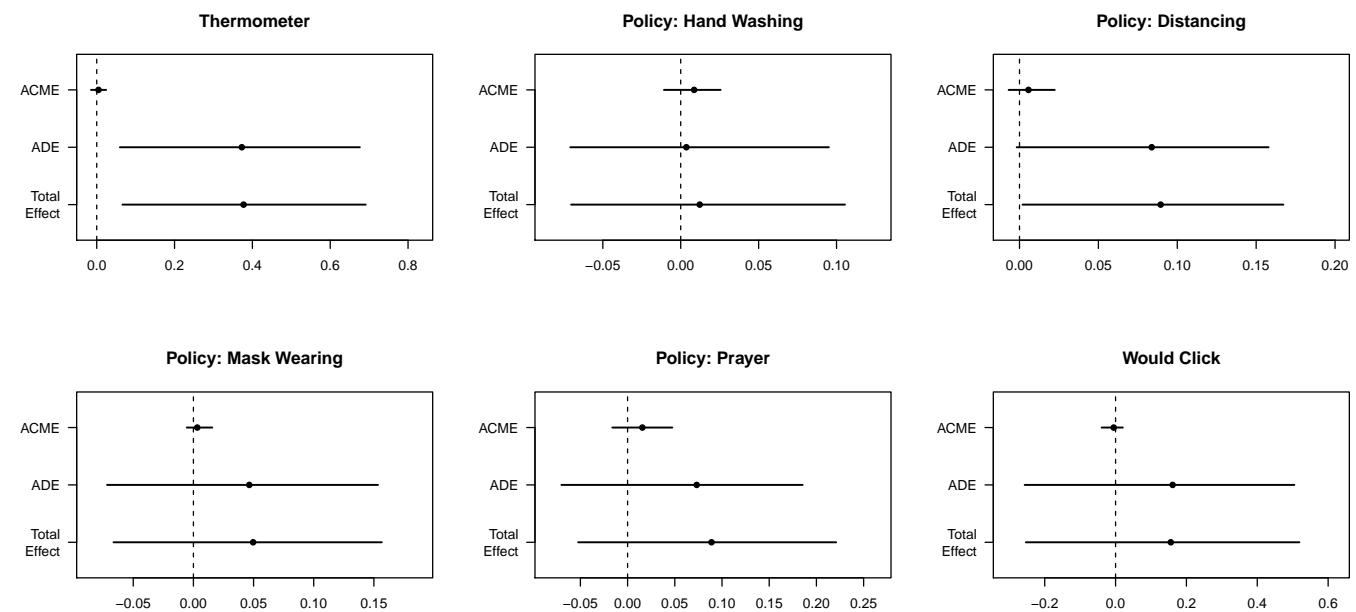

Clicked

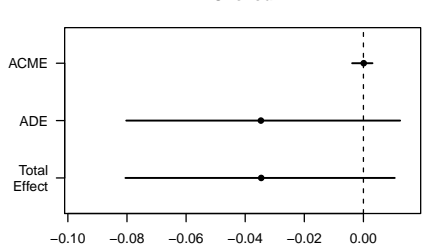




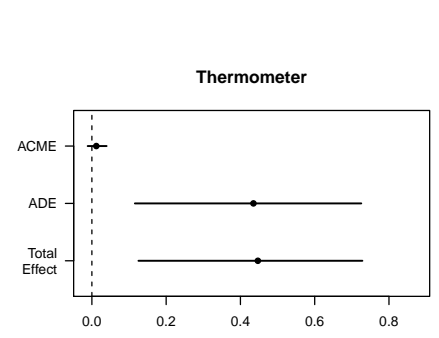

Mediator Variable: Spiritual Values Norms Condition

Policy: Hand Washing

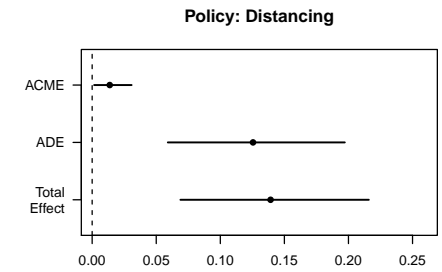

Policy: Mask Wearing
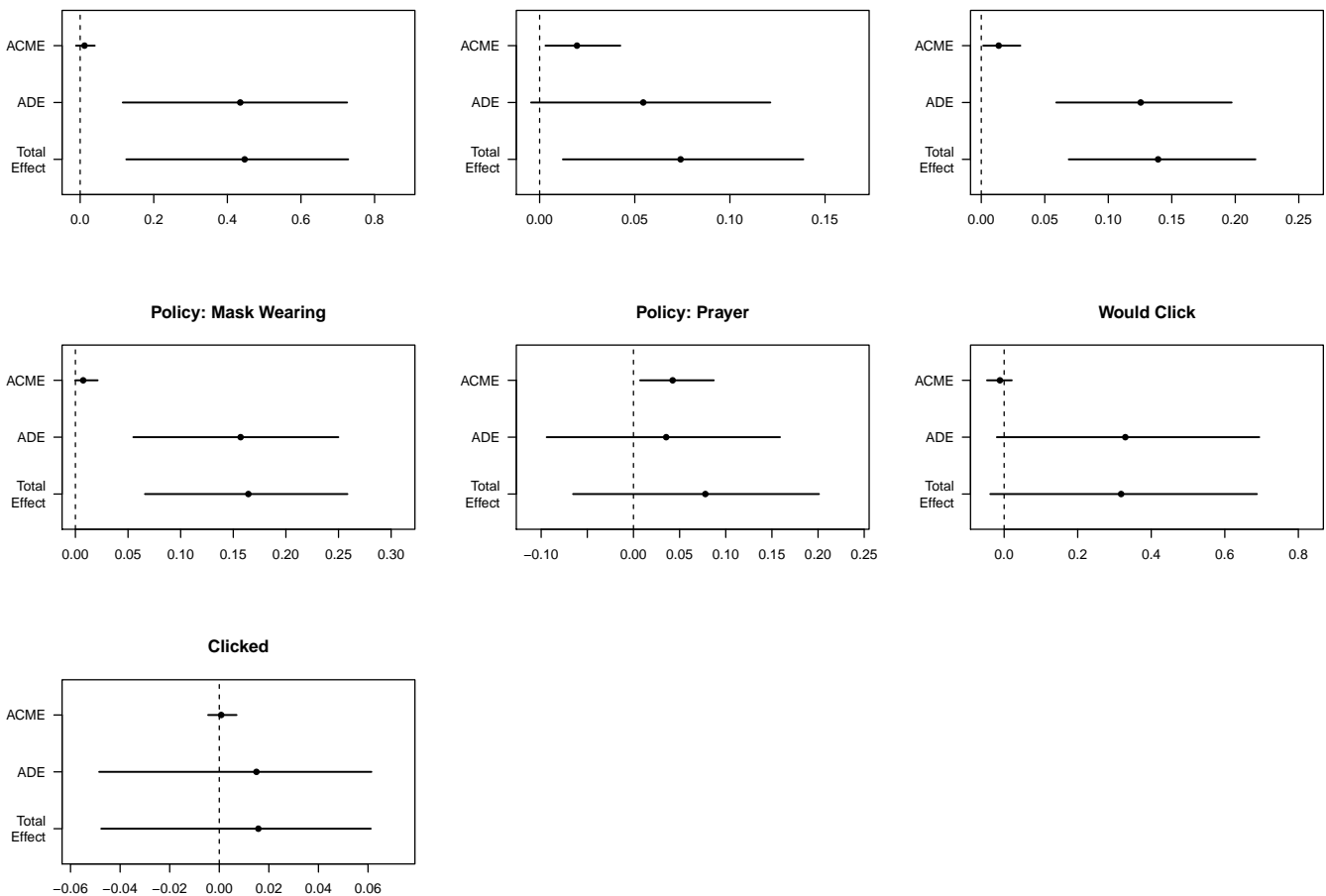

Mediator Variable: Enjoys Social Aspects of Church Endorsement Condition

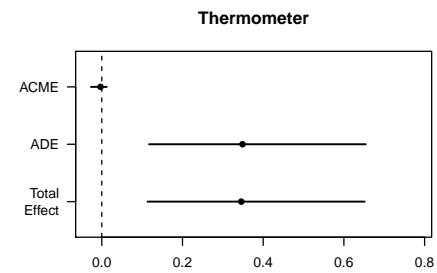

Policy: Hand Washing

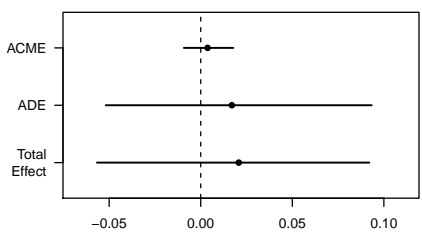

Policy: Prayer

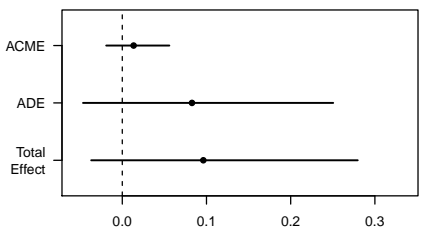

Policy: Distancing
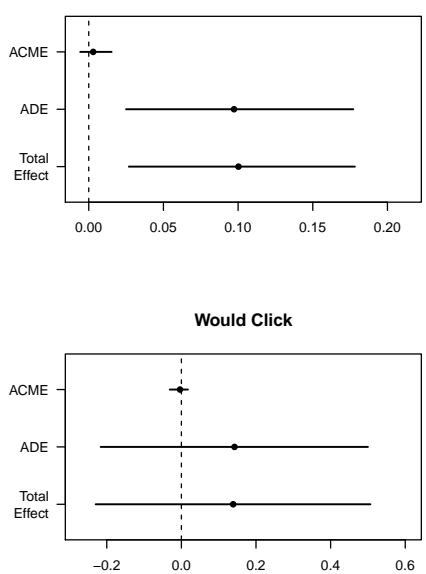

Clicked

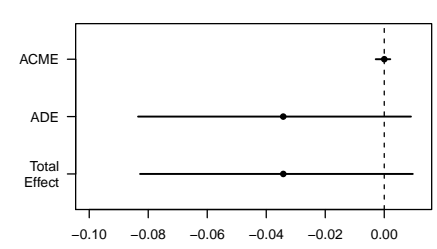




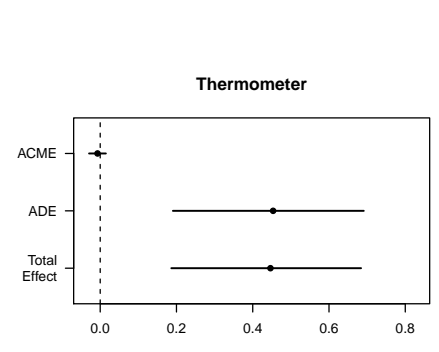

Mediator Variable: Enjoys Social Aspects of Church Norms Condition

Policy: Hand Washing
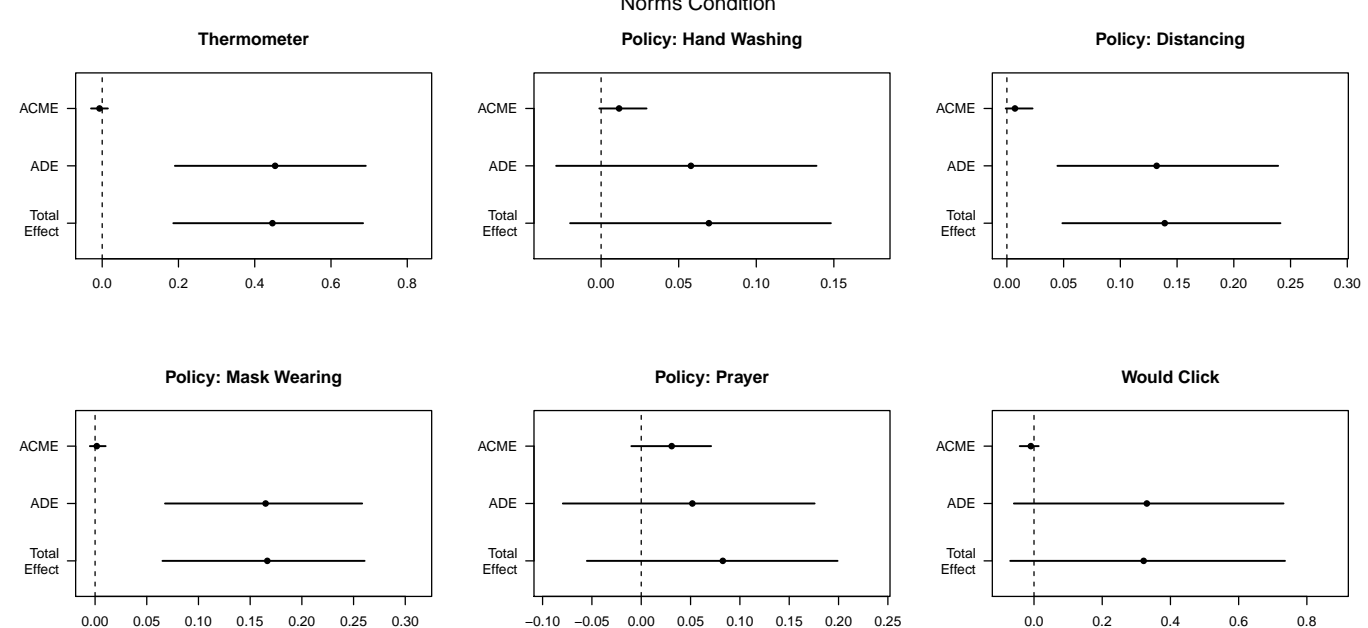

Would Click
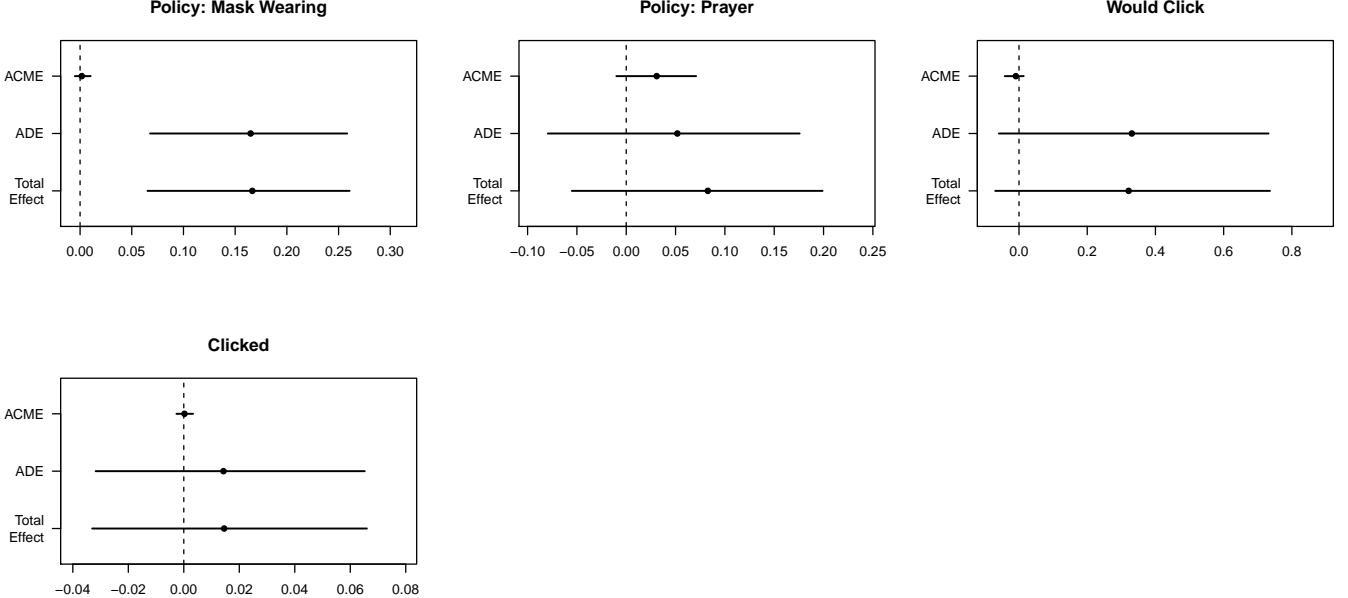


\section{G Interaction Models}

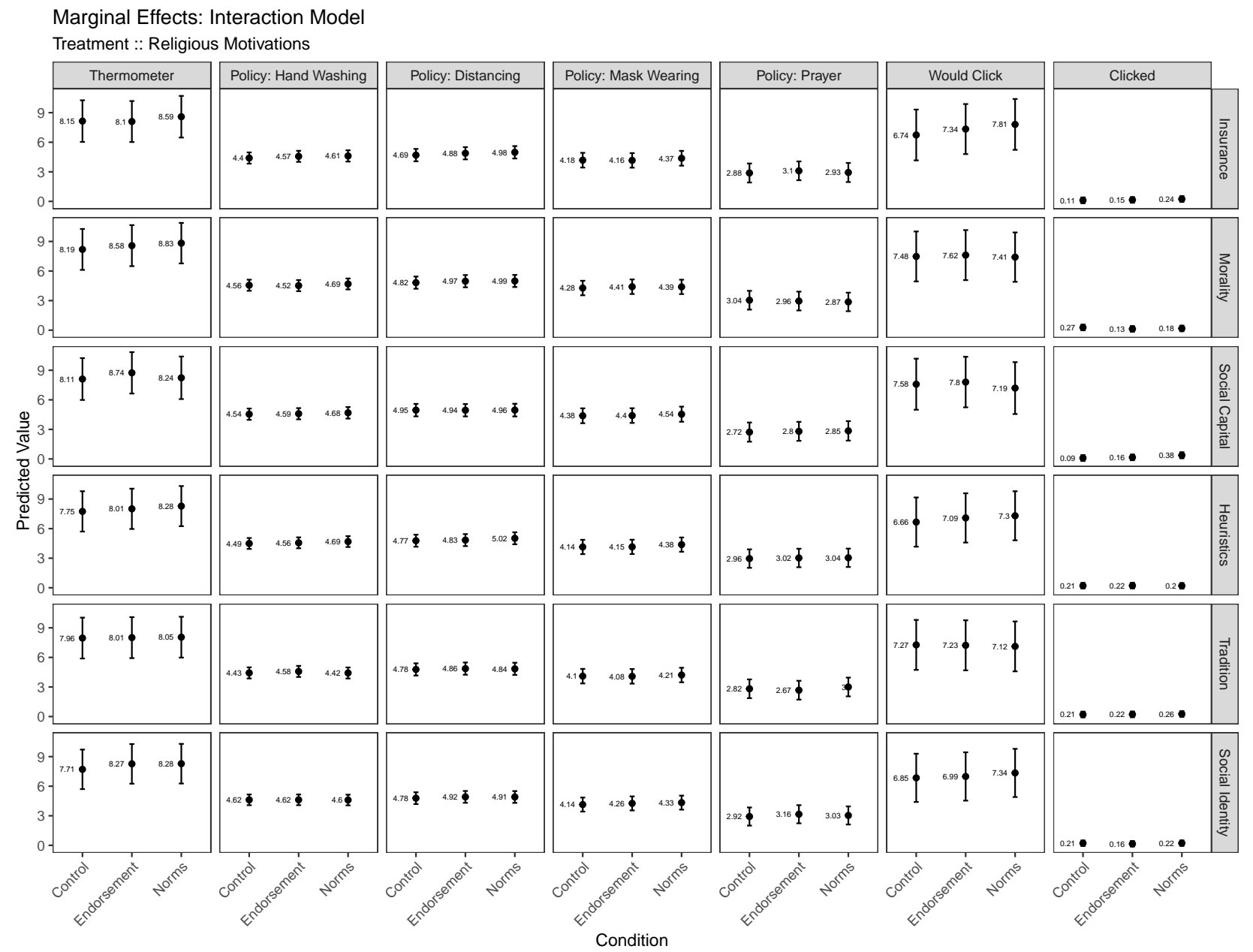

Figure G5: Predicted Values by Interaction (Treatment $\times$ Religious Motivations) 


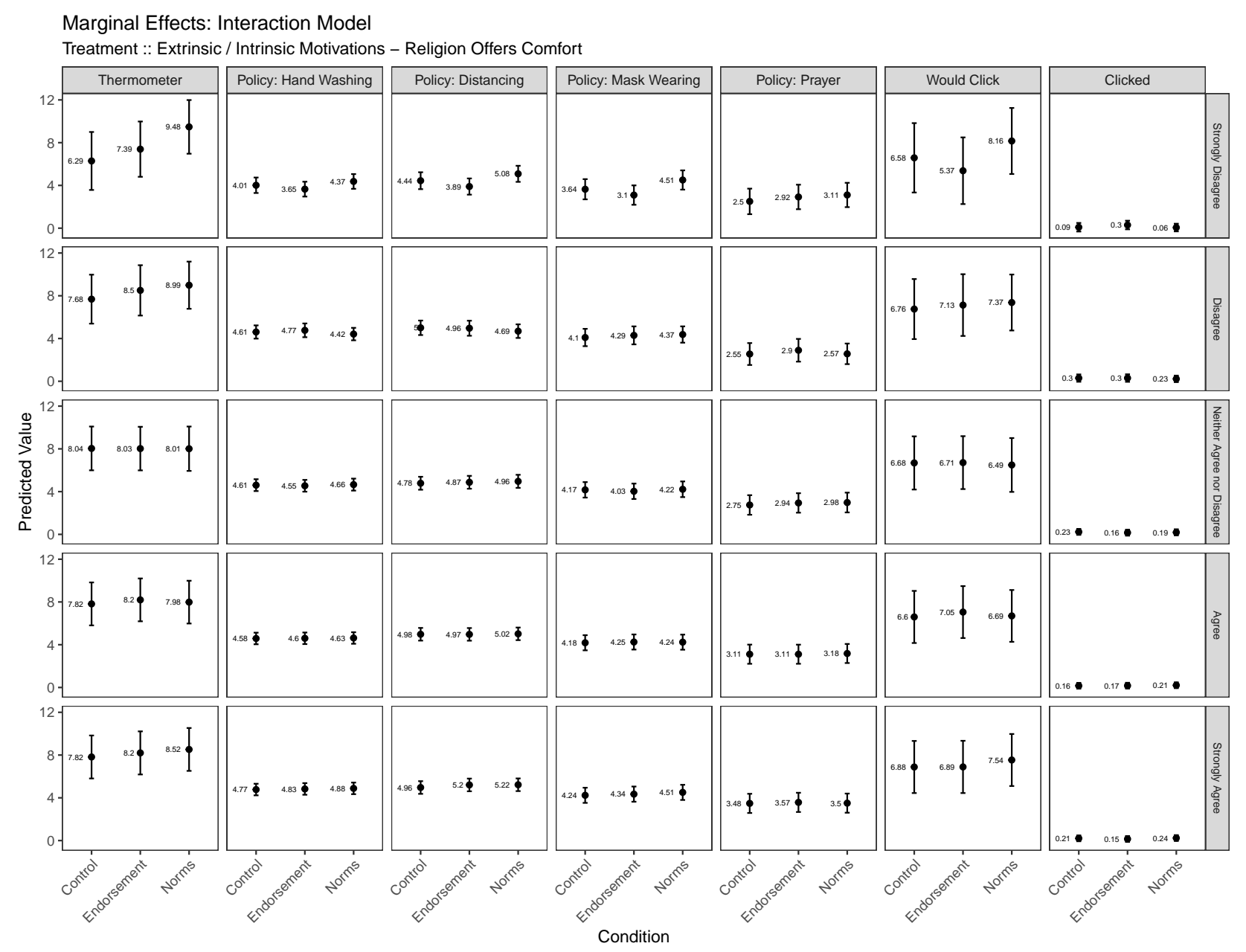

Figure G6: Predicted Values by Interaction (Treatment $\times$ Extrinsic / Intrinsic Motivations) 


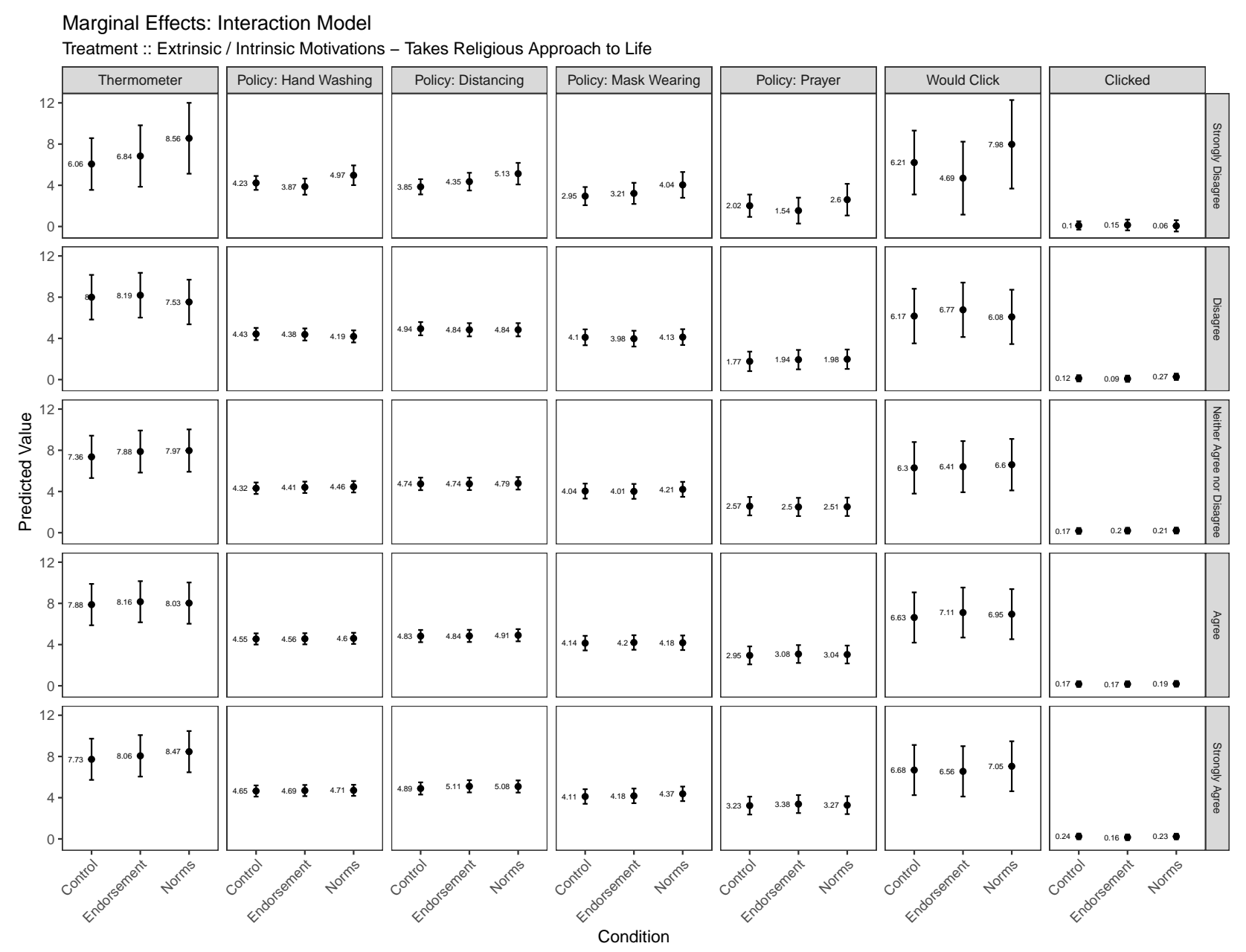

Figure G7: Predicted Values by Interaction (Treatment $\times$ Extrinsic / Intrinsic Motivations) 
Marginal Effects: Interaction Model

Treatment :: Extrinsic / Intrinsic Motivations - Enjoys Social Aspects of Church

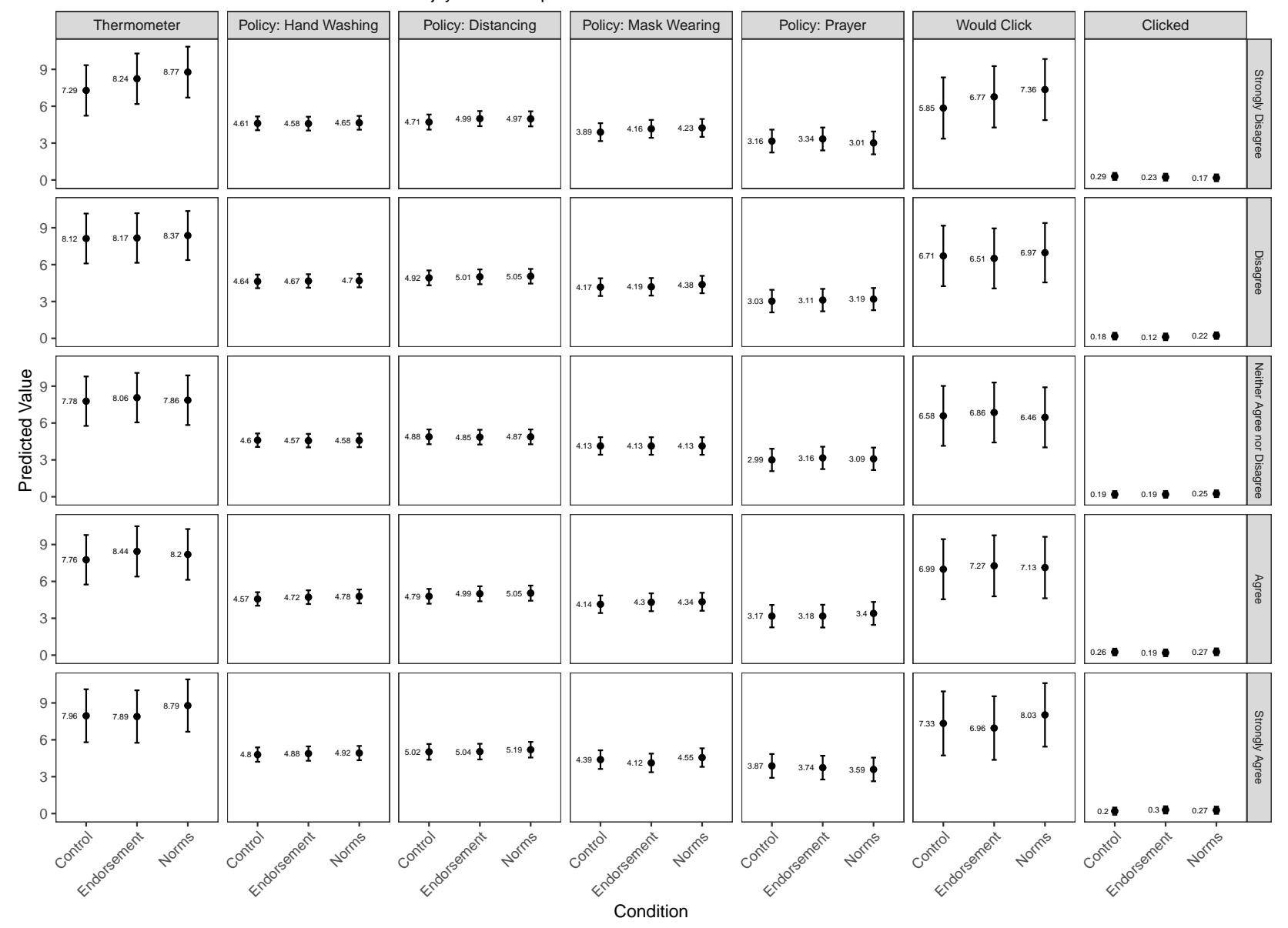

Figure G8: Predicted Values by Interaction (Treatment $\times$ Extrinsic / Intrinsic Motivations) 


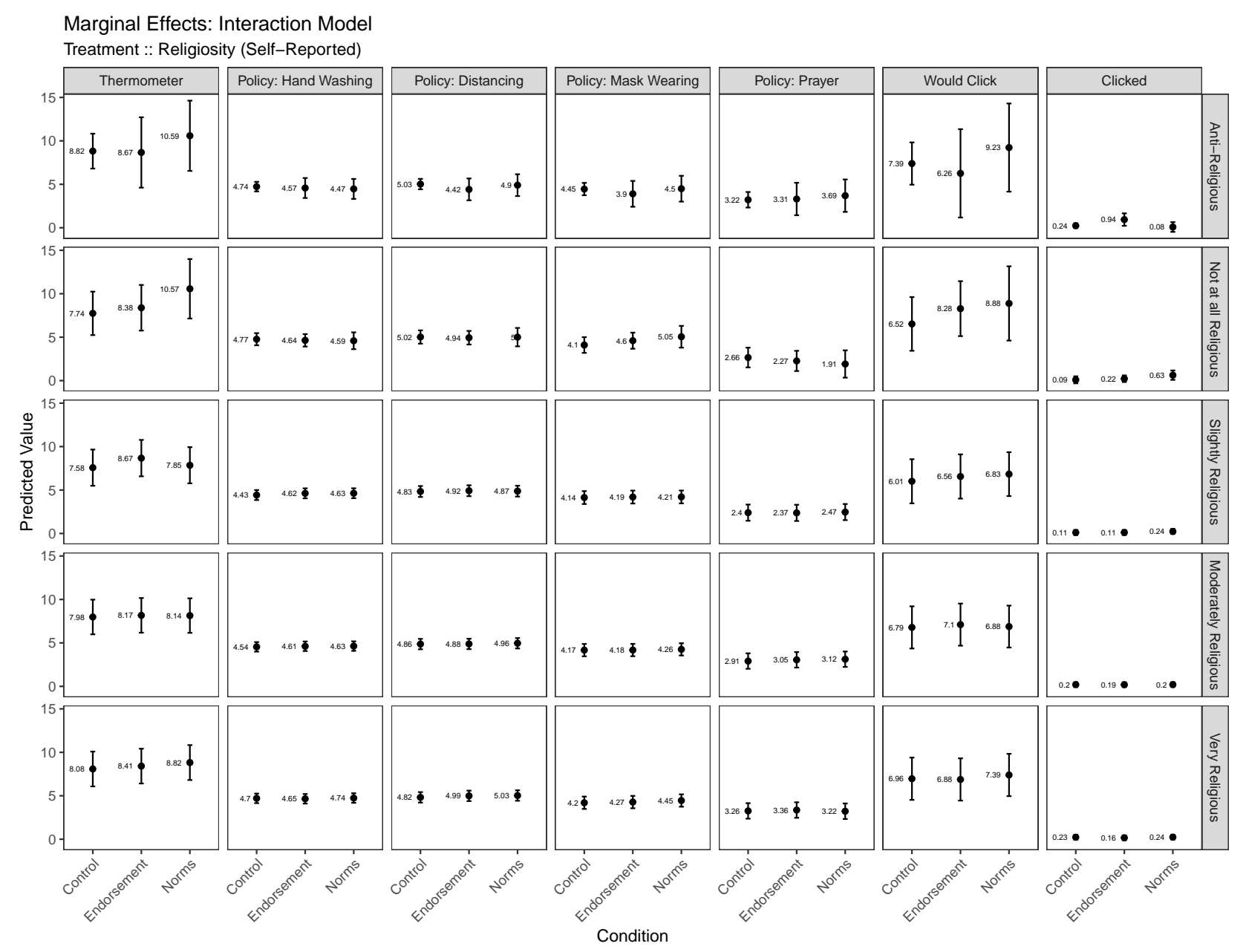

Figure G9: Predicted Values by Interaction (Treatment $\times$ Religiosity (Self-Reported)) 


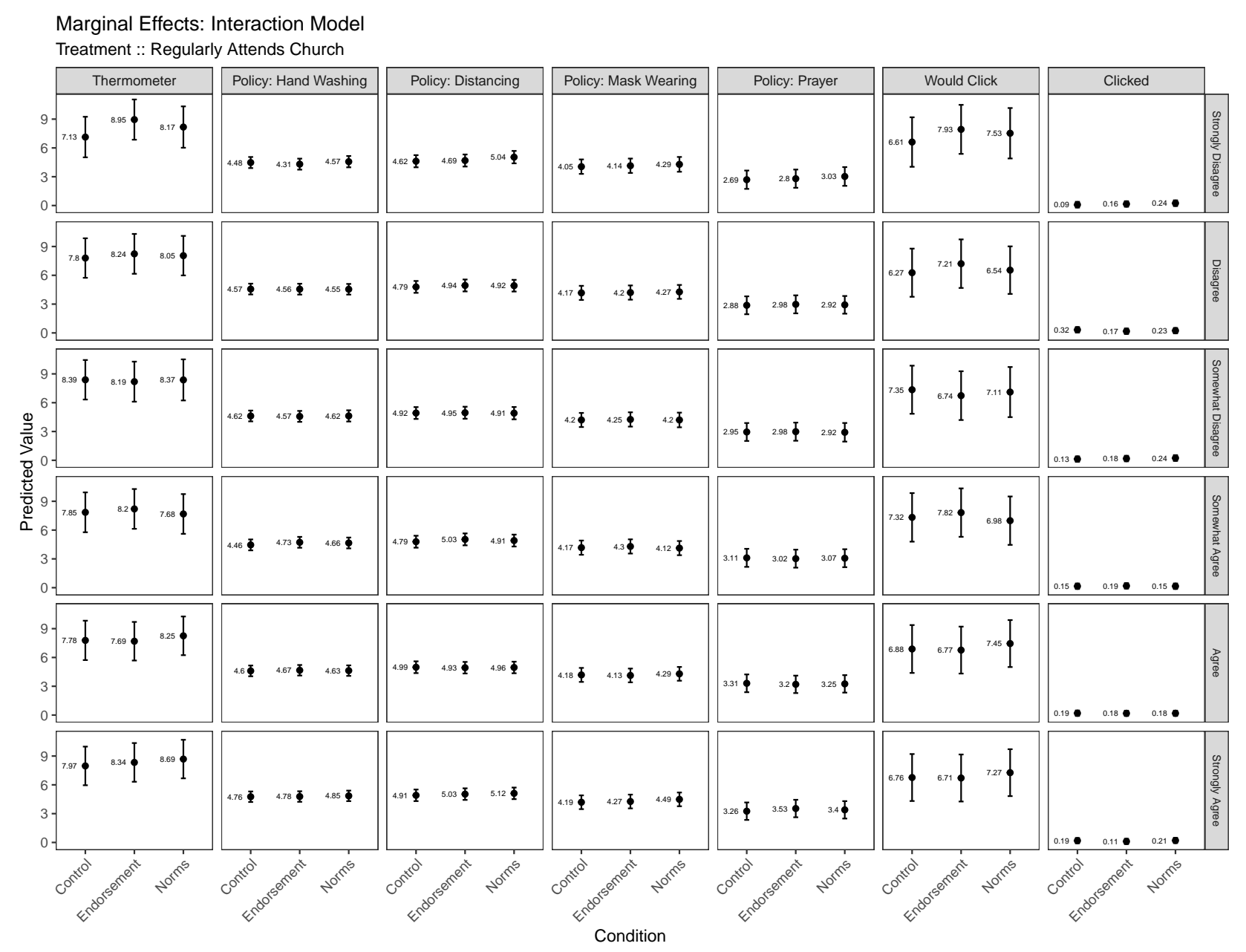

Figure G10: Predicted Values by Interaction (Treatment $\times$ Religiosity Index) 


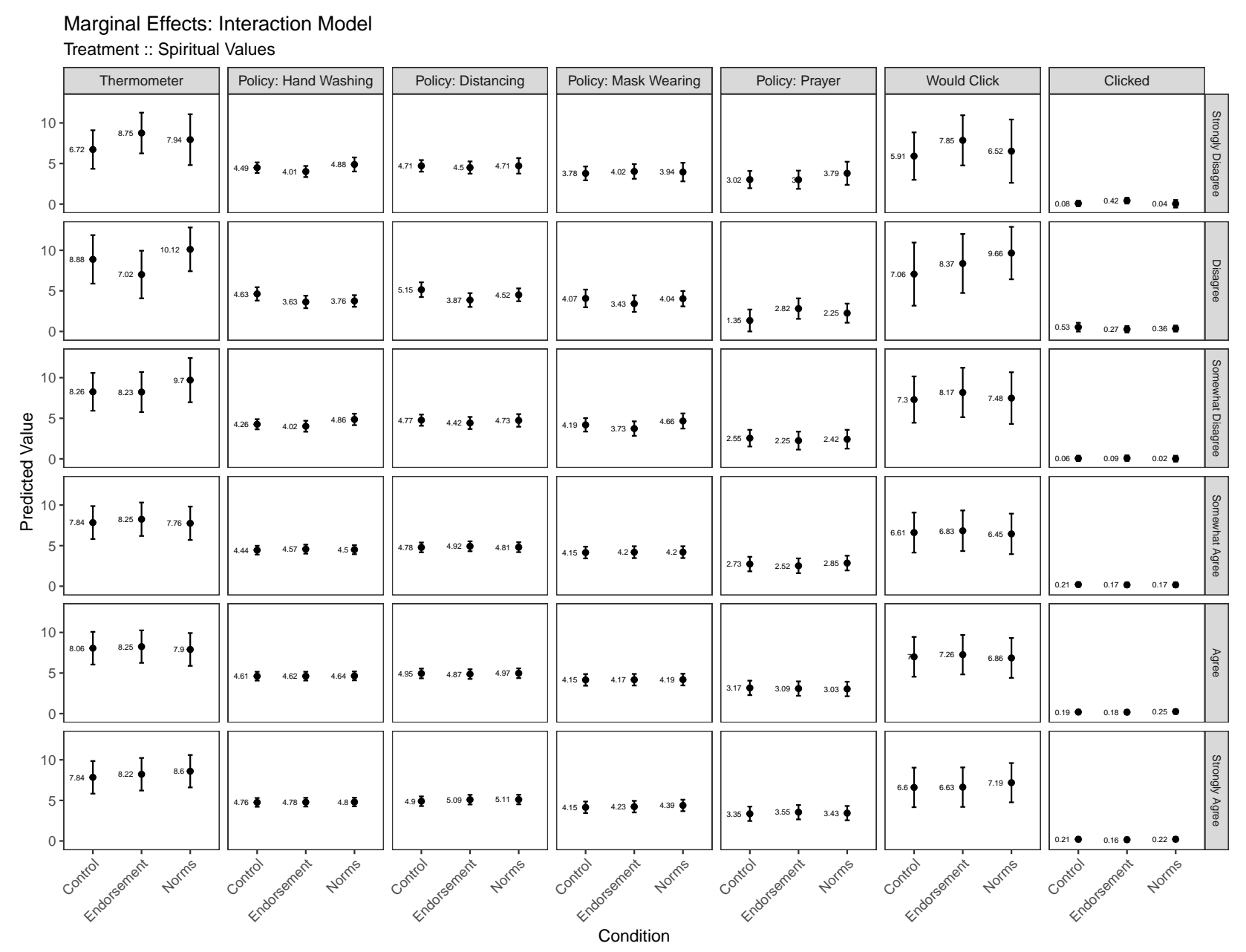

Figure G11: Predicted Values by Interaction (Treatment $\times$ Religiosity Index) 


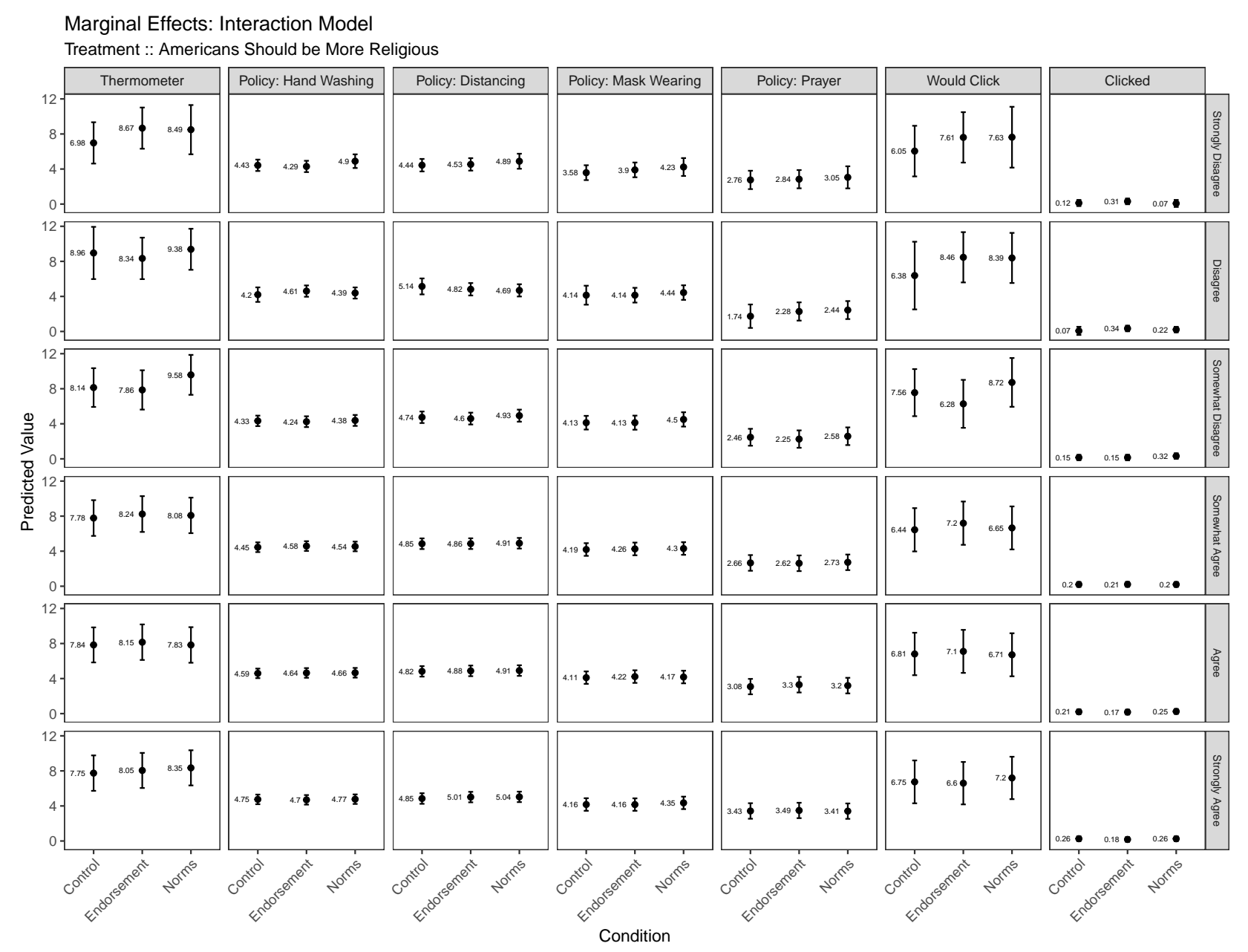

Figure G12: Predicted Values by Interaction (Treatment $\times$ Religiosity Index) 


\begin{tabular}{|c|c|c|c|c|c|c|c|}
\hline & Thermometer & Policy: Hand Washing & Policy: Distancing & Policy: Mask Wearing & Policy: Prayer & Would Click & Clicked \\
\hline Intercept & $\begin{array}{c}1.558 \\
(1.136)\end{array}$ & $\begin{array}{c}3.736^{* * *} \\
(0.312)\end{array}$ & $\begin{array}{c}3.512^{* * *} \\
(0.341)\end{array}$ & $\begin{array}{c}1.960^{* * *} \\
(0.403)\end{array}$ & $\begin{array}{c}2.577^{* * *} \\
(0.506)\end{array}$ & $\begin{array}{l}-1.100 \\
(1.380)\end{array}$ & $\begin{array}{c}0.067 \\
(0.161)\end{array}$ \\
\hline Endorsement $\times$ Anti-Religious & $\begin{array}{l}-0.155 \\
(1.793)\end{array}$ & $\begin{array}{l}-0.163 \\
(0.515)\end{array}$ & $\begin{array}{l}-0.612 \\
(0.563)\end{array}$ & $\begin{array}{l}-0.548 \\
(0.666)\end{array}$ & $\begin{array}{c}0.091 \\
(0.836)\end{array}$ & $\begin{array}{l}-1.130 \\
(2.274)\end{array}$ & $\begin{array}{c}0.704^{* *} \\
(0.341)\end{array}$ \\
\hline Norms $\times$ Anti-Religious & $\begin{array}{c}1.766 \\
(1.808)\end{array}$ & $\begin{array}{l}-0.265 \\
(0.519)\end{array}$ & $\begin{array}{l}-0.124 \\
(0.567)\end{array}$ & $\begin{array}{c}0.043 \\
(0.671)\end{array}$ & $\begin{array}{c}0.472 \\
(0.843)\end{array}$ & $\begin{array}{l}1.837 \\
(2.295)\end{array}$ & $\begin{array}{l}-0.153 \\
(0.252)\end{array}$ \\
\hline Control $\times$ Not at all Religious & $\begin{array}{l}-1.080 \\
(0.795)\end{array}$ & $\begin{array}{c}0.029 \\
(0.228)\end{array}$ & $\begin{array}{l}-0.007 \\
(0.249)\end{array}$ & $\begin{array}{l}-0.355 \\
(0.295)\end{array}$ & $\begin{array}{l}-0.565 \\
(0.370)\end{array}$ & $\begin{array}{l}-0.869 \\
(1.005)\end{array}$ & $\begin{array}{l}-0.145 \\
(0.154)\end{array}$ \\
\hline Endorsement $\times$ Not at all Religious & $\begin{array}{l}-0.437 \\
(0.898)\end{array}$ & $\begin{array}{l}-0.100 \\
(0.243)\end{array}$ & $\begin{array}{l}-0.090 \\
(0.265)\end{array}$ & $\begin{array}{l}0.144 \\
(0.314)\end{array}$ & $\begin{array}{c}-0.955^{* *} \\
(0.394)\end{array}$ & $\begin{array}{c}0.892 \\
(1.072)\end{array}$ & $\begin{array}{l}-0.018 \\
(0.140)\end{array}$ \\
\hline Norms $\times$ Not at all Religious & $\begin{array}{c}1.748 \\
(1.427)\end{array}$ & $\begin{array}{l}-0.148 \\
(0.410)\end{array}$ & $\begin{array}{l}-0.023 \\
(0.448)\end{array}$ & $\begin{array}{c}0.597 \\
(0.530)\end{array}$ & $\begin{array}{c}-1.308^{* *} \\
(0.665)\end{array}$ & $\begin{array}{c}1.491 \\
(1.807)\end{array}$ & $\begin{array}{c}0.390 \\
(0.241)\end{array}$ \\
\hline Control $\times$ Slightly Religious & $\begin{array}{c}-1.246^{* * * *} \\
(0.369)\end{array}$ & $\begin{array}{c}-0.310^{* * * *} \\
(0.103)\end{array}$ & $\begin{array}{l}-0.194^{*} \\
(0.113)\end{array}$ & $\begin{array}{c}-0.317^{* *} \\
(0.134)\end{array}$ & $\begin{array}{c}-0.824 * * * \\
(0.168)\end{array}$ & $\begin{array}{c}-1.383^{* * *} \\
(0.459)\end{array}$ & $\begin{array}{c}-0.127^{* *} \\
(0.061)\end{array}$ \\
\hline Endorsement $\times$ Slightly Religious & $\begin{array}{l}-0.148 \\
(0.375)\end{array}$ & $\begin{array}{l}-0.116 \\
(0.105)\end{array}$ & $\begin{array}{l}-0.105 \\
(0.114)\end{array}$ & $\begin{array}{c}-0.258^{*} \\
(0.135)\end{array}$ & $\begin{array}{c}-0.847^{* * *} \\
(0.170)\end{array}$ & $\begin{array}{c}-0.831^{*} \\
(0.465)\end{array}$ & $\begin{array}{c}-0.124^{* *} \\
(0.059)\end{array}$ \\
\hline Norms $\times$ Slightly Religious & $\begin{array}{c}-0.970^{* * *} \\
(0.364)\end{array}$ & $\begin{array}{l}-0.105 \\
(0.100)\end{array}$ & $\begin{array}{l}-0.153 \\
(0.109)\end{array}$ & $\begin{array}{c}-0.246^{*} \\
(0.129)\end{array}$ & $\begin{array}{c}-0.755^{* * *} \\
(0.162)\end{array}$ & $\begin{array}{l}-0.560 \\
(0.449)\end{array}$ & $\begin{array}{c}-0.002 \\
(0.057)\end{array}$ \\
\hline Control $\times$ Moderately Religious & $\begin{array}{c}-0.839^{* * *} * \\
(0.228)\end{array}$ & $\begin{array}{c}-0.193^{* * *} \\
(0.064)\end{array}$ & $\begin{array}{c}-0.163^{* *} \\
(0.070)\end{array}$ & $\begin{array}{c}-0.284^{* * *} * \\
(0.082)\end{array}$ & $\begin{array}{c}-0.312^{* * *} \\
(0.103)\end{array}$ & $\begin{array}{c}-0.607^{* *} \\
(0.283)\end{array}$ & $\begin{array}{l}-0.039 \\
(0.036)\end{array}$ \\
\hline Endorsement $\times$ Moderately Religious & $\begin{array}{c}-0.652^{* * *} \\
(0.221)\end{array}$ & $\begin{array}{c}-0.121^{* *} \\
(0.062)\end{array}$ & $\begin{array}{c}-0.146^{* *} \\
(0.067)\end{array}$ & $\begin{array}{c}-0.276^{* * *} \\
(0.080)\end{array}$ & $\begin{array}{c}-0.169^{*} \\
(0.100)\end{array}$ & $\begin{array}{c}-0.288 \\
(0.273)\end{array}$ & $\begin{array}{l}-0.046 \\
(0.035)\end{array}$ \\
\hline Norms $\times$ Moderately Religious & $\begin{array}{c}-0.678^{* * *} \\
(0.226)\end{array}$ & $\begin{array}{c}-0.104^{*} \\
(0.063)\end{array}$ & $\begin{array}{l}-0.069 \\
(0.069)\end{array}$ & $\begin{array}{c}-0.198^{* *} \\
(0.081)\end{array}$ & $\begin{array}{l}-0.098 \\
(0.102)\end{array}$ & $\begin{array}{c}-0.511^{*} \\
(0.280)\end{array}$ & $\begin{array}{l}-0.036 \\
(0.036)\end{array}$ \\
\hline Control $\times$ Very Religious & $\begin{array}{c}-0.740^{* * * *} \\
(0.219)\end{array}$ & $\begin{array}{l}-0.033 \\
(0.061)\end{array}$ & $\begin{array}{c}-0.209^{* * * *} \\
(0.066)\end{array}$ & $\begin{array}{c}-0.258^{* * * *} \\
(0.079)\end{array}$ & $\begin{array}{l}0.036 \\
(0.099)\end{array}$ & $\begin{array}{l}-0.431 \\
(0.271)\end{array}$ & $\begin{array}{l}-0.007 \\
(0.036)\end{array}$ \\
\hline Endorsement $\times$ Very Religious & $\begin{array}{r}-0.407^{*} \\
(0.226)\end{array}$ & $\begin{array}{l}-0.084 \\
(0.063)\end{array}$ & $\begin{array}{l}-0.038 \\
(0.069)\end{array}$ & $\begin{array}{c}-0.179^{* *} \\
(0.081)\end{array}$ & $\begin{array}{l}0.137 \\
(0.102)\end{array}$ & $\begin{array}{l}-0.516^{*} \\
(0.279)\end{array}$ & $\begin{array}{r}-0.074^{* *} \\
(0.036)\end{array}$ \\
\hline Observations & 1682 & 1774 & 1775 & 1775 & 1774 & 1739 & 1213 \\
\hline$R^{2}$ & 0.352 & 0.101 & 0.194 & 0.288 & 0.128 & 0.332 & 0.062 \\
\hline$F$ & 19.742 & 4.314 & 9.263 & 15.522 & 5.624 & 18.680 & 1.707 \\
\hline $\begin{array}{l}\text { Note: Models include all controls. } \\
{ }^{*} p<0.1,{ }^{* *} p<0.05,{ }^{* * *} p<0.01\end{array}$ & & & & & & & \\
\hline
\end{tabular}

Table G22: Interaction Model: Treatment $\times$ Religiosity (Self-Reported) 


\begin{tabular}{|c|c|c|c|c|c|c|c|}
\hline & Thermometer & Policy: Hand Washing & Policy: Distancing & Policy: Mask Wearing & Policy: Prayer & Would Click & Clicked \\
\hline Social Identity $\times$ Norms & $\begin{array}{c}0.573^{* *} \\
(0.228)\end{array}$ & $\begin{array}{l}-0.019 \\
(0.063)\end{array}$ & $\begin{array}{l}0.122^{*} \\
(0.069)\end{array}$ & $\begin{array}{c}0.197^{* *} \\
(0.082)\end{array}$ & $\begin{array}{c}0.108 \\
(0.106)\end{array}$ & $\begin{array}{l}0.496^{*} \\
(0.285)\end{array}$ & $\begin{array}{c}0.008 \\
(0.039)\end{array}$ \\
\hline Social Identity $\times$ Endorsement & $\begin{array}{c}0.556^{* *} \\
(0.233)\end{array}$ & $\begin{array}{c}0.002 \\
(0.065)\end{array}$ & $\begin{array}{l}0.131^{*} \\
(0.072)\end{array}$ & $\begin{array}{c}0.119 \\
(0.085)\end{array}$ & $\begin{array}{c}0.233^{* *} \\
(0.110)\end{array}$ & $\begin{array}{c}0.139 \\
(0.294)\end{array}$ & $\begin{array}{l}-0.058 \\
(0.040)\end{array}$ \\
\hline Insurance $\times$ Norms & $\begin{array}{c}0.443 \\
(0.505)\end{array}$ & $\begin{array}{c}0.212 \\
(0.138)\end{array}$ & $\begin{array}{l}0.286^{*} \\
(0.152)\end{array}$ & $\begin{array}{c}0.196 \\
(0.181)\end{array}$ & $\begin{array}{c}0.053 \\
(0.234)\end{array}$ & $\begin{array}{l}1.072^{*} \\
(0.632)\end{array}$ & $\begin{array}{c}0.132 \\
(0.082)\end{array}$ \\
\hline Insurance $\times$ Endorsement & $\begin{array}{l}-0.044 \\
(0.519)\end{array}$ & $\begin{array}{c}0.172 \\
(0.140)\end{array}$ & $\begin{array}{l}0.188 \\
(0.154)\end{array}$ & $\begin{array}{l}-0.019 \\
(0.184)\end{array}$ & $\begin{array}{l}0.221 \\
(0.237)\end{array}$ & $\begin{array}{c}0.600 \\
(0.641)\end{array}$ & $\begin{array}{l}0.044 \\
(0.084)\end{array}$ \\
\hline Morality $\times$ Norms & $\begin{array}{c}0.636 \\
(0.407)\end{array}$ & $\begin{array}{c}0.129 \\
(0.112)\end{array}$ & $\begin{array}{c}0.170 \\
(0.123)\end{array}$ & $\begin{array}{c}0.116 \\
(0.146)\end{array}$ & $\begin{array}{l}-0.173 \\
(0.188)\end{array}$ & $\begin{array}{l}-0.069 \\
(0.507)\end{array}$ & $\begin{array}{l}-0.098 \\
(0.064)\end{array}$ \\
\hline Morality $\times$ Endorsement & $\begin{array}{c}0.385 \\
(0.433)\end{array}$ & $\begin{array}{l}-0.039 \\
(0.119)\end{array}$ & $\begin{array}{c}0.149 \\
(0.130)\end{array}$ & $\begin{array}{c}0.132 \\
(0.155)\end{array}$ & $\begin{array}{l}-0.083 \\
(0.200)\end{array}$ & $\begin{array}{c}0.137 \\
(0.538)\end{array}$ & $\begin{array}{c}-0.146^{* *} \\
(0.066)\end{array}$ \\
\hline Social Capital $\times$ Norms & $\begin{array}{c}0.126 \\
(0.579)\end{array}$ & $\begin{array}{c}0.139 \\
(0.158)\end{array}$ & $\begin{array}{c}0.006 \\
(0.174)\end{array}$ & $\begin{array}{c}0.158 \\
(0.207)\end{array}$ & $\begin{array}{c}0.127 \\
(0.267)\end{array}$ & $\begin{array}{l}-0.395 \\
(0.714)\end{array}$ & $\begin{array}{c}0.280^{* * *} \\
(0.097)\end{array}$ \\
\hline Social Capital $\times$ Endorsement & $\begin{array}{c}0.630 \\
(0.514)\end{array}$ & $\begin{array}{l}0.048 \\
(0.141)\end{array}$ & $\begin{array}{l}-0.009 \\
(0.155)\end{array}$ & $\begin{array}{c}0.018 \\
(0.185)\end{array}$ & $\begin{array}{c}0.079 \\
(0.238)\end{array}$ & $\begin{array}{c}0.217 \\
(0.640)\end{array}$ & $\begin{array}{c}0.070 \\
(0.081)\end{array}$ \\
\hline Heuristics $\times$ Norms & $\begin{array}{c}0.537 \\
(0.387)\end{array}$ & $\begin{array}{l}0.194^{*} \\
(0.106)\end{array}$ & $\begin{array}{c}0.244^{* *} \\
(0.117)\end{array}$ & $\begin{array}{l}0.240^{*} \\
(0.139)\end{array}$ & $\begin{array}{c}0.082 \\
(0.180)\end{array}$ & $\begin{array}{c}0.639 \\
(0.483)\end{array}$ & $\begin{array}{l}-0.012 \\
(0.066)\end{array}$ \\
\hline Heuristics $\times$ Endorsement & $\begin{array}{c}0.261 \\
(0.370)\end{array}$ & $\begin{array}{l}0.068 \\
(0.102)\end{array}$ & $\begin{array}{c}0.061 \\
(0.112)\end{array}$ & $\begin{array}{c}0.011 \\
(0.133)\end{array}$ & $\begin{array}{c}0.063 \\
(0.172)\end{array}$ & $\begin{array}{c}0.422 \\
(0.462)\end{array}$ & $\begin{array}{c}0.010 \\
(0.065)\end{array}$ \\
\hline Tradition $\times$ Norms & $\begin{array}{c}0.091 \\
(0.432)\end{array}$ & $\begin{array}{l}-0.006 \\
(0.121)\end{array}$ & $\begin{array}{c}0.061 \\
(0.133)\end{array}$ & $\begin{array}{c}0.116 \\
(0.159)\end{array}$ & $\begin{array}{c}0.184 \\
(0.205)\end{array}$ & $\begin{array}{l}-0.152 \\
(0.549)\end{array}$ & $\begin{array}{c}0.048 \\
(0.070)\end{array}$ \\
\hline Tradition $\times$ Endorsement & $\begin{array}{c}0.045 \\
(0.433)\end{array}$ & $\begin{array}{l}0.150 \\
(0.120)\end{array}$ & $\begin{array}{c}0.083 \\
(0.133)\end{array}$ & $\begin{array}{l}-0.018 \\
(0.158)\end{array}$ & $\begin{array}{l}-0.143 \\
(0.204)\end{array}$ & $\begin{array}{l}-0.043 \\
(0.544)\end{array}$ & $\begin{array}{c}0.012 \\
(0.070)\end{array}$ \\
\hline Observations & 1604 & 1687 & 1688 & 1688 & 1688 & 1655 & 1152 \\
\hline$R^{2}$ & 0.360 & 0.100 & 0.203 & 0.298 & 0.086 & 0.337 & 0.070 \\
\hline$F$ & 17.827 & 3.719 & 8.492 & 14.209 & 3.149 & 16.644 & 1.684 \\
\hline
\end{tabular}

Note: Each row presents coefficients from a model with all controls, in which the omitted category is the control condition for the relevant religion motivation respondents.

For example, for the first two rows, the omitted category is the control condition for respondents whose main religion motivation is social identity. For the next two rows,

the omitted cateogry is the control condition for respondents whose main religion motivation is insurance. Therefore, each pair of rows reports results from separate regressions. See Tables F24-F29 for full models

${ }^{*} p<0.1,{ }^{* *} p<0.05, * * * p<0.01$

Table G23: Interaction Model: Treatment $\times$ Religious Motivations 


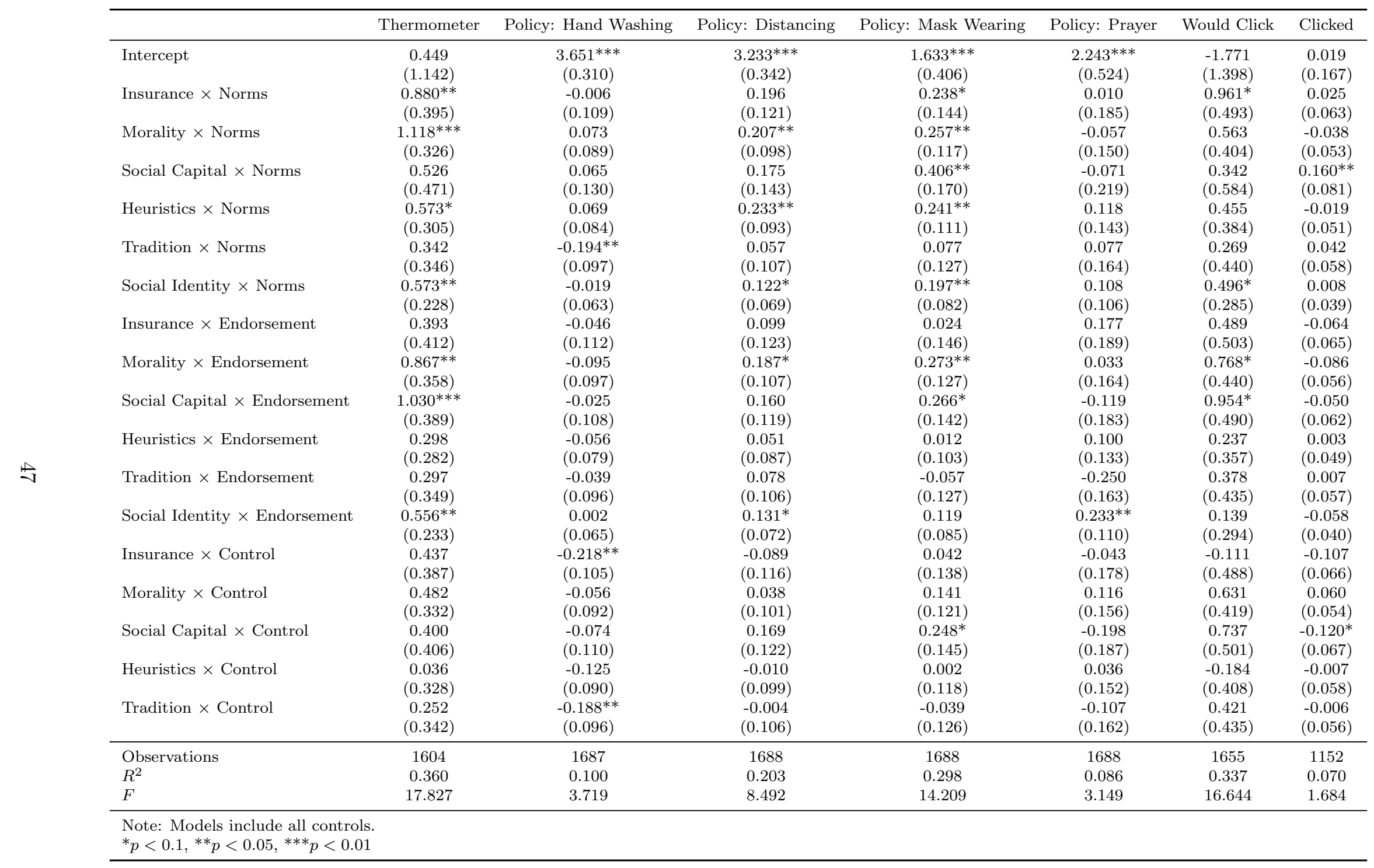

Table G24: Interaction Model: Treatment $\times$ Religious Motivations

Omitted Category $=$ Social Identity $\times$ Control 


\begin{tabular}{|c|c|c|c|c|c|c|c|c|}
\hline & & Thermometer & Policy: Hand Washing & Policy: Distancing & Policy: Mask Wearing & Policy: Prayer & Would Click & Clicked \\
\hline & Intercept & $\begin{array}{c}0.886 \\
(1.190)\end{array}$ & $\begin{array}{c}3.433^{* * *} \\
(0.321)\end{array}$ & $\begin{array}{c}3.143^{* * *} \\
(0.355)\end{array}$ & $\begin{array}{c}1.675^{* * *} \\
(0.422)\end{array}$ & $\begin{array}{c}2.200^{* * *} \\
(0.544)\end{array}$ & $\begin{array}{c}-1.882 \\
(1.456)\end{array}$ & $\begin{array}{c}-0.088 \\
(0.176)\end{array}$ \\
\hline & Insurance $\times$ Norms & 0.443 & 0.212 & $0.286^{*}$ & 0.196 & 0.053 & $1.072^{*}$ & 0.132 \\
\hline & & $(0.505)$ & $(0.138)$ & $(0.152)$ & $(0.181)$ & $(0.234)$ & $(0.632)$ & $(0.082)$ \\
\hline & Morality $\times$ Norms & 0.682 & $0.291^{* *}$ & $0.297^{* *}$ & 0.215 & -0.013 & 0.674 & 0.069 \\
\hline & & $(0.454)$ & $(0.123)$ & $(0.135)$ & $(0.161)$ & $(0.208)$ & $(0.567)$ & $(0.075)$ \\
\hline & Social Capital $\times$ Norms & 0.090 & $0.283^{*}$ & 0.264 & $0.364^{*}$ & -0.028 & 0.453 & $0.268^{* * *}$ \\
\hline & & $(0.565)$ & $(0.154)$ & $(0.170)$ & $(0.202)$ & $(0.261)$ & $(0.703)$ & $(0.096)$ \\
\hline & Heuristics $\times$ Norms & 0.137 & $0.287^{* *}$ & $0.323^{* *}$ & 0.199 & 0.162 & 0.565 & 0.088 \\
\hline & & $(0.436)$ & $(0.119)$ & $(0.131)$ & $(0.156)$ & $(0.201)$ & $(0.551)$ & $(0.074)$ \\
\hline & Tradition $\times$ Norms & -0.094 & 0.024 & 0.146 & 0.035 & 0.120 & 0.380 & $0.150^{*}$ \\
\hline & & $(0.469)$ & $(0.129)$ & $(0.142)$ & $(0.169)$ & $(0.218)$ & $(0.593)$ & $(0.078)$ \\
\hline & Social Identity $\times$ Norms & 0.137 & $0.199^{*}$ & $0.212^{*}$ & 0.155 & 0.152 & 0.606 & $0.115^{*}$ \\
\hline & & $(0.391)$ & $(0.106)$ & $(0.116)$ & $(0.139)$ & $(0.179)$ & $(0.491)$ & $(0.066)$ \\
\hline & Insurance $\times$ Endorsement & -0.044 & 0.172 & 0.188 & -0.019 & 0.221 & 0.600 & 0.044 \\
\hline & & $(0.519)$ & $(0.140)$ & $(0.154)$ & $(0.184)$ & $(0.237)$ & $(0.641)$ & $(0.084)$ \\
\hline & Morality $\times$ Endorsement & 0.430 & 0.123 & $0.276^{*}$ & 0.231 & 0.077 & 0.879 & 0.021 \\
\hline & & $(0.478)$ & $(0.129)$ & $(0.142)$ & $(0.169)$ & $(0.218)$ & $(0.594)$ & $(0.077)$ \\
\hline & Social Capital $\times$ Endorsement & 0.593 & 0.193 & $0.250^{*}$ & 0.224 & -0.076 & $1.065^{*}$ & 0.057 \\
\hline & & $(0.500)$ & $(0.137)$ & $(0.151)$ & $(0.179)$ & $(0.232)$ & $(0.631)$ & $(0.082)$ \\
\hline & Heuristics $\times$ Endorsement & -0.139 & 0.162 & 0.140 & -0.030 & 0.143 & 0.348 & 0.111 \\
\hline \multirow{19}{*}{$\overleftrightarrow{\infty}$} & & $(0.423)$ & $(0.115)$ & $(0.127)$ & $(0.151)$ & $(0.195)$ & $(0.535)$ & $(0.072)$ \\
\hline & Tradition $\times$ Endorsement & -0.139 & 0.179 & 0.168 & -0.099 & -0.207 & 0.489 & 0.114 \\
\hline & & $(0.470)$ & $(0.128)$ & $(0.141)$ & $(0.168)$ & $(0.217)$ & $(0.589)$ & $(0.078)$ \\
\hline & Social Identity $\times$ Endorsement & 0.120 & $0.220^{* *}$ & $0.221^{*}$ & 0.077 & 0.276 & 0.250 & 0.049 \\
\hline & & $(0.393)$ & $(0.106)$ & $(0.117)$ & $(0.140)$ & $(0.180)$ & $(0.495)$ & $(0.067)$ \\
\hline & Morality $\times$ Control & 0.045 & 0.162 & 0.127 & 0.099 & 0.160 & 0.742 & $0.167^{* *}$ \\
\hline & & $(0.458)$ & $(0.125)$ & $(0.138)$ & $(0.164)$ & $(0.212)$ & $(0.578)$ & $(0.076)$ \\
\hline & Social Capital $\times$ Control & -0.037 & 0.144 & $0.259^{*}$ & 0.206 & -0.155 & 0.848 & -0.013 \\
\hline & & $(0.513)$ & $(0.139)$ & $(0.153)$ & $(0.182)$ & $(0.235)$ & $(0.639)$ & $(0.085)$ \\
\hline & Heuristics $\times$ Control & -0.400 & 0.093 & 0.079 & -0.040 & 0.080 & -0.074 & 0.100 \\
\hline & & $(0.456)$ & $(0.123)$ & $(0.136)$ & $(0.162)$ & $(0.208)$ & $(0.570)$ & $(0.079)$ \\
\hline & Tradition $\times$ Control & -0.185 & 0.030 & 0.085 & -0.081 & -0.064 & 0.531 & 0.102 \\
\hline & & $(0.466)$ & $(0.128)$ & $(0.141)$ & $(0.168)$ & $(0.216)$ & $(0.590)$ & $(0.078)$ \\
\hline & Social Identity $\times$ Control & -0.437 & $0.218^{* *}$ & 0.089 & -0.042 & 0.043 & 0.111 & 0.107 \\
\hline & & $(0.387)$ & $(0.105)$ & $(0.116)$ & $(0.138)$ & $(0.178)$ & $(0.488)$ & $(0.066)$ \\
\hline & Observations & 1604 & 1687 & 1688 & 1688 & 1688 & 1655 & 1152 \\
\hline & $R^{2}$ & 0.360 & 0.100 & 0.203 & 0.298 & 0.086 & 0.337 & 0.070 \\
\hline & $F$ & 17.827 & 3.719 & 8.492 & 14.209 & 3.149 & 16.644 & 1.684 \\
\hline & $\begin{array}{l}\text { Note: Models include all controls. } \\
{ }^{*} p<0.1,{ }^{* *} p<0.05,{ }^{* * *} p<0.01\end{array}$ & & & & & & & \\
\hline
\end{tabular}

Table G25: Interaction Model: Treatment $\times$ Religious Motivations Omitted Category $=$ Insurance $\times$ Control 


\begin{tabular}{|c|c|c|c|c|c|c|c|}
\hline & Thermometer & Policy: Hand Washing & Policy: Distancing & Policy: Mask Wearing & Policy: Prayer & Would Click & Clicked \\
\hline Intercept & $\begin{array}{c}0.931 \\
(1.177)\end{array}$ & $\begin{array}{c}3.594^{* * *} \\
(0.320)\end{array}$ & $\begin{array}{c}3.270^{* * *} \\
(0.352)\end{array}$ & $\begin{array}{c}1.775^{* * *} \\
(0.419)\end{array}$ & $\begin{array}{c}2.359 * * * \\
(0.541)\end{array}$ & $\begin{array}{l}-1.139 \\
(1.443)\end{array}$ & $\begin{array}{c}0.079 \\
(0.171)\end{array}$ \\
\hline Insurance $\times$ Norms & $\begin{array}{l}0.398 \\
(0.464)\end{array}$ & $\begin{array}{c}0.051 \\
(0.129)\end{array}$ & $\begin{array}{c}0.159 \\
(0.142)\end{array}$ & $\begin{array}{c}0.097 \\
(0.169)\end{array}$ & $\begin{array}{l}-0.106 \\
(0.217)\end{array}$ & $\begin{array}{l}0.330 \\
(0.581)\end{array}$ & $\begin{array}{l}-0.035 \\
(0.073)\end{array}$ \\
\hline Morality $\times$ Norms & $\begin{array}{l}0.636 \\
(0.407)\end{array}$ & $\begin{array}{c}0.129 \\
(0.112)\end{array}$ & $\begin{array}{l}0.170 \\
(0.123)\end{array}$ & $\begin{array}{l}0.116 \\
(0.146)\end{array}$ & $\begin{array}{l}-0.173 \\
(0.188)\end{array}$ & $\begin{array}{l}-0.069 \\
(0.507)\end{array}$ & $\begin{array}{l}-0.098 \\
(0.064)\end{array}$ \\
\hline Social Capital $\times$ Norms & $\begin{array}{c}0.044 \\
(0.530)\end{array}$ & $\begin{array}{c}0.121 \\
(0.146)\end{array}$ & $\begin{array}{c}0.137 \\
(0.161)\end{array}$ & $\begin{array}{c}0.265 \\
(0.191)\end{array}$ & $\begin{array}{l}-0.188 \\
(0.247)\end{array}$ & $\begin{array}{l}-0.289 \\
(0.659)\end{array}$ & $\begin{array}{c}0.100 \\
(0.089)\end{array}$ \\
\hline Heuristics $\times$ Norms & $\begin{array}{c}0.091 \\
(0.391)\end{array}$ & $\begin{array}{l}0.125 \\
(0.109)\end{array}$ & $\begin{array}{l}0.196 \\
(0.119)\end{array}$ & $\begin{array}{l}0.100 \\
(0.142)\end{array}$ & $\begin{array}{c}0.002 \\
(0.183)\end{array}$ & $\begin{array}{l}-0.177 \\
(0.493)\end{array}$ & $\begin{array}{l}-0.079 \\
(0.063)\end{array}$ \\
\hline Tradition $\times$ Norms & $\begin{array}{l}-0.139 \\
(0.422)\end{array}$ & $\begin{array}{l}-0.138 \\
(0.118)\end{array}$ & $\begin{array}{l}0.019 \\
(0.130)\end{array}$ & $\begin{array}{l}-0.064 \\
(0.155)\end{array}$ & $\begin{array}{l}-0.039 \\
(0.199)\end{array}$ & $\begin{array}{l}-0.362 \\
(0.535)\end{array}$ & $\begin{array}{l}-0.018 \\
(0.068)\end{array}$ \\
\hline Social Identity $\times$ Norms & $\begin{array}{l}0.091 \\
(0.334)\end{array}$ & $\begin{array}{l}0.037 \\
(0.093)\end{array}$ & $\begin{array}{l}0.085 \\
(0.102)\end{array}$ & $\begin{array}{l}0.056 \\
(0.121)\end{array}$ & $\begin{array}{l}-0.008 \\
(0.156)\end{array}$ & $\begin{array}{l}-0.136 \\
(0.421)\end{array}$ & $\begin{array}{l}-0.052 \\
(0.053)\end{array}$ \\
\hline Insurance $\times$ Endorsement & $\begin{array}{l}-0.089 \\
(0.479)\end{array}$ & $\begin{array}{c}0.010 \\
(0.131)\end{array}$ & $\begin{array}{c}0.061 \\
(0.144)\end{array}$ & $\begin{array}{l}-0.118 \\
(0.171)\end{array}$ & $\begin{array}{c}0.061 \\
(0.221)\end{array}$ & $\begin{array}{l}-0.143 \\
(0.590)\end{array}$ & $\begin{array}{l}-0.124^{*} \\
(0.075)\end{array}$ \\
\hline Morality $\times$ Endorsement & $\begin{array}{l}0.385 \\
(0.433)\end{array}$ & $\begin{array}{l}-0.039 \\
(0.119)\end{array}$ & $\begin{array}{c}0.149 \\
(0.130)\end{array}$ & $\begin{array}{c}0.132 \\
(0.155)\end{array}$ & $\begin{array}{l}-0.083 \\
(0.200)\end{array}$ & $\begin{array}{c}0.137 \\
(0.538)\end{array}$ & $\begin{array}{r}-0.146^{* *} \\
(0.066)\end{array}$ \\
\hline Social Capital $\times$ Endorsement & $\begin{array}{l}0.548 \\
(0.457)\end{array}$ & $\begin{array}{l}0.031 \\
(0.127)\end{array}$ & $\begin{array}{l}0.123 \\
(0.140)\end{array}$ & $\begin{array}{l}0.125 \\
(0.166)\end{array}$ & $\begin{array}{l}-0.236 \\
(0.215)\end{array}$ & $\begin{array}{l}0.323 \\
(0.577)\end{array}$ & $\begin{array}{l}-0.110 \\
(0.072)\end{array}$ \\
\hline Tradition $\times$ Endorsement & $\begin{array}{l}-0.185 \\
(0.424)\end{array}$ & $\begin{array}{c}0.018 \\
(0.118)\end{array}$ & $\begin{array}{l}0.041 \\
(0.129)\end{array}$ & $\begin{array}{l}-0.199 \\
(0.154)\end{array}$ & $\begin{array}{l}-0.366^{*} \\
(0.199)\end{array}$ & $\begin{array}{l}-0.254 \\
(0.532)\end{array}$ & $\begin{array}{l}-0.053 \\
(0.068)\end{array}$ \\
\hline Social Identity $\times$ Endorsement & $\begin{array}{c}0.075 \\
(0.338)\end{array}$ & $\begin{array}{c}0.059 \\
(0.094)\end{array}$ & $\begin{array}{c}0.094 \\
(0.103)\end{array}$ & $\begin{array}{l}-0.022 \\
(0.123)\end{array}$ & $\begin{array}{c}0.117 \\
(0.159)\end{array}$ & $\begin{array}{l}-0.493 \\
(0.427)\end{array}$ & $\begin{array}{r}-0.118^{* *} \\
(0.054)\end{array}$ \\
\hline Insurance $\times$ Control & $\begin{array}{l}-0.045 \\
(0.458)\end{array}$ & $\begin{array}{l}-0.162 \\
(0.125)\end{array}$ & $\begin{array}{l}-0.127 \\
(0.138)\end{array}$ & $\begin{array}{l}-0.099 \\
(0.164)\end{array}$ & $\begin{array}{l}-0.160 \\
(0.212)\end{array}$ & $\begin{array}{l}-0.742 \\
(0.578)\end{array}$ & $\begin{array}{r}-0.167^{* *} \\
(0.076)\end{array}$ \\
\hline Social Capital $\times$ Control & $\begin{array}{l}-0.082 \\
(0.473)\end{array}$ & $\begin{array}{l}-0.018 \\
(0.130)\end{array}$ & $\begin{array}{c}0.132 \\
(0.143)\end{array}$ & $\begin{array}{c}0.107 \\
(0.170)\end{array}$ & $\begin{array}{l}-0.314 \\
(0.219)\end{array}$ & $\begin{array}{l}0.105 \\
(0.588)\end{array}$ & $\begin{array}{r}-0.180^{* *} \\
(0.076)\end{array}$ \\
\hline Heuristics $\times$ Control & $\begin{array}{l}-0.446 \\
(0.409)\end{array}$ & $\begin{array}{l}-0.068 \\
(0.113)\end{array}$ & $\begin{array}{l}-0.048 \\
(0.124)\end{array}$ & $\begin{array}{l}-0.140 \\
(0.147)\end{array}$ & $\begin{array}{l}-0.080 \\
(0.190)\end{array}$ & $\begin{array}{l}-0.816 \\
(0.511)\end{array}$ & $\begin{array}{l}-0.067 \\
(0.068)\end{array}$ \\
\hline Tradition $\times$ Control & $\begin{array}{l}-0.230 \\
(0.421)\end{array}$ & $\begin{array}{l}-0.132 \\
(0.118)\end{array}$ & $\begin{array}{l}-0.042 \\
(0.129)\end{array}$ & $\begin{array}{l}-0.181 \\
(0.154)\end{array}$ & $\begin{array}{l}-0.223 \\
(0.199)\end{array}$ & $\begin{array}{l}-0.211 \\
(0.534)\end{array}$ & $\begin{array}{l}-0.066 \\
(0.067)\end{array}$ \\
\hline Social Identity $\times$ Control & $\begin{array}{l}-0.482 \\
(0.332)\end{array}$ & $\begin{array}{c}0.056 \\
(0.092)\end{array}$ & $\begin{array}{l}-0.038 \\
(0.101)\end{array}$ & $\begin{array}{l}-0.141 \\
(0.121)\end{array}$ & $\begin{array}{l}-0.116 \\
(0.156)\end{array}$ & $\begin{array}{l}-0.631 \\
(0.419)\end{array}$ & $\begin{array}{l}-0.060 \\
(0.054)\end{array}$ \\
\hline Observations. & 1604 & 1687 & 1688 & 1688 & 1688 & 1655 & 1152 \\
\hline$R^{2}$ & 0.360 & 0.100 & 0.203 & 0.298 & 0.086 & 0.337 & 0.070 \\
\hline$F$ & 17.827 & 3.719 & 8.492 & 14.209 & 3.149 & 16.644 & 1.684 \\
\hline
\end{tabular}

Table G26: Interaction Model: Treatment $\times$ Religious Motivations

Omitted Category $=$ Morality $\times$ Control 


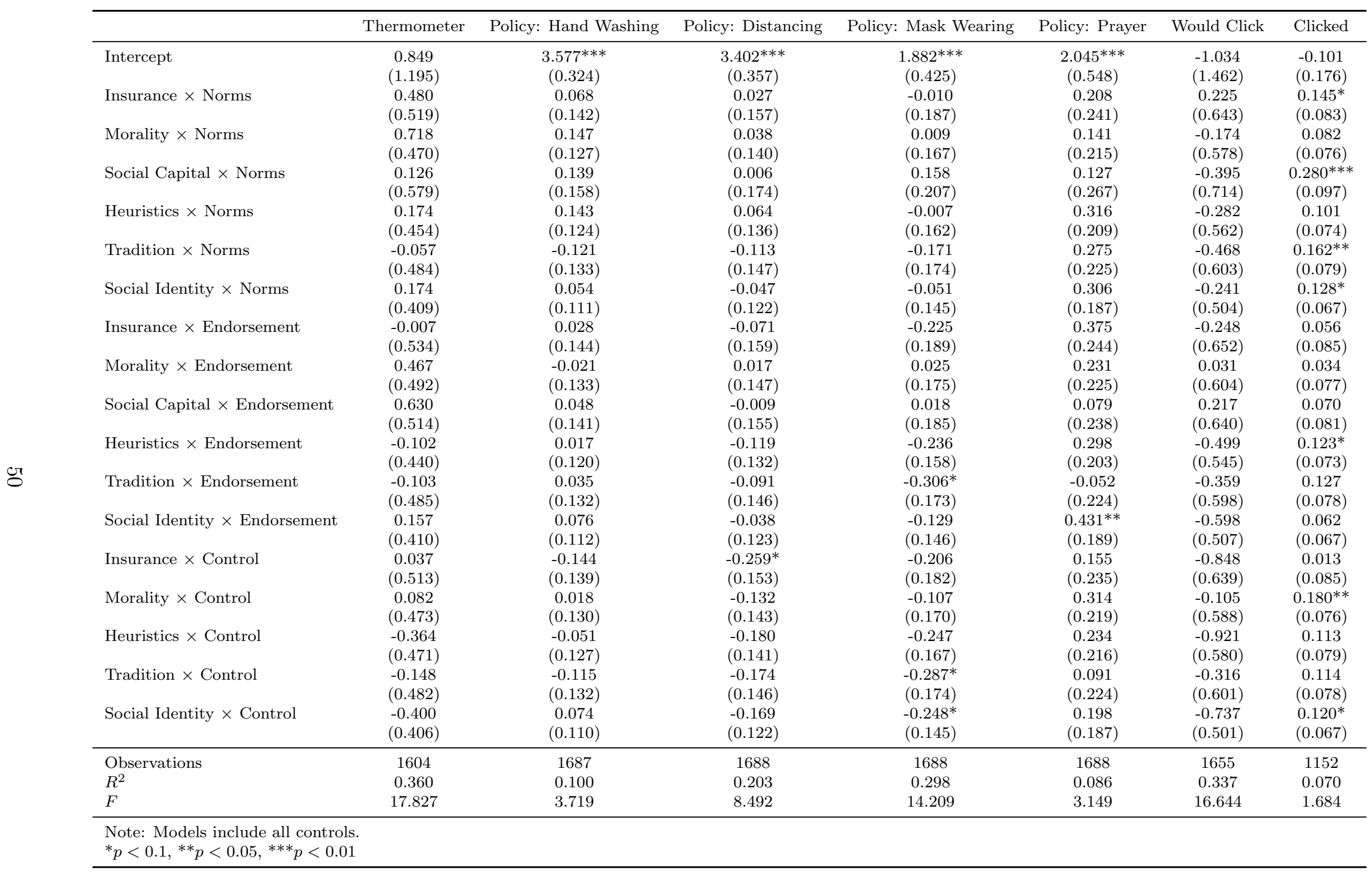

Table G27: Interaction Model: Treatment $\times$ Religious Motivations

Omitted Category $=$ Social Capital $\times$ Control 


\begin{tabular}{|c|c|c|c|c|c|c|c|}
\hline & Thermometer & Policy: Hand Washing & Policy: Distancing & Policy: Mask Wearing & Policy: Prayer & Would Click & Clicked \\
\hline Intercept & $\begin{array}{c}0.485 \\
(1.163)\end{array}$ & $\begin{array}{c}3.526^{* * *} \\
(0.315)\end{array}$ & $\begin{array}{c}3.222 * * * \\
(0.348)\end{array}$ & $\begin{array}{c}1.635^{* * *} \\
(0.414)\end{array}$ & $\begin{array}{c}2.280^{* * *} \\
(0.534)\end{array}$ & $\begin{array}{l}-1.955 \\
(1.426)\end{array}$ & $\begin{array}{c}0.012 \\
(0.172)\end{array}$ \\
\hline Insurance $\times$ Norms & $\begin{array}{l}0.844^{*} \\
(0.462)\end{array}$ & $\begin{array}{c}0.119 \\
(0.127)\end{array}$ & $\begin{array}{c}0.207 \\
(0.140)\end{array}$ & $\begin{array}{c}0.236 \\
(0.167)\end{array}$ & $\begin{array}{l}-0.026 \\
(0.215)\end{array}$ & $\begin{array}{l}1.146^{* *} \\
(0.575)\end{array}$ & $\begin{array}{c}0.032 \\
(0.076)\end{array}$ \\
\hline Morality $\times$ Norms & $\begin{array}{c}1.082^{* * *} \\
(0.404)\end{array}$ & $\begin{array}{l}0.198^{*} \\
(0.110)\end{array}$ & $\begin{array}{l}0.218^{*} \\
(0.121)\end{array}$ & $\begin{array}{l}0.256^{*} \\
(0.144)\end{array}$ & $\begin{array}{l}-0.093 \\
(0.186)\end{array}$ & $\begin{array}{c}0.747 \\
(0.500)\end{array}$ & $\begin{array}{l}-0.031 \\
(0.068)\end{array}$ \\
\hline Social Capital $\times$ Norms & $\begin{array}{c}0.490 \\
(0.528)\end{array}$ & $\begin{array}{c}0.190 \\
(0.144)\end{array}$ & $\begin{array}{c}0.185 \\
(0.159)\end{array}$ & $\begin{array}{c}0.405^{* *} \\
(0.189)\end{array}$ & $\begin{array}{l}-0.108 \\
(0.244)\end{array}$ & $\begin{array}{l}0.527 \\
(0.652)\end{array}$ & $\begin{array}{l}0.167^{*} \\
(0.091)\end{array}$ \\
\hline Heuristics $\times$ Norms & $\begin{array}{c}0.537 \\
(0.387)\end{array}$ & $\begin{array}{l}0.194^{*} \\
(0.106)\end{array}$ & $\begin{array}{l}0.244^{* *} \\
(0.117)\end{array}$ & $\begin{array}{l}0.240^{*} \\
(0.139)\end{array}$ & $\begin{array}{c}0.082 \\
(0.180)\end{array}$ & $\begin{array}{c}0.639 \\
(0.483)\end{array}$ & $\begin{array}{l}-0.012 \\
(0.066)\end{array}$ \\
\hline Tradition $\times$ Norms & $\begin{array}{c}0.306 \\
(0.420)\end{array}$ & $\begin{array}{l}-0.070 \\
(0.116)\end{array}$ & $\begin{array}{c}0.067 \\
(0.128)\end{array}$ & $\begin{array}{c}0.075 \\
(0.152)\end{array}$ & $\begin{array}{c}0.040 \\
(0.196)\end{array}$ & $\begin{array}{c}0.453 \\
(0.528)\end{array}$ & $\begin{array}{c}0.049 \\
(0.072)\end{array}$ \\
\hline Social Identity $\times$ Norms & $\begin{array}{c}0.537 \\
(0.331)\end{array}$ & $\begin{array}{l}0.105 \\
(0.090)\end{array}$ & $\begin{array}{c}0.132 \\
(0.099)\end{array}$ & $\begin{array}{l}0.196^{*} \\
(0.118)\end{array}$ & $\begin{array}{c}0.072 \\
(0.153)\end{array}$ & $\begin{array}{l}0.680^{*} \\
(0.411)\end{array}$ & $\begin{array}{c}0.015 \\
(0.058)\end{array}$ \\
\hline Insurance $\times$ Endorsement & $\begin{array}{c}0.356 \\
(0.476)\end{array}$ & $\begin{array}{l}0.078 \\
(0.129)\end{array}$ & $\begin{array}{c}0.109 \\
(0.142)\end{array}$ & $\begin{array}{c}0.022 \\
(0.169)\end{array}$ & $\begin{array}{c}0.141 \\
(0.218)\end{array}$ & $\begin{array}{c}0.673 \\
(0.582)\end{array}$ & $\begin{array}{l}-0.057 \\
(0.078)\end{array}$ \\
\hline Morality $\times$ Endorsement & $\begin{array}{l}0.831^{*} \\
(0.431)\end{array}$ & $\begin{array}{c}0.030 \\
(0.117)\end{array}$ & $\begin{array}{c}0.197 \\
(0.129)\end{array}$ & $\begin{array}{l}0.271^{*} \\
(0.153)\end{array}$ & $\begin{array}{l}-0.003 \\
(0.197)\end{array}$ & $\begin{array}{l}0.953^{*} \\
(0.530)\end{array}$ & $\begin{array}{l}-0.079 \\
(0.070)\end{array}$ \\
\hline Social Capital $\times$ Endorsement & $\begin{array}{c}0.994^{* *} \\
(0.457)\end{array}$ & $\begin{array}{c}0.099 \\
(0.125)\end{array}$ & $\begin{array}{c}0.170 \\
(0.138)\end{array}$ & $\begin{array}{c}0.265 \\
(0.165)\end{array}$ & $\begin{array}{l}-0.156 \\
(0.213)\end{array}$ & $\begin{array}{l}1.139^{* *} \\
(0.572)\end{array}$ & $\begin{array}{l}-0.043 \\
(0.075)\end{array}$ \\
\hline Tradition $\times$ Endorsement & $\begin{array}{c}0.261 \\
(0.421)\end{array}$ & $\begin{array}{l}0.086 \\
(0.115)\end{array}$ & $\begin{array}{l}0.089 \\
(0.127)\end{array}$ & $\begin{array}{l}-0.059 \\
(0.152)\end{array}$ & $\begin{array}{l}-0.286 \\
(0.196)\end{array}$ & $\begin{array}{c}0.562 \\
(0.523)\end{array}$ & $\begin{array}{c}0.014 \\
(0.071)\end{array}$ \\
\hline Social Identity $\times$ Endorsement & $\begin{array}{c}0.520 \\
(0.334)\end{array}$ & $\begin{array}{c}0.127 \\
(0.091)\end{array}$ & $\begin{array}{c}0.142 \\
(0.101)\end{array}$ & $\begin{array}{c}0.117 \\
(0.120)\end{array}$ & $\begin{array}{c}0.197 \\
(0.155)\end{array}$ & $\begin{array}{c}0.323 \\
(0.415)\end{array}$ & $\begin{array}{l}-0.051 \\
(0.058)\end{array}$ \\
\hline Insurance $\times$ Control & $\begin{array}{c}0.400 \\
(0.456)\end{array}$ & $\begin{array}{l}-0.093 \\
(0.123)\end{array}$ & $\begin{array}{l}-0.079 \\
(0.136)\end{array}$ & $\begin{array}{c}0.040 \\
(0.162)\end{array}$ & $\begin{array}{l}-0.080 \\
(0.208)\end{array}$ & $\begin{array}{c}0.074 \\
(0.570)\end{array}$ & $\begin{array}{l}-0.100 \\
(0.079)\end{array}$ \\
\hline Morality $\times$ Control & $\begin{array}{c}0.446 \\
(0.409)\end{array}$ & $\begin{array}{l}0.068 \\
(0.113)\end{array}$ & $\begin{array}{l}0.048 \\
(0.124)\end{array}$ & $\begin{array}{c}0.140 \\
(0.147)\end{array}$ & $\begin{array}{c}0.080 \\
(0.190)\end{array}$ & $\begin{array}{c}0.816 \\
(0.511)\end{array}$ & $\begin{array}{c}0.067 \\
(0.068)\end{array}$ \\
\hline Social Capital $\times$ Control & $\begin{array}{c}0.364 \\
(0.471)\end{array}$ & $\begin{array}{c}0.051 \\
(0.127)\end{array}$ & $\begin{array}{l}0.180 \\
(0.141)\end{array}$ & $\begin{array}{c}0.247 \\
(0.167)\end{array}$ & $\begin{array}{l}-0.234 \\
(0.216)\end{array}$ & $\begin{array}{c}0.921 \\
(0.580)\end{array}$ & $\begin{array}{l}-0.113 \\
(0.079)\end{array}$ \\
\hline Tradition $\times$ Control & $\begin{array}{c}0.215 \\
(0.419)\end{array}$ & $\begin{array}{l}-0.064 \\
(0.116)\end{array}$ & $\begin{array}{c}0.006 \\
(0.128)\end{array}$ & $\begin{array}{l}-0.041 \\
(0.152)\end{array}$ & $\begin{array}{l}-0.143 \\
(0.196)\end{array}$ & $\begin{array}{c}0.605 \\
(0.526)\end{array}$ & $\begin{array}{c}0.001 \\
(0.071)\end{array}$ \\
\hline Social Identity $\times$ Control & $\begin{array}{l}-0.036 \\
(0.328)\end{array}$ & $\begin{array}{c}0.125 \\
(0.090)\end{array}$ & $\begin{array}{c}0.010 \\
(0.099)\end{array}$ & $\begin{array}{l}-0.002 \\
(0.118)\end{array}$ & $\begin{array}{l}-0.036 \\
(0.152)\end{array}$ & $\begin{array}{c}0.184 \\
(0.408)\end{array}$ & $\begin{array}{c}0.007 \\
(0.058)\end{array}$ \\
\hline Observations & 1604 & 1687 & 1688 & 1688 & 1688 & 1655 & 1152 \\
\hline$R^{2}$ & 0.360 & 0.100 & 0.203 & 0.298 & 0.086 & 0.337 & 0.070 \\
\hline$F$ & 17.827 & 3.719 & 8.492 & 14.209 & 3.149 & 16.644 & 1.684 \\
\hline
\end{tabular}

Table G28: Interaction Model: Treatment $\times$ Religious Motivations

Omitted Category $=$ Heuristics $\times$ Control 


\begin{tabular}{|c|c|c|c|c|c|c|c|}
\hline & Thermometer & Policy: Hand Washing & Policy: Distancing & Policy: Mask Wearing & Policy: Prayer & Would Click & Clicked \\
\hline Intercept & $\begin{array}{c}0.701 \\
(1.166)\end{array}$ & $\begin{array}{c}3.462^{* * *} \\
(0.317)\end{array}$ & $\begin{array}{c}3.229^{* * *} \\
(0.349)\end{array}$ & $\begin{array}{c}1.594^{* * *} \\
(0.415)\end{array}$ & $2.136^{* * *}$ & $\begin{array}{l}-1.350 \\
(1.430)\end{array}$ & $\begin{array}{c}0.014 \\
(0.171)\end{array}$ \\
\hline \multirow{2}{*}{ Insurance $\times$ Norms } & 0.628 & 0.183 & 0.201 & 0.277 & 0.117 & 0.541 & 0.030 \\
\hline & $(0.471)$ & $(0.131)$ & $(0.145)$ & $(0.172)$ & $(0.222)$ & $(0.592)$ & $(0.075)$ \\
\hline \multirow{2}{*}{ Morality $\times$ Norms } & $0.867^{* *}$ & $0.262^{* *}$ & $0.212^{*}$ & $0.296^{* *}$ & 0.050 & 0.142 & -0.033 \\
\hline & $(0.417)$ & $(0.115)$ & $(0.127)$ & $(0.151)$ & $(0.195)$ & $(0.522)$ & $(0.067)$ \\
\hline \multirow[t]{2}{*}{ Social Capital $\times$ Norms } & 0.274 & $0.254^{*}$ & 0.179 & $0.446 * *$ & 0.035 & -0.078 & $0.166^{*}$ \\
\hline & $(0.536)$ & $(0.148)$ & $(0.164)$ & $(0.195)$ & $(0.251)$ & $(0.669)$ & $(0.090)$ \\
\hline \multirow{2}{*}{ Heuristics $\times$ Norms } & 0.322 & $0.258^{* *}$ & $0.238^{*}$ & $0.280^{*}$ & 0.225 & 0.034 & -0.014 \\
\hline & $(0.400)$ & $(0.111)$ & $(0.123)$ & $(0.146)$ & $(0.189)$ & $(0.506)$ & $(0.065)$ \\
\hline \multirow[t]{2}{*}{ Tradition $\times$ Norms } & 0.091 & -0.006 & 0.061 & 0.116 & 0.184 & -0.152 & 0.048 \\
\hline & $(0.432)$ & $(0.121)$ & $(0.133)$ & $(0.159)$ & $(0.205)$ & $(0.549)$ & $(0.070)$ \\
\hline \multirow[t]{2}{*}{ Social Identity $\times$ Norms } & 0.322 & $0.169^{*}$ & 0.126 & $0.236^{*}$ & 0.215 & 0.075 & 0.013 \\
\hline & $(0.346)$ & $(0.096)$ & $(0.106)$ & $(0.126)$ & $(0.163)$ & $(0.437)$ & $(0.056)$ \\
\hline \multirow[t]{2}{*}{ Insurance $\times$ Endorsement } & 0.141 & 0.142 & 0.103 & 0.063 & 0.284 & 0.068 & -0.058 \\
\hline & $(0.485)$ & $(0.133)$ & $(0.147)$ & $(0.174)$ & $(0.225)$ & $(0.600)$ & $(0.076)$ \\
\hline \multirow[t]{2}{*}{ Morality $\times$ Endorsement } & 0.615 & 0.093 & 0.191 & $0.312^{* *}$ & 0.140 & 0.347 & -0.080 \\
\hline & $(0.441)$ & $(0.121)$ & $(0.134)$ & $(0.159)$ & $(0.205)$ & $(0.549)$ & $(0.068)$ \\
\hline \multirow{2}{*}{ Social Capital $\times$ Endorsement } & $0.778^{*}$ & 0.163 & 0.164 & $0.305^{*}$ & -0.012 & 0.533 & -0.045 \\
\hline & $(0.464)$ & $(0.129)$ & $(0.143)$ & $(0.170)$ & $(0.219)$ & $(0.587)$ & $(0.073)$ \\
\hline \multirow{2}{*}{ Heuristics $\times$ Endorsement } & 0.046 & 0.132 & 0.055 & 0.051 & 0.207 & -0.183 & 0.009 \\
\hline & $(0.382)$ & $(0.107)$ & $(0.118)$ & $(0.140)$ & $(0.181)$ & $(0.485)$ & $(0.063)$ \\
\hline \multirow{2}{*}{ Tradition $\times$ Endorsement } & 0.045 & 0.150 & 0.083 & -0.018 & -0.143 & -0.043 & 0.012 \\
\hline & $(0.433)$ & $(0.120)$ & $(0.133)$ & $(0.158)$ & $(0.204)$ & $(0.544)$ & $(0.070)$ \\
\hline \multirow[t]{2}{*}{ Social Identity $\times$ Endorsement } & 0.305 & $0.191^{*}$ & 0.136 & 0.158 & $0.340^{* *}$ & -0.282 & -0.053 \\
\hline & $(0.349)$ & $(0.098)$ & $(0.108)$ & $(0.128)$ & $(0.165)$ & $(0.442)$ & $(0.057)$ \\
\hline \multirow[t]{2}{*}{ Insurance $\times$ Control } & 0.185 & -0.030 & -0.085 & 0.081 & 0.064 & -0.531 & -0.102 \\
\hline & $(0.466)$ & $(0.128)$ & $(0.141)$ & $(0.168)$ & $(0.216)$ & $(0.590)$ & $(0.078)$ \\
\hline \multirow[t]{2}{*}{ Morality $\times$ Control } & 0.230 & 0.132 & 0.042 & 0.181 & 0.223 & 0.211 & 0.066 \\
\hline & $(0.421)$ & $(0.118)$ & $(0.129)$ & $(0.154)$ & $(0.199)$ & $(0.534)$ & $(0.067)$ \\
\hline \multirow[t]{2}{*}{ Social Capital $\times$ Control } & 0.148 & 0.115 & 0.174 & $0.287^{*}$ & -0.091 & 0.316 & -0.114 \\
\hline & $(0.482)$ & $(0.132)$ & $(0.146)$ & $(0.174)$ & $(0.224)$ & $(0.601)$ & $(0.078)$ \\
\hline \multirow[t]{2}{*}{ Heuristics $\times$ Control } & -0.215 & 0.064 & -0.006 & 0.041 & 0.143 & -0.605 & -0.001 \\
\hline & $(0.419)$ & $(0.116)$ & $(0.128)$ & $(0.152)$ & $(0.196)$ & $(0.526)$ & $(0.071)$ \\
\hline \multirow[t]{2}{*}{ Social Identity $\times$ Control } & -0.252 & $0.188^{* *}$ & 0.004 & 0.039 & 0.107 & -0.421 & 0.006 \\
\hline & $(0.342)$ & $(0.096)$ & $(0.106)$ & $(0.126)$ & $(0.162)$ & $(0.435)$ & $(0.056)$ \\
\hline Observations & 1604 & 1687 & 1688 & 1688 & 1688 & 1655 & 1152 \\
\hline$R^{2}$ & 0.360 & 0.100 & 0.203 & 0.298 & 0.086 & 0.337 & 0.070 \\
\hline$F$ & 17.827 & 3.719 & 8.492 & 14.209 & 3.149 & 16.644 & 1.684 \\
\hline $\begin{array}{l}\text { Note: Models include all control } \\
{ }^{*} p<0.1,{ }^{* *} p<0.05,{ }^{* * *} p<0.0\end{array}$ & & & & & & & \\
\hline
\end{tabular}

Table G29: Interaction Model: Treatment $\times$ Religious Motivations

Omitted Category $=$ Tradition $\times$ Control 


\begin{tabular}{|c|c|c|c|c|c|c|c|}
\hline & Thermometer & Policy: Hand Washing & Policy: Distancing & Policy: Mask Wearing & Policy: Prayer & Would Click & Clicked \\
\hline Intercept & $\begin{array}{c}1.330 \\
(1.139)\end{array}$ & $\begin{array}{c}3.992^{* * *} \\
(0.308)\end{array}$ & $\begin{array}{c}3.829 * * * \\
(0.337)\end{array}$ & $\begin{array}{c}2.130^{* * *} \\
(0.403)\end{array}$ & $\begin{array}{c}2.923^{* * *} \\
(0.510)\end{array}$ & $\begin{array}{l}-0.778 \\
(1.388)\end{array}$ & $\begin{array}{c}0.074 \\
(0.162)\end{array}$ \\
\hline \multirow[t]{2}{*}{ Control $\times$ Strongly Disagree } & $-2.238^{* *}$ & $-0.869^{* * *}$ & $-0.780^{* * *}$ & $-0.865^{* * *}$ & $-0.998^{* *}$ & -0.955 & -0.149 \\
\hline & $(0.948)$ & $(0.250)$ & $(0.273)$ & $(0.327)$ & $(0.413)$ & $(1.121)$ & $(0.153)$ \\
\hline Endorsement $\times$ Strongly Disagree & $\begin{array}{l}-1.132 \\
(0.861)\end{array}$ & $-1.231^{* * *}$ & $-1.327^{* * *}$ & $-1.412^{* * *}$ & $\begin{array}{l}-0.581 \\
(0.380)\end{array}$ & $-2.163^{* *}$ & 0.059 \\
\hline \multirow{2}{*}{ Norms $\times$ Strongly Disagree } & 0.951 & $-0.512^{* *}$ & -0.137 & $\begin{array}{c}(0.300) \\
0.000\end{array}$ & $\begin{array}{l}(0.380) \\
-0.395\end{array}$ & $\begin{array}{c}(1.031) \\
0.619\end{array}$ & $\begin{array}{l}(0.157) \\
-0.178\end{array}$ \\
\hline & $(0.801)$ & $(0.226)$ & $(0.247)$ & $(0.295)$ & $(0.373)$ & $(1.012)$ & $(0.124)$ \\
\hline \multirow{3}{*}{ Control $\times$ Disagree } & -0.842 & $-0.274^{*}$ & -0.220 & $-0.412^{*}$ & $-0.952 * * *$ & -0.782 & 0.061 \\
\hline & $(0.595)$ & $(0.163)$ & $(0.178)$ & $(0.213)$ & $(0.270)$ & $(0.765)$ & $(0.111)$ \\
\hline & -0.021 & -0.118 & -0.259 & -0.214 & $-0.600^{*}$ & -0.410 & 0.064 \\
\hline \multirow[b]{2}{*}{ Norms $\times$ Disagree } & $(0.661)$ & $(0.186)$ & $(0.203)$ & $(0.243)$ & $(0.307)$ & $(0.834)$ & $(0.117)$ \\
\hline & 0.463 & $-0.468^{* * *}$ & $-0.532^{* * *}$ & -0.134 & $-0.934^{* * *}$ & -0.169 & -0.012 \\
\hline & $(0.515)$ & $(0.138)$ & $(0.151)$ & $(0.181)$ & $(0.229)$ & $(0.621)$ & $(0.085)$ \\
\hline \multirow[t]{2}{*}{ Control $\times$ Neither Agree nor Disagree } & -0.484 & $-0.278^{* * *}$ & $-0.438^{* * *}$ & $-0.342^{* * *}$ & $-0.750 * * *$ & $-0.855^{* *}$ & -0.004 \\
\hline & $(0.336)$ & $(0.092)$ & $(0.101)$ & $(0.121)$ & $(0.153)$ & $(0.415)$ & $(0.053)$ \\
\hline \multirow[t]{2}{*}{ Endorsement $\times$ Neither Agree nor Disagree } & -0.499 & $-0.338^{* * *}$ & $-0.349^{* * *}$ & $-0.476^{* * *}$ & $-0.561^{* * *}$ & $-0.825^{* *}$ & -0.077 \\
\hline & $(0.322)$ & $(0.089)$ & $(0.097)$ & $(0.116)$ & $(0.147)$ & $(0.401)$ & $(0.054)$ \\
\hline \multirow[t]{2}{*}{ Norms $\times$ Neither Agree nor Disagree } & -0.513 & $-0.228^{* *}$ & $-0.260^{* *}$ & $-0.289^{* *}$ & $-0.522^{* * *}$ & $-1.046^{* *}$ & -0.048 \\
\hline & $(0.343)$ & $(0.094)$ & $(0.103)$ & $(0.123)$ & $(0.156)$ & $(0.426)$ & $(0.056)$ \\
\hline \multirow{2}{*}{ Control $\times$ Agree } & $-0.707^{* * *}$ & $-0.299 * * *$ & $-0.244^{* * *}$ & $-0.325^{* * *}$ & $-0.388^{* * *}$ & $-0.940 * * *$ & $-0.077^{* *}$ \\
\hline & $(0.231)$ & $(0.063)$ & $(0.069)$ & $(0.083)$ & $(0.105)$ & $(0.287)$ & $(0.037)$ \\
\hline \multirow[t]{2}{*}{ Endorsement $\times$ Agree } & -0.330 & $-0.283^{* * *}$ & $-0.253^{* * *}$ & $-0.254^{* * *}$ & $-0.390 * * *$ & $-0.483^{*}$ & $-0.067^{*}$ \\
\hline & $(0.235)$ & $(0.065)$ & $(0.071)$ & $(0.085)$ & $(0.107)$ & $(0.292)$ & $(0.036)$ \\
\hline \multirow[t]{2}{*}{ Norms $\times$ Agree } & $-0.540^{* *}$ & $-0.255^{* * *}$ & $-0.204^{* * *}$ & $-0.268^{* * *}$ & $-0.323^{* * *}$ & $-0.843^{* * *}$ & -0.029 \\
\hline & $(0.235)$ & $(0.064)$ & $(0.070)$ & $(0.084)$ & $(0.106)$ & $(0.291)$ & $(0.037)$ \\
\hline \multirow[t]{2}{*}{ Control $\times$ Stongly Agree } & $-0.704^{* * *}$ & $-0.110^{*}$ & $-0.258^{* * *}$ & $-0.272^{* * * *}$ & -0.023 & $-0.655^{* *}$ & -0.028 \\
\hline & $(0.233)$ & $(0.064)$ & $(0.070)$ & $(0.083)$ & $(0.105)$ & $(0.288)$ & $(0.037)$ \\
\hline \multirow[t]{2}{*}{ Endorsement $\times$ Stongly Agree } & -0.325 & -0.053 & -0.017 & $-0.163^{* *}$ & 0.072 & $-0.647^{* *}$ & $-0.088^{* *}$ \\
\hline & $(0.232)$ & $(0.063)$ & $(0.069)$ & $(0.083)$ & $(0.105)$ & $(0.287)$ & $(0.036)$ \\
\hline Observations & 1675 & 1767 & 1768 & 1768 & 1767 & 1732 & 1208 \\
\hline$R^{2}$ & 0.349 & 0.133 & 0.220 & 0.297 & 0.126 & 0.333 & 0.057 \\
\hline$F$ & 18.976 & 5.724 & 10.535 & 15.836 & 5.409 & 18.253 & 1.529 \\
\hline $\begin{array}{l}\text { Note: Models include all controls. } \\
{ }^{*} p<0.1,{ }^{* *} p<0.05,{ }^{* * *} p<0.01\end{array}$ & & & & & & & \\
\hline
\end{tabular}

Table G30: Interaction Model: Treatment $\times$ Extrinsic / Intrinsic Motivations — Religion Offers Comfort 


\begin{tabular}{|c|c|c|c|c|c|c|c|}
\hline & Thermometer & Policy: Hand Washing & Policy: Distancing & Policy: Mask Wearing & Policy: Prayer & Would Click & Clicked \\
\hline Intercept & $\begin{array}{c}1.382 \\
(1.135)\end{array}$ & $\begin{array}{c}3.815^{* * *} \\
(0.307)\end{array}$ & $\begin{array}{c}3.667^{* * *} \\
(0.336)\end{array}$ & $\begin{array}{c}1.977^{* * *} \\
(0.401)\end{array}$ & $\begin{array}{c}2.799^{* * *} \\
(0.492)\end{array}$ & $\begin{array}{l}-1.286 \\
(1.380)\end{array}$ & $\begin{array}{c}0.070 \\
(0.162)\end{array}$ \\
\hline Control $\times$ Strongly Disagree & $\begin{array}{c}-2.404^{* * *} \\
(0.798)\end{array}$ & $\begin{array}{c}-0.486^{* *} \\
(0.215)\end{array}$ & $\begin{array}{c}-1.233^{* * *} \\
(0.236)\end{array}$ & $\begin{array}{c}-1.426^{* * *} \\
(0.281)\end{array}$ & $\begin{array}{l}-1.256^{* * *} \\
(0.346)\end{array}$ & $\begin{array}{l}-0.842 \\
(1.010)\end{array}$ & $\begin{array}{l}-0.132 \\
(0.155)\end{array}$ \\
\hline Endorsement $\times$ Strongly Disagree & $\begin{array}{l}-1.628 \\
(1.106)\end{array}$ & $\begin{array}{l}-0.844^{* * * *} \\
(0.286)\end{array}$ & $\begin{array}{c}-0.731^{* *} \\
(0.313)\end{array}$ & $\begin{array}{c}-1.160^{* * * *} \\
(0.374)\end{array}$ & $\begin{array}{c}-1.730^{* * * *} \\
(0.459)\end{array}$ & $\begin{array}{l}-2.358^{*} \\
(1.291)\end{array}$ & $\begin{array}{l}-0.084 \\
(0.222)\end{array}$ \\
\hline Norms $\times$ Strongly Disagree & $\begin{array}{c}0.096 \\
(1.442)\end{array}$ & $\begin{array}{c}0.259 \\
(0.407)\end{array}$ & $\begin{array}{c}0.042 \\
(0.446)\end{array}$ & $\begin{array}{l}-0.336 \\
(0.532)\end{array}$ & $\begin{array}{l}-0.668 \\
(0.654)\end{array}$ & $\begin{array}{l}0.927 \\
(1.826)\end{array}$ & $\begin{array}{l}-0.169 \\
(0.245)\end{array}$ \\
\hline Control $\times$ Disagree & $\begin{array}{l}-0.471 \\
(0.479)\end{array}$ & $\begin{array}{c}-0.286^{* *} \\
(0.135)\end{array}$ & $\begin{array}{l}-0.145 \\
(0.148)\end{array}$ & $\begin{array}{l}-0.269 \\
(0.176)\end{array}$ & $\begin{array}{l}-1.505^{* * *} \\
(0.217)\end{array}$ & $\begin{array}{l}-0.883 \\
(0.605)\end{array}$ & $\begin{array}{l}-0.108 \\
(0.077)\end{array}$ \\
\hline Endorsement $\times$ Disagree & $\begin{array}{l}-0.278 \\
(0.483)\end{array}$ & $\begin{array}{c}-0.333^{* *} \\
(0.130)\end{array}$ & $\begin{array}{l}-0.248^{*} \\
(0.143)\end{array}$ & $\begin{array}{c}-0.398^{* *} \\
(0.171)\end{array}$ & $\begin{array}{c}-1.336^{* * *} \\
(0.209)\end{array}$ & $\begin{array}{l}-0.276 \\
(0.593)\end{array}$ & $\begin{array}{l}-0.145^{*} \\
(0.078)\end{array}$ \\
\hline Norms $\times$ Disagree & $\begin{array}{l}-0.935^{*} \\
(0.483)\end{array}$ & $\begin{array}{c}-0.522^{* * *} \\
(0.134)\end{array}$ & $\begin{array}{r}-0.246^{*} \\
(0.147)\end{array}$ & $\begin{array}{l}-0.248 \\
(0.175)\end{array}$ & $\begin{array}{l}-1.295^{* * *} \\
(0.215)\end{array}$ & $\begin{array}{l}-0.964 \\
(0.601)\end{array}$ & $\begin{array}{c}0.041 \\
(0.080)\end{array}$ \\
\hline Control $\times$ Neither Agree nor Disagree & $\begin{array}{c}-1.106^{* * *} \\
(0.320)\end{array}$ & $\begin{array}{c}-0.397^{* * *} \\
(0.089)\end{array}$ & $\begin{array}{l}-0.347^{* * *} \\
(0.097)\end{array}$ & $\begin{array}{c}-0.335^{* * *} \\
(0.116)\end{array}$ & $\begin{array}{c}-0.699 * * * \\
(0.142)\end{array}$ & $\begin{array}{l}-0.753^{*} \\
(0.402)\end{array}$ & $\begin{array}{l}-0.056 \\
(0.052)\end{array}$ \\
\hline Endorsement $\times$ Neither Agree nor Disagree & $\begin{array}{l}-0.592^{*} \\
(0.312)\end{array}$ & $\begin{array}{c}-0.308^{* * *} \\
(0.087)\end{array}$ & $\begin{array}{c}-0.343^{* * *} \\
(0.095)\end{array}$ & $\begin{array}{c}-0.364^{* * *} \\
(0.114)\end{array}$ & $\begin{array}{c}-0.775^{* * *} \\
(0.140)\end{array}$ & $\begin{array}{l}-0.640 \\
(0.391)\end{array}$ & $\begin{array}{l}-0.032 \\
(0.051)\end{array}$ \\
\hline Norms $\times$ Neither Agree nor Disagree & $\begin{array}{l}-0.498 \\
(0.326)\end{array}$ & $\begin{array}{c}-0.254^{* * *} \\
(0.089)\end{array}$ & $\begin{array}{c}-0.291^{* * *} \\
(0.098)\end{array}$ & $\begin{array}{l}-0.164 \\
(0.117)\end{array}$ & $\begin{array}{c}-0.759^{* * *} \\
(0.143)\end{array}$ & $\begin{array}{l}-0.448 \\
(0.405)\end{array}$ & $\begin{array}{l}-0.023 \\
(0.051)\end{array}$ \\
\hline Control $\times$ Agree & $\begin{array}{c}-0.586^{* *} \\
(0.236)\end{array}$ & $\begin{array}{c}-0.166^{* *} \\
(0.065)\end{array}$ & $\begin{array}{l}-0.259^{* * * *} \\
(0.071)\end{array}$ & $\begin{array}{l}-0.231^{* * * *} \\
(0.084)\end{array}$ & $\begin{array}{c}-0.321^{* * *} \\
(0.104)\end{array}$ & $\begin{array}{l}-0.421 \\
(0.292)\end{array}$ & $\begin{array}{l}-0.061 \\
(0.038)\end{array}$ \\
\hline Endorsement $\times$ Agree & $\begin{array}{l}-0.308 \\
(0.232)\end{array}$ & $\begin{array}{c}-0.150^{* *} \\
(0.064)\end{array}$ & $\begin{array}{c}-0.244^{* * *} \\
(0.070)\end{array}$ & $\begin{array}{c}-0.171^{* *} \\
(0.084)\end{array}$ & $\begin{array}{l}-0.189^{*} \\
(0.103)\end{array}$ & $\begin{array}{l}0.060 \\
(0.288)\end{array}$ & $\begin{array}{l}-0.063^{*} \\
(0.037)\end{array}$ \\
\hline Norms $\times$ Agree & $\begin{array}{r}-0.441^{*} \\
(0.238)\end{array}$ & $\begin{array}{c}-0.109^{*} \\
(0.065)\end{array}$ & $\begin{array}{c}-0.176^{* *} \\
(0.071)\end{array}$ & $\begin{array}{c}-0.197^{* *} \\
(0.085)\end{array}$ & $\begin{array}{c}-0.237^{* *} \\
(0.105)\end{array}$ & $\begin{array}{l}-0.097 \\
(0.296)\end{array}$ & $\begin{array}{l}-0.041 \\
(0.039)\end{array}$ \\
\hline Control $\times$ Stongly Agree & $\begin{array}{l}-0.740^{* * *} \\
(0.232)\end{array}$ & $\begin{array}{l}-0.065 \\
(0.064)\end{array}$ & $\begin{array}{c}-0.191^{* * *} \\
(0.070)\end{array}$ & $\begin{array}{c}-0.260^{* * *} \\
(0.084)\end{array}$ & $\begin{array}{l}-0.038 \\
(0.103)\end{array}$ & $\begin{array}{l}-0.368 \\
(0.289)\end{array}$ & $\begin{array}{c}0.009 \\
(0.039)\end{array}$ \\
\hline Endorsement $\times$ Stongly Agree & $\begin{array}{l}-0.406^{*} \\
(0.239)\end{array}$ & $\begin{array}{l}-0.021 \\
(0.066)\end{array}$ & $\begin{array}{c}0.021 \\
(0.072)\end{array}$ & $\begin{array}{c}-0.197^{* *} \\
(0.086)\end{array}$ & $\begin{array}{c}0.107 \\
(0.105)\end{array}$ & $\begin{array}{r}-0.493^{*} \\
(0.296)\end{array}$ & $\begin{array}{c}-0.074^{*} \\
(0.040)\end{array}$ \\
\hline Observations & 1677 & 1769 & 1770 & 1770 & 1769 & 1734 & 1210 \\
\hline$R^{2}$ & 0.351 & 0.119 & 0.217 & 0.296 & 0.173 & 0.332 & 0.056 \\
\hline$F$ & 19.166 & 5.039 & 10.357 & 15.786 & 7.811 & 18.188 & 1.511 \\
\hline $\begin{array}{l}\text { Note: Models include all controls. } \\
{ }^{*} p<0.1,{ }^{* *} p<0.05,{ }^{* * *} p<0.01\end{array}$ & & & & & & & \\
\hline
\end{tabular}

Table G31: Interaction Model: Treatment $\times$ Extrinsic / Intrinsic Motivations — Takes Religious Approach to Life 


\begin{tabular}{|c|c|c|c|c|c|c|c|}
\hline & Thermometer & Policy: Hand Washing & Policy: Distancing & Policy: Mask Wearing & Policy: Prayer & Would Click & Clicked \\
\hline Intercept & $\begin{array}{c}1.535 \\
(1.197)\end{array}$ & $\begin{array}{c}3.971^{* * *} \\
(0.327)\end{array}$ & $\begin{array}{c}3.704^{* * *} \\
(0.358)\end{array}$ & $\begin{array}{c}2.075^{* * *} \\
(0.424)\end{array}$ & $\begin{array}{c}2.917^{* * *} \\
(0.542)\end{array}$ & $\begin{array}{l}-0.381 \\
(1.459)\end{array}$ & $\begin{array}{c}0.129 \\
(0.171)\end{array}$ \\
\hline Control $\times$ Strongly Disagree & $\begin{array}{c}-1.502^{* * * *} \\
(0.465)\end{array}$ & $\begin{array}{c}-0.315^{* *} \\
(0.127)\end{array}$ & $\begin{array}{c}-0.483^{* * *} \\
(0.138)\end{array}$ & $\begin{array}{c}-0.666^{* * *} \\
(0.164)\end{array}$ & $\begin{array}{c}-0.425^{* *} \\
(0.210)\end{array}$ & $\begin{array}{l}-2.175^{* * *} \\
(0.571)\end{array}$ & $\begin{array}{l}0.017 \\
(0.074)\end{array}$ \\
\hline Endorsement $\times$ Strongly Disagree & $\begin{array}{l}-0.551 \\
(0.475)\end{array}$ & $\begin{array}{c}-0.338^{* * *} \\
(0.130)\end{array}$ & $\begin{array}{l}-0.203 \\
(0.142)\end{array}$ & $\begin{array}{c}-0.398^{* *} \\
(0.169)\end{array}$ & $\begin{array}{l}-0.253 \\
(0.215)\end{array}$ & $\begin{array}{c}-1.261^{* *} \\
(0.587)\end{array}$ & $\begin{array}{l}-0.045 \\
(0.074)\end{array}$ \\
\hline Norms $\times$ Strongly Disagree & $\begin{array}{l}-0.012 \\
(0.489)\end{array}$ & $\begin{array}{c}-0.271^{* *} \\
(0.133)\end{array}$ & $\begin{array}{l}-0.221 \\
(0.145)\end{array}$ & $\begin{array}{r}-0.321^{*} \\
(0.172)\end{array}$ & $\begin{array}{c}-0.575^{* * *} \\
(0.220)\end{array}$ & $\begin{array}{l}-0.672 \\
(0.600)\end{array}$ & $\begin{array}{l}-0.100 \\
(0.075)\end{array}$ \\
\hline Control $\times$ Disagree & $\begin{array}{l}-0.662 \\
(0.435)\end{array}$ & $\begin{array}{c}-0.286^{* *} \\
(0.119)\end{array}$ & $\begin{array}{c}-0.272^{* *} \\
(0.130)\end{array}$ & $\begin{array}{c}-0.386^{* *} \\
(0.154)\end{array}$ & $\begin{array}{c}-0.558^{* * *} \\
(0.196)\end{array}$ & $\begin{array}{c}-1.319^{* *} \\
(0.536)\end{array}$ & $\begin{array}{l}-0.090 \\
(0.066)\end{array}$ \\
\hline Endorsement $\times$ Disagree & $\begin{array}{l}-0.621 \\
(0.432)\end{array}$ & $\begin{array}{c}-0.254^{* *} \\
(0.118)\end{array}$ & $\begin{array}{l}-0.187 \\
(0.129)\end{array}$ & $\begin{array}{c}-0.360^{* *} \\
(0.153)\end{array}$ & $\begin{array}{c}-0.476^{* *} \\
(0.195)\end{array}$ & $\begin{array}{l}-1.521^{* * *} \\
(0.531)\end{array}$ & $\begin{array}{c}-0.150^{* *} \\
(0.065)\end{array}$ \\
\hline Norms $\times$ Disagree & $\begin{array}{l}-0.421 \\
(0.431)\end{array}$ & $\begin{array}{r}-0.224^{*} \\
(0.117)\end{array}$ & $\begin{array}{l}-0.140 \\
(0.128)\end{array}$ & $\begin{array}{l}-0.175 \\
(0.152)\end{array}$ & $\begin{array}{c}-0.396^{* *} \\
(0.194)\end{array}$ & $\begin{array}{c}-1.059^{* *} \\
(0.531)\end{array}$ & $\begin{array}{l}-0.050 \\
(0.065)\end{array}$ \\
\hline Control $\times$ Neither Agree nor Disagree & $\begin{array}{c}-1.010^{* *} \\
(0.430)\end{array}$ & $\begin{array}{c}-0.321^{* * *} \\
(0.117)\end{array}$ & $\begin{array}{c}-0.318^{* *} \\
(0.128)\end{array}$ & $\begin{array}{c}-0.422^{* * *} \\
(0.152)\end{array}$ & $\begin{array}{c}-0.595^{* * *} \\
(0.194)\end{array}$ & $\begin{array}{c}-1.446^{* * *} \\
(0.529)\end{array}$ & $\begin{array}{l}-0.082 \\
(0.065)\end{array}$ \\
\hline Endorsement $\times$ Neither Agree nor Disagree & $\begin{array}{l}-0.723^{*} \\
(0.431)\end{array}$ & $\begin{array}{l}-0.354^{* * * *} \\
(0.117)\end{array}$ & $\begin{array}{c}-0.342^{* * * *} \\
(0.128)\end{array}$ & $\begin{array}{l}-0.427^{* * * *} \\
(0.152)\end{array}$ & $\begin{array}{c}-0.428^{* *} \\
(0.194)\end{array}$ & $\begin{array}{c}-1.171^{* *} \\
(0.529)\end{array}$ & $\begin{array}{l}-0.080 \\
(0.065)\end{array}$ \\
\hline Norms $\times$ Neither Agree nor Disagree & $\begin{array}{c}-0.931^{* *} \\
(0.433)\end{array}$ & $\begin{array}{c}-0.338^{* * *} \\
(0.117)\end{array}$ & $\begin{array}{c}-0.320^{* *} \\
(0.128)\end{array}$ & $\begin{array}{c}-0.427^{* * *} \\
(0.152)\end{array}$ & $\begin{array}{c}-0.503^{* * *} \\
(0.195)\end{array}$ & $\begin{array}{l}-1.566^{* * * *} \\
(0.531)\end{array}$ & $\begin{array}{l}-0.026 \\
(0.065)\end{array}$ \\
\hline Control $\times$ Agree & $\begin{array}{c}-1.026^{* *} \\
(0.468)\end{array}$ & $\begin{array}{c}-0.350^{* * * *} \\
(0.128)\end{array}$ & $\begin{array}{c}-0.406^{* * * *} \\
(0.140)\end{array}$ & $\begin{array}{c}-0.413^{* *} \\
(0.165)\end{array}$ & $\begin{array}{l}-0.415^{*} \\
(0.212)\end{array}$ & $\begin{array}{l}-1.035^{*} \\
(0.575)\end{array}$ & $\begin{array}{l}-0.016 \\
(0.071)\end{array}$ \\
\hline Endorsement $\times$ Agree & $\begin{array}{l}-0.345 \\
(0.476)\end{array}$ & $\begin{array}{l}-0.205 \\
(0.130)\end{array}$ & $\begin{array}{l}-0.200 \\
(0.142)\end{array}$ & $\begin{array}{l}-0.250 \\
(0.168)\end{array}$ & $\begin{array}{l}-0.412^{*} \\
(0.215)\end{array}$ & $\begin{array}{l}-0.760 \\
(0.586)\end{array}$ & $\begin{array}{l}-0.083 \\
(0.070)\end{array}$ \\
\hline Norms $\times$ Agree & $\begin{array}{l}-0.592 \\
(0.471)\end{array}$ & $\begin{array}{l}-0.142 \\
(0.127)\end{array}$ & $\begin{array}{l}-0.148 \\
(0.139)\end{array}$ & $\begin{array}{l}-0.211 \\
(0.165)\end{array}$ & $\begin{array}{l}-0.193 \\
(0.211)\end{array}$ & $\begin{array}{l}-0.899 \\
(0.576)\end{array}$ & $\begin{array}{l}-0.003 \\
(0.071)\end{array}$ \\
\hline Control $\times$ Stongly Agree & $\begin{array}{l}-0.830 \\
(0.555)\end{array}$ & $\begin{array}{l}-0.125 \\
(0.148)\end{array}$ & $\begin{array}{l}-0.174 \\
(0.162)\end{array}$ & $\begin{array}{l}-0.167 \\
(0.192)\end{array}$ & $\begin{array}{l}0.285 \\
(0.246)\end{array}$ & $\begin{array}{l}-0.693 \\
(0.678)\end{array}$ & $\begin{array}{l}-0.077 \\
(0.081)\end{array}$ \\
\hline $\begin{array}{l}\text { Endorsement } \times \text { Stongly Agree } \\
\text { Norms } \times \text { Stongly Agree }\end{array}$ & $\begin{array}{l}-0.895 \\
(0.545) \\
\text { NA }\end{array}$ & $\begin{array}{l}-0.042 \\
(0.150) \\
\text { NA }\end{array}$ & $\begin{array}{l}-0.153 \\
(0.164) \\
\text { NA }\end{array}$ & $\begin{array}{c}-0.435^{* *} \\
(0.194) \\
\text { NA }\end{array}$ & $\begin{array}{l}0.148 \\
(0.248) \\
\text { NA }\end{array}$ & $\begin{array}{l}-1.071 \\
(0.669) \\
\text { NA }\end{array}$ & $\begin{array}{l}0.027 \\
(0.083) \\
\text { NA }\end{array}$ \\
\hline Observations & 1677 & 1769 & 1770 & 1770 & 1769 & 1734 & 1209 \\
\hline$R^{2}$ & 0.351 & 0.102 & 0.199 & 0.291 & 0.102 & 0.337 & 0.063 \\
\hline$F$ & 19.202 & 4.273 & 9.323 & 15.339 & 4.273 & 18.609 & 1.691 \\
\hline
\end{tabular}

Table G32: Interaction Model: Treatment $\times$ Extrinsic / Intrinsic Motivations — Enjoys Social Aspects of Church 


\begin{tabular}{|c|c|c|c|c|c|c|c|}
\hline & Thermometer & Policy: Hand Washing & Policy: Distancing & Policy: Mask Wearing & Policy: Prayer & Would Click & Clicked \\
\hline Intercept & $\begin{array}{c}1.437 \\
(1.134)\end{array}$ & $\begin{array}{c}3.898^{* * *} \\
(0.309)\end{array}$ & $\begin{array}{c}3.624^{* * *} \\
(0.340)\end{array}$ & $\begin{array}{c}2.014^{* * *} \\
(0.404)\end{array}$ & $\begin{array}{c}2.706^{* * *} \\
(0.513)\end{array}$ & $\begin{array}{l}-1.321 \\
(1.382)\end{array}$ & $\begin{array}{c}0.039 \\
(0.162)\end{array}$ \\
\hline Control $\times$ Strongly Disagree & $\begin{array}{c}-1.553^{* * *} \\
(0.409)\end{array}$ & $\begin{array}{c}-0.374^{* * *} \\
(0.114)\end{array}$ & $\begin{array}{c}-0.500^{* * *} \\
(0.125)\end{array}$ & $\begin{array}{c}-0.437^{* * *} \\
(0.148)\end{array}$ & $\begin{array}{c}-0.707^{* * *} \\
(0.188)\end{array}$ & $\begin{array}{l}-0.656 \\
(0.510)\end{array}$ & $\begin{array}{l}-0.121^{*} \\
(0.072)\end{array}$ \\
\hline Endorsement $\times$ Strongly Disagree & $\begin{array}{c}0.266 \\
(0.386)\end{array}$ & $\begin{array}{c}-0.540^{* * *} \\
(0.107)\end{array}$ & $\begin{array}{c}-0.426 * * * \\
(0.118)\end{array}$ & $\begin{array}{c}-0.342^{* *} \\
(0.140)\end{array}$ & $\begin{array}{c}-0.599 * * * \\
(0.178)\end{array}$ & $\begin{array}{c}0.664 \\
(0.482)\end{array}$ & $\begin{array}{l}-0.046 \\
(0.060)\end{array}$ \\
\hline Norms $\times$ Strongly Disagree & $\begin{array}{l}-0.512 \\
(0.457)\end{array}$ & $\begin{array}{c}-0.282^{* *} \\
(0.128)\end{array}$ & $\begin{array}{l}-0.076 \\
(0.140)\end{array}$ & $\begin{array}{l}-0.198 \\
(0.167)\end{array}$ & $\begin{array}{c}-0.370^{*} \\
(0.212)\end{array}$ & $\begin{array}{c}0.260 \\
(0.569)\end{array}$ & $\begin{array}{c}0.034 \\
(0.070)\end{array}$ \\
\hline Control $\times$ Disagree & $\begin{array}{c}-0.887^{* *} \\
(0.350)\end{array}$ & $\begin{array}{c}-0.283^{* * *} \\
(0.097)\end{array}$ & $\begin{array}{c}-0.324^{* * *} \\
(0.107)\end{array}$ & $\begin{array}{c}-0.317^{* *} \\
(0.127)\end{array}$ & $\begin{array}{c}-0.519^{* * *} \\
(0.162)\end{array}$ & $\begin{array}{c}-0.997^{* *} \\
(0.443)\end{array}$ & $\begin{array}{l}0.115^{*} \\
(0.061)\end{array}$ \\
\hline Endorsement $\times$ Disagree & $\begin{array}{l}-0.450 \\
(0.347)\end{array}$ & $\begin{array}{c}-0.294^{* * *} \\
(0.096)\end{array}$ & $\begin{array}{l}-0.172 \\
(0.106)\end{array}$ & $\begin{array}{c}-0.283^{* *} \\
(0.126)\end{array}$ & $\begin{array}{c}-0.421^{* * *} \\
(0.160)\end{array}$ & $\begin{array}{l}-0.058 \\
(0.434)\end{array}$ & $\begin{array}{l}-0.034 \\
(0.055)\end{array}$ \\
\hline Norms $\times$ Disagree & $\begin{array}{l}-0.639^{*} \\
(0.347)\end{array}$ & $\begin{array}{c}-0.305^{* * *} \\
(0.095)\end{array}$ & $\begin{array}{l}-0.196^{*} \\
(0.104)\end{array}$ & $\begin{array}{l}-0.214^{*} \\
(0.124)\end{array}$ & $\begin{array}{c}-0.473^{* * *} \\
(0.157)\end{array}$ & $\begin{array}{l}-0.731^{*} \\
(0.429)\end{array}$ & $\begin{array}{l}0.025 \\
(0.058)\end{array}$ \\
\hline Control $\times$ Somewhat Disagree & $\begin{array}{l}-0.298 \\
(0.378)\end{array}$ & $\begin{array}{c}-0.234^{* *} \\
(0.105)\end{array}$ & $\begin{array}{c}-0.191^{*} \\
(0.115)\end{array}$ & $\begin{array}{c}-0.285^{* *} \\
(0.137)\end{array}$ & $\begin{array}{c}-0.447^{* *} \\
(0.175)\end{array}$ & $\begin{array}{c}0.082 \\
(0.466)\end{array}$ & $\begin{array}{l}-0.079 \\
(0.058)\end{array}$ \\
\hline Endorsement $\times$ Somewhat Disagree & $\begin{array}{l}-0.493 \\
(0.372)\end{array}$ & $\begin{array}{c}-0.279^{* * *} \\
(0.103)\end{array}$ & $\begin{array}{l}-0.161 \\
(0.114)\end{array}$ & $\begin{array}{l}-0.232^{*} \\
(0.135)\end{array}$ & $\begin{array}{c}-0.414^{* *} \\
(0.171)\end{array}$ & $\begin{array}{l}-0.534 \\
(0.461)\end{array}$ & $\begin{array}{l}-0.027 \\
(0.059)\end{array}$ \\
\hline Norms $\times$ Somewhat Disagree & $\begin{array}{l}-0.312 \\
(0.435)\end{array}$ & $\begin{array}{l}-0.228^{*} \\
(0.120)\end{array}$ & $\begin{array}{l}-0.204 \\
(0.132)\end{array}$ & $\begin{array}{l}-0.285^{*} \\
(0.157)\end{array}$ & $\begin{array}{c}-0.480^{* *} \\
(0.199)\end{array}$ & $\begin{array}{l}-0.162 \\
(0.542)\end{array}$ & $\begin{array}{l}0.028 \\
(0.072)\end{array}$ \\
\hline Control $\times$ Somewhat Agree & $\begin{array}{c}-0.835^{* *} \\
(0.348)\end{array}$ & $\begin{array}{c}-0.396^{* * * *} \\
(0.098)\end{array}$ & $\begin{array}{c}-0.327^{* * *} \\
(0.107)\end{array}$ & $\begin{array}{c}-0.317^{* *} \\
(0.128)\end{array}$ & $\begin{array}{l}-0.289^{*} \\
(0.162)\end{array}$ & $\begin{array}{l}0.055 \\
(0.436)\end{array}$ & $\begin{array}{l}-0.056 \\
(0.055)\end{array}$ \\
\hline Endorsement $\times$ Somewhat Agree & $\begin{array}{l}-0.482 \\
(0.341)\end{array}$ & $\begin{array}{l}-0.127 \\
(0.096)\end{array}$ & $\begin{array}{l}-0.084 \\
(0.105)\end{array}$ & $\begin{array}{l}-0.191 \\
(0.125)\end{array}$ & $\begin{array}{c}-0.374^{* *} \\
(0.158)\end{array}$ & $\begin{array}{c}0.552 \\
(0.427)\end{array}$ & $\begin{array}{l}-0.019 \\
(0.053)\end{array}$ \\
\hline Norms $\times$ Somewhat Agree & $\begin{array}{c}-1.006^{* * *} \\
(0.337)\end{array}$ & $\begin{array}{c}-0.194^{* *} \\
(0.092)\end{array}$ & $\begin{array}{c}-0.207^{* *} \\
(0.101)\end{array}$ & $\begin{array}{c}-0.364^{* * *} \\
(0.121)\end{array}$ & $\begin{array}{c}-0.327^{* *} \\
(0.153)\end{array}$ & $\begin{array}{l}-0.290 \\
(0.417)\end{array}$ & $\begin{array}{l}-0.056 \\
(0.055)\end{array}$ \\
\hline Control $\times$ Agree & $\begin{array}{c}-0.906^{* * * *} \\
(0.305)\end{array}$ & $\begin{array}{c}-0.252^{* * *} \\
(0.084)\end{array}$ & $\begin{array}{l}-0.130 \\
(0.092)\end{array}$ & $\begin{array}{c}-0.302^{* * * *} \\
(0.109)\end{array}$ & $\begin{array}{l}-0.090 \\
(0.139)\end{array}$ & $\begin{array}{l}-0.387 \\
(0.377)\end{array}$ & $\begin{array}{l}-0.015 \\
(0.050)\end{array}$ \\
\hline Endorsement $\times$ Agree & $\begin{array}{l}-0.994^{* * * *} \\
(0.286)\end{array}$ & $\begin{array}{c}-0.185^{* *} \\
(0.079)\end{array}$ & $\begin{array}{c}-0.183^{* *} \\
(0.087)\end{array}$ & $\begin{array}{c}-0.359^{* * *} \\
(0.104)\end{array}$ & $\begin{array}{l}-0.197 \\
(0.131)\end{array}$ & $\begin{array}{l}-0.501 \\
(0.357)\end{array}$ & $\begin{array}{l}-0.028 \\
(0.046)\end{array}$ \\
\hline Norms $\times$ Agree & $\begin{array}{l}-0.432 \\
(0.283)\end{array}$ & $\begin{array}{c}-0.223^{* * *} \\
(0.079)\end{array}$ & $\begin{array}{l}-0.156^{*} \\
(0.086)\end{array}$ & $\begin{array}{r}-0.192^{*} \\
(0.103)\end{array}$ & $\begin{array}{l}-0.148 \\
(0.130)\end{array}$ & $\begin{array}{c}0.179 \\
(0.352)\end{array}$ & $\begin{array}{l}-0.024 \\
(0.044)\end{array}$ \\
\hline Control $\times$ Strongly Agree & $\begin{array}{c}-0.714^{* * *} \\
(0.228)\end{array}$ & $\begin{array}{l}-0.089 \\
(0.063)\end{array}$ & $\begin{array}{c}-0.205^{* * *} \\
(0.069)\end{array}$ & $\begin{array}{c}-0.298^{* * *} \\
(0.082)\end{array}$ & $\begin{array}{l}-0.141 \\
(0.104)\end{array}$ & $\begin{array}{l}-0.507^{*} \\
(0.283)\end{array}$ & $\begin{array}{l}-0.020 \\
(0.038)\end{array}$ \\
\hline Endorsement $\times$ Strongly Agree & $\begin{array}{l}-0.348 \\
(0.238)\end{array}$ & $\begin{array}{l}-0.069 \\
(0.066)\end{array}$ & $\begin{array}{l}-0.081 \\
(0.072)\end{array}$ & $\begin{array}{c}-0.220^{* *} \\
(0.086)\end{array}$ & $\begin{array}{c}0.137 \\
(0.109)\end{array}$ & $\begin{array}{l}-0.556^{*} \\
(0.293)\end{array}$ & $\begin{array}{c}-0.102^{* *} \\
(0.040)\end{array}$ \\
\hline Observations & 1680 & 1772 & 1773 & 1773 & 1772 & 1737 & 1211 \\
\hline$R^{2}$ & 0.354 & 0.120 & 0.204 & 0.289 & 0.111 & 0.335 & 0.063 \\
\hline$F$ & 18.255 & 4.812 & 8.996 & 14.290 & 4.375 & 17.347 & 1.588 \\
\hline
\end{tabular}

Table G33: Interaction Model: Treatment $\times$ Regularly Attends Church 


\begin{tabular}{|c|c|c|c|c|c|c|c|}
\hline & Thermometer & Policy: Hand Washing & Policy: Distancing & Policy: Mask Wearing & Policy: Prayer & Would Click & Clicked \\
\hline \multirow[t]{2}{*}{ Intercept } & 1.313 & $3.911^{* * *}$ & $3.676^{* * *}$ & $1.955^{* * *}$ & $2.848^{* * *}$ & -1.468 & 0.037 \\
\hline & (1.134) & $(0.305)$ & $(0.337)$ & $(0.403)$ & $(0.501)$ & $(1.377)$ & $(0.161)$ \\
\hline \multirow[t]{2}{*}{ Control $\times$ Strongly Disagree } & $-1.877^{* * *}$ & $-0.318^{*}$ & $-0.401^{*}$ & $-0.607^{* *}$ & -0.413 & -1.276 & -0.140 \\
\hline & $(0.677)$ & $(0.190)$ & $(0.210)$ & $(0.251)$ & $(0.312)$ & $(0.856)$ & $(0.116)$ \\
\hline \multirow{2}{*}{ Endorsement $\times$ Strongly Disagree } & 0.156 & $-0.792^{* * *}$ & $-0.608^{* *}$ & -0.365 & -0.432 & 0.666 & $0.200^{*}$ \\
\hline & $(0.774)$ & $(0.218)$ & $(0.240)$ & $(0.287)$ & $(0.357)$ & $(0.980)$ & $(0.120)$ \\
\hline \multirow[t]{2}{*}{ Norms $\times$ Strongly Disagree } & -0.661 & 0.077 & -0.397 & -0.444 & 0.363 & -0.668 & -0.182 \\
\hline & $(1.239)$ & $(0.349)$ & $(0.384)$ & $(0.460)$ & $(0.572)$ & $(1.568)$ & $(0.197)$ \\
\hline \multirow[t]{2}{*}{ Control $\times$ Disagree } & 0.283 & -0.174 & 0.039 & -0.313 & $-2.080^{* * *}$ & -0.121 & 0.311 \\
\hline & $(1.128)$ & $(0.318)$ & $(0.350)$ & $(0.419)$ & $(0.521)$ & $(1.565)$ & $(0.240)$ \\
\hline \multirow[t]{2}{*}{ Endorsement $\times$ Disagree } & -1.581 & $-1.169 * * *$ & $-1.237 * * *$ & $-0.954^{* *}$ & -0.616 & 1.190 & 0.054 \\
\hline & $(1.111)$ & $(0.287)$ & $(0.316)$ & $(0.378)$ & $(0.470)$ & $(1.406)$ & $(0.153)$ \\
\hline \multirow[t]{2}{*}{ Norms $\times$ Disagree } & 1.520 & $-1.048^{* * *}$ & $-0.593^{* *}$ & -0.347 & $-1.177^{* * *}$ & $2.471^{* *}$ & 0.137 \\
\hline & $(0.945)$ & $(0.249)$ & $(0.274)$ & $(0.328)$ & $(0.408)$ & $(1.119)$ & $(0.131)$ \\
\hline \multirow[t]{2}{*}{ Control $\times$ Somewhat Disagree } & -0.339 & $-0.544^{* * *}$ & $-0.341^{*}$ & -0.201 & $-0.884^{* * *}$ & 0.115 & $-0.165^{*}$ \\
\hline & $(0.621)$ & $(0.170)$ & $(0.187)$ & $(0.224)$ & $(0.278)$ & $(0.785)$ & $(0.089)$ \\
\hline \multirow{2}{*}{ Endorsement $\times$ Somewhat Disagree } & -0.369 & $-0.786^{* * *}$ & $-0.689 * * *$ & $-0.658^{* *}$ & $-1.186^{* * *}$ & 0.990 & -0.134 \\
\hline & $(0.764)$ & $(0.215)$ & $(0.237)$ & $(0.283)$ & $(0.352)$ & $(0.967)$ & $(0.111)$ \\
\hline \multirow[t]{2}{*}{ Norms $\times$ Somewhat Disagree } & 1.099 & 0.054 & -0.376 & 0.277 & $-1.015^{* * *}$ & 0.300 & -0.200 \\
\hline & $(0.953)$ & $(0.238)$ & $(0.262)$ & $(0.314)$ & $(0.390)$ & $(1.069)$ & $(0.124)$ \\
\hline \multirow[t]{2}{*}{ Control $\times$ Somewhat Agree } & $-0.753^{* *}$ & $-0.362^{* * *}$ & $-0.324^{* * *}$ & $-0.236^{* *}$ & $-0.702^{* * *}$ & -0.575 & -0.013 \\
\hline & $(0.300)$ & $(0.082)$ & $(0.090)$ & $(0.108)$ & $(0.134)$ & $(0.374)$ & $(0.048)$ \\
\hline \multirow[t]{2}{*}{ Endorsement $\times$ Somewhat Agree } & -0.344 & $-0.235^{* * *}$ & $-0.185^{*}$ & $-0.190^{*}$ & $-0.911^{* * *}$ & -0.352 & -0.052 \\
\hline & $(0.319)$ & $(0.088)$ & $(0.096)$ & $(0.115)$ & $(0.143)$ & $(0.394)$ & $(0.050)$ \\
\hline \multirow[t]{2}{*}{ Norms $\times$ Somewhat Agree } & $-0.839^{* *}$ & $-0.304^{* * *}$ & $-0.301 * * *$ & -0.186 & $-0.578^{* * *}$ & $-0.737^{*}$ & -0.051 \\
\hline & $(0.360)$ & $(0.098)$ & $(0.108)$ & $(0.129)$ & $(0.160)$ & $(0.442)$ & $(0.055)$ \\
\hline \multirow[t]{2}{*}{ Control $\times$ Agree } & $-0.537^{* *}$ & $-0.190 * * *$ & $-0.162^{* *}$ & $-0.233^{* *}$ & $-0.264^{* *}$ & -0.190 & -0.027 \\
\hline & $(0.249)$ & $(0.069)$ & $(0.076)$ & $(0.091)$ & $(0.113)$ & $(0.311)$ & $(0.040)$ \\
\hline \multirow[t]{2}{*}{ Endorsement $\times$ Agree } & -0.344 & $-0.187 * * *$ & $-0.239 * * *$ & $-0.212^{* *}$ & $-0.346 * * *$ & 0.076 & -0.041 \\
\hline & $(0.242)$ & $(0.067)$ & $(0.073)$ & $(0.088)$ & $(0.109)$ & $(0.302)$ & $(0.038)$ \\
\hline \multirow[t]{2}{*}{ Norms $\times$ Agree } & $-0.693^{* * *}$ & $-0.159^{* *}$ & $-0.135^{*}$ & $-0.195^{* *}$ & $-0.396^{* * *}$ & -0.325 & 0.033 \\
\hline & $(0.242)$ & $(0.066)$ & $(0.073)$ & $(0.087)$ & $(0.109)$ & $(0.303)$ & $(0.040)$ \\
\hline \multirow[t]{2}{*}{ Control $\times$ Strongly Agree } & $-0.753^{* * *}$ & -0.047 & $-0.210^{* * *}$ & $-0.236^{* * *}$ & -0.079 & $-0.586^{* * *}$ & -0.006 \\
\hline & $(0.198)$ & $(0.054)$ & $(0.060)$ & $(0.071)$ & $(0.089)$ & $(0.246)$ & $(0.033)$ \\
\hline \multirow[t]{2}{*}{ Endorsement $\times$ Strongly Agree } & $-0.375^{*}$ & -0.021 & -0.016 & $-0.156^{* *}$ & 0.118 & $-0.554^{* *}$ & $-0.062^{*}$ \\
\hline & $(0.197)$ & $(0.054)$ & $(0.060)$ & $(0.071)$ & $(0.089)$ & $(0.244)$ & $(0.032)$ \\
\hline Observations & 1681 & 1772 & 1773 & 1773 & 1772 & 1737 & 1211 \\
\hline$R^{2}$ & 0.353 & 0.138 & 0.214 & 0.290 & 0.146 & 0.334 & 0.064 \\
\hline$F$ & 18.183 & 5.612 & 9.553 & 14.347 & 6.017 & 17.288 & 1.630 \\
\hline $\begin{array}{l}\text { e: Models include all controls. } \\
<0.1,{ }^{* *} p<0.05,{ }^{* * *} p<0.01\end{array}$ & & & & & & & \\
\hline
\end{tabular}

Table G34: Interaction Model: Treatment $\times$ Spiritual Values 


\begin{tabular}{|c|c|c|c|c|c|c|c|}
\hline & Thermometer & Policy: Hand Washing & Policy: Distancing & Policy: Mask Wearing & Policy: Prayer & Would Click & Clicked \\
\hline Intercept & $\begin{array}{c}0.962 \\
(1.140)\end{array}$ & $\begin{array}{c}3.854^{* * *} \\
(0.310)\end{array}$ & $\begin{array}{c}3.550^{* * *} \\
(0.340)\end{array}$ & $\begin{array}{c}1.839^{* * *} \\
(0.405)\end{array}$ & $\begin{array}{c}2.952^{* * *} \\
(0.500)\end{array}$ & $\begin{array}{l}-1.409 \\
(1.381)\end{array}$ & $\begin{array}{c}0.091 \\
(0.162)\end{array}$ \\
\hline Control $\times$ Strongly Disagree & $\begin{array}{c}-1.366^{* *} \\
(0.659)\end{array}$ & $\begin{array}{r}-0.347^{*} \\
(0.187)\end{array}$ & $\begin{array}{c}-0.598^{* * *} \\
(0.205)\end{array}$ & $\begin{array}{c}-0.769^{* * *} \\
(0.244)\end{array}$ & $\begin{array}{c}-0.649^{* *} \\
(0.301)\end{array}$ & $\begin{array}{l}-1.149 \\
(0.830)\end{array}$ & $\begin{array}{l}-0.134 \\
(0.117)\end{array}$ \\
\hline Endorsement $\times$ Strongly Disagree & $\begin{array}{c}0.319 \\
(0.640)\end{array}$ & $\begin{array}{c}-0.479 * * * \\
(0.181)\end{array}$ & $\begin{array}{c}-0.506^{* *} \\
(0.199)\end{array}$ & $\begin{array}{l}-0.451^{*} \\
(0.237)\end{array}$ & $\begin{array}{c}-0.566^{*} \\
(0.292)\end{array}$ & $\begin{array}{l}0.415 \\
(0.807)\end{array}$ & $\begin{array}{c}0.050 \\
0.098)\end{array}$ \\
\hline Norms $\times$ Strongly Disagree & $\begin{array}{c}0.145 \\
(1.019)\end{array}$ & $\begin{array}{c}0.123 \\
(0.289)\end{array}$ & $\begin{array}{l}-0.147 \\
(0.318)\end{array}$ & $\begin{array}{l}-0.123 \\
(0.378)\end{array}$ & $\begin{array}{l}-0.354 \\
(0.467)\end{array}$ & $\begin{array}{l}0.435 \\
(1.285)\end{array}$ & $\begin{array}{l}-0.183 \\
(0.154)\end{array}$ \\
\hline Control $\times$ Disagree & $\begin{array}{c}0.610 \\
(1.138)\end{array}$ & $\begin{array}{l}-0.571^{*} \\
(0.323)\end{array}$ & $\begin{array}{c}0.104 \\
(0.355)\end{array}$ & $\begin{array}{l}-0.213 \\
(0.422)\end{array}$ & $\begin{array}{c}-1.666^{* * *} \\
(0.521)\end{array}$ & $\begin{array}{l}-0.817 \\
(1.570)\end{array}$ & $\begin{array}{l}-0.189 \\
(0.198)\end{array}$ \\
\hline Endorsement $\times$ Disagree & $\begin{array}{l}-0.008 \\
(0.684)\end{array}$ & $\begin{array}{l}-0.165 \\
(0.187)\end{array}$ & $\begin{array}{l}-0.221 \\
(0.206)\end{array}$ & $\begin{array}{l}-0.213 \\
(0.244)\end{array}$ & $\begin{array}{c}-1.126^{* * *} \\
(0.302)\end{array}$ & $\begin{array}{l}1.268 \\
(0.832)\end{array}$ & $\begin{array}{c}0.085 \\
(0.095)\end{array}$ \\
\hline Norms $\times$ Disagree & $\begin{array}{l}1.032 \\
(0.674)\end{array}$ & $\begin{array}{c}-0.383^{* *} \\
(0.185)\end{array}$ & $\begin{array}{l}-0.347^{*} \\
(0.203)\end{array}$ & $\begin{array}{l}0.086 \\
(0.242)\end{array}$ & $\begin{array}{c}-0.965^{* * *} \\
(0.298)\end{array}$ & $\begin{array}{l}1.193 \\
(0.849)\end{array}$ & $\begin{array}{l}-0.040 \\
(0.095)\end{array}$ \\
\hline Control $\times$ Somewhat Disagree & $\begin{array}{l}-0.211 \\
(0.533)\end{array}$ & $\begin{array}{c}-0.438^{* * *} \\
(0.144)\end{array}$ & $\begin{array}{l}-0.292^{*} \\
(0.158)\end{array}$ & $\begin{array}{l}-0.226 \\
(0.188)\end{array}$ & $\begin{array}{c}-0.943^{* * *} \\
(0.232)\end{array}$ & $\begin{array}{l}0.360 \\
(0.662)\end{array}$ & $\begin{array}{l}-0.106 \\
(0.081)\end{array}$ \\
\hline Endorsement $\times$ Somewhat Disagree & $\begin{array}{l}-0.482 \\
(0.552)\end{array}$ & $\begin{array}{c}-0.532^{* * *} \\
(0.157)\end{array}$ & $\begin{array}{c}-0.441^{* *} \\
(0.172)\end{array}$ & $\begin{array}{l}-0.222 \\
(0.204)\end{array}$ & $\begin{array}{c}-1.152^{* * *} \\
(0.252)\end{array}$ & $\begin{array}{l}-0.920 \\
(0.695)\end{array}$ & $\begin{array}{l}-0.108 \\
(0.092)\end{array}$ \\
\hline Norms $\times$ Somewhat Disagree & $\begin{array}{l}1.237^{* *} \\
(0.590)\end{array}$ & $\begin{array}{c}-0.390^{* *} \\
(0.167)\end{array}$ & $\begin{array}{l}-0.108 \\
(0.184)\end{array}$ & $\begin{array}{c}0.147 \\
(0.218)\end{array}$ & $\begin{array}{c}-0.828^{* * *} \\
(0.270)\end{array}$ & $\begin{array}{l}1.526^{* *} \\
(0.743)\end{array}$ & $\begin{array}{c}0.062 \\
(0.087)\end{array}$ \\
\hline Control $\times$ Somewhat Agree & $\begin{array}{c}-0.565^{* *} \\
(0.274)\end{array}$ & $\begin{array}{c}-0.320^{* * *} \\
(0.076)\end{array}$ & $\begin{array}{c}-0.185^{* *} \\
(0.084)\end{array}$ & $\begin{array}{l}-0.161 \\
(0.099)\end{array}$ & $\begin{array}{c}-0.746^{* * *} \\
(0.123)\end{array}$ & $\begin{array}{c}-0.753^{* *} \\
(0.341)\end{array}$ & $\begin{array}{l}-0.053 \\
(0.045)\end{array}$ \\
\hline Endorsement $\times$ Somewhat Agree & $\begin{array}{l}-0.109 \\
(0.286)\end{array}$ & $\begin{array}{c}-0.190^{* *} \\
(0.079)\end{array}$ & $\begin{array}{c}-0.178^{* *} \\
(0.087)\end{array}$ & $\begin{array}{l}-0.095 \\
(0.103)\end{array}$ & $\begin{array}{c}-0.786^{* * *} \\
(0.128)\end{array}$ & $\begin{array}{c}0.000 \\
(0.356)\end{array}$ & $\begin{array}{l}-0.041 \\
(0.045)\end{array}$ \\
\hline Norms $\times$ Somewhat Agree & $\begin{array}{l}-0.263 \\
(0.289)\end{array}$ & $\begin{array}{c}-0.233^{* * *} \\
(0.079)\end{array}$ & $\begin{array}{l}-0.130 \\
(0.087)\end{array}$ & $\begin{array}{l}-0.048 \\
(0.103)\end{array}$ & $\begin{array}{c}-0.678^{* * *} \\
(0.128)\end{array}$ & $\begin{array}{l}-0.543 \\
(0.356)\end{array}$ & $\begin{array}{l}-0.054 \\
(0.046)\end{array}$ \\
\hline Control $\times$ Agree & $\begin{array}{c}-0.507^{* *} \\
(0.252)\end{array}$ & $\begin{array}{c}-0.180^{* *} \\
(0.070)\end{array}$ & $\begin{array}{c}-0.215^{* * *} \\
(0.077)\end{array}$ & $\begin{array}{c}-0.247^{* * * *} \\
(0.091)\end{array}$ & $\begin{array}{c}-0.324^{* * * *} \\
(0.113)\end{array}$ & $\begin{array}{l}-0.389 \\
(0.313)\end{array}$ & $\begin{array}{l}-0.050 \\
(0.041)\end{array}$ \\
\hline Endorsement $\times$ Agree & $\begin{array}{l}-0.196 \\
(0.261)\end{array}$ & $\begin{array}{l}-0.130^{*} \\
(0.072)\end{array}$ & $\begin{array}{c}-0.157^{* *} \\
(0.079)\end{array}$ & $\begin{array}{l}-0.127 \\
(0.094)\end{array}$ & $\begin{array}{l}-0.104 \\
(0.116)\end{array}$ & $\begin{array}{l}-0.095 \\
(0.323)\end{array}$ & $\begin{array}{r}-0.082^{*} \\
(0.043)\end{array}$ \\
\hline Norms $\times$ Agree & $\begin{array}{c}-0.512^{* *} \\
(0.261)\end{array}$ & $\begin{array}{l}-0.112 \\
(0.072)\end{array}$ & $\begin{array}{l}-0.124 \\
(0.079)\end{array}$ & $\begin{array}{l}-0.177^{*} \\
(0.094)\end{array}$ & $\begin{array}{l}-0.209^{*} \\
(0.116)\end{array}$ & $\begin{array}{l}-0.481 \\
(0.322)\end{array}$ & $\begin{array}{l}-0.009 \\
(0.043)\end{array}$ \\
\hline Control × Strongly Agree & $\begin{array}{l}-0.601^{* * *} \\
(0.218)\end{array}$ & $\begin{array}{l}-0.024 \\
(0.060)\end{array}$ & $\begin{array}{c}-0.190^{* * *} \\
(0.066)\end{array}$ & $\begin{array}{c}-0.187^{* *} \\
(0.078)\end{array}$ & $\begin{array}{l}0.020 \\
(0.097)\end{array}$ & $\begin{array}{l}-0.448^{*} \\
(0.270)\end{array}$ & $\begin{array}{c}0.005 \\
(0.036)\end{array}$ \\
\hline Endorsement $\times$ Strongly Agree & $\begin{array}{l}-0.297 \\
(0.214)\end{array}$ & $\begin{array}{l}-0.075 \\
(0.059)\end{array}$ & $\begin{array}{l}-0.024 \\
(0.065)\end{array}$ & $\begin{array}{c}-0.194^{* *} \\
(0.077)\end{array}$ & $\begin{array}{c}0.079 \\
(0.096)\end{array}$ & $\begin{array}{c}-0.597^{* *} \\
(0.265)\end{array}$ & $\begin{array}{c}-0.079^{* *} \\
(0.035)\end{array}$ \\
\hline Observations & 1681 & 1773 & 1774 & 1774 & 1773 & 1738 & 1212 \\
\hline$R^{2}$ & 0.351 & 0.118 & 0.202 & 0.288 & 0.156 & 0.336 & 0.060 \\
\hline$F$ & 17.996 & 4.692 & 8.885 & 14.248 & 6.486 & 17.458 & 1.526 \\
\hline
\end{tabular}

Table G35: Interaction Model: Treatment $\times$ Americans Should be More Religious 
H Who already knew our treatment information?

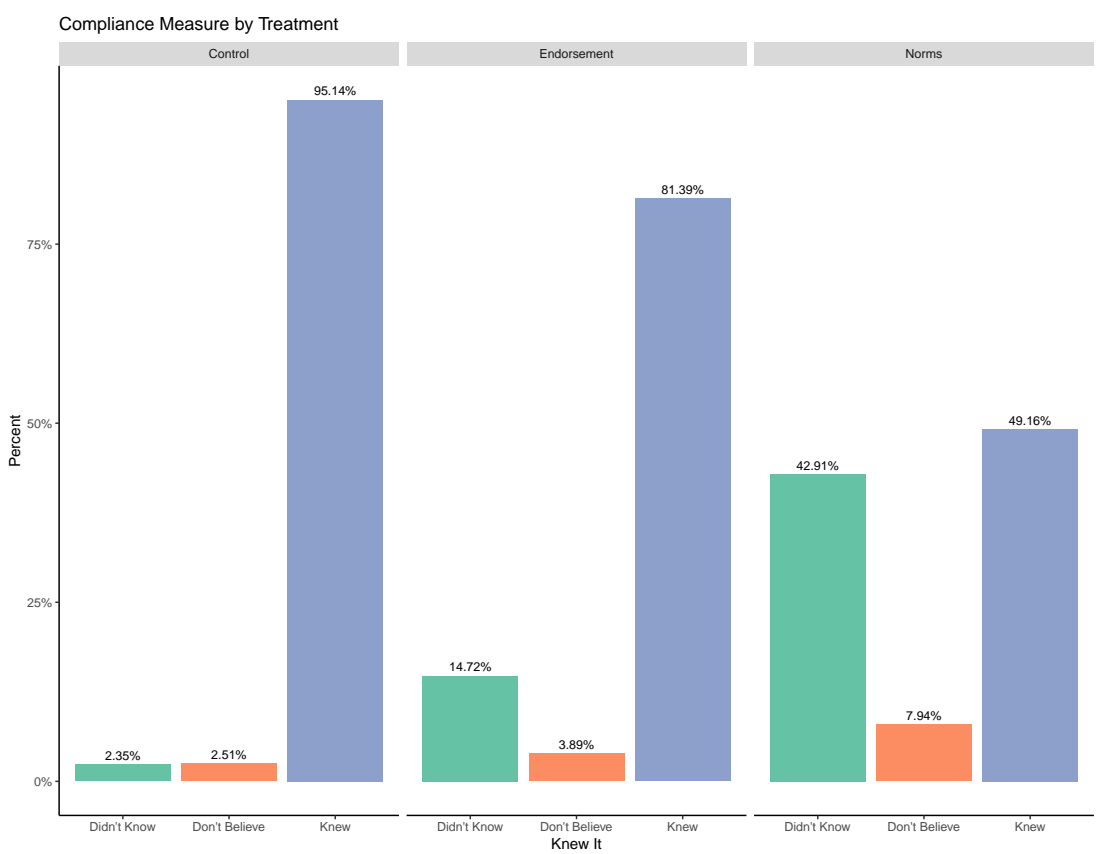

Figure H13: Proportion of respondents who already knew, did not already know, or did not believe the information provided by the treatments 


\section{Pre-analysis plan}

This section offers a screenshot of the study's anonymized pre-analysis plan. 


\title{
Can Religion Increase Compliance with COviD-19 Prevention Measures?
}

Pre-Analysis Plan

July 21, 2020

\begin{abstract}
Many local and national politicians disagree on which public health policies they support to mitigate the ongoing COVID-19 pandemic. In the absence of political consensus on how best to respond to the virus, some individuals turn to other sources of guidance. Drawing from insights from the social and behavioral sciences, we ask whether religious communities effectively influence individuals' beliefs, attitudes, and behaviors with regard to COVID-19 public health guidelines. We field a survey experiment among White Evangelicals in the United States - one of the least likely groups to adhere to social distancing guidelinesto test whether religious elites on one hand, and ingroup norms on the other, can shape White Evangelical beliefs and attitudes toward mask-wearing. The results of our study will help inform strategies for most effectively communicating critical public health guidelines to skeptical groups.
\end{abstract}




\section{Introduction}

As of 1 July 2020, the COVID-19 pandemic has claimed over 500,000 lives and infected more than 10.4 million people worldwide, putting this public health emergency on track to be the most deadly in over a century. ${ }^{1}$ One of the most striking features of the ongoing pandemic is the absence of clear and consistent public health recommendations by local and national governments. In the United States, for example, the Centers for Disease Control and Prevention recommends such measures as hand washing, physical distancing, and mask wearing, though local jurisdictions throughout the country do not uniformly condone or enforce these policies. Indeed, states diverge from CDC guidelines even on reporting their case numbers. ${ }^{2}$ U.S. President Donald Trump has himself provided inconsistent messages on the necessity and efficacy of these measures.

Without clear governmental guidelines on COVID-19 prevention, religious leaders throughout the world have stepped in to either promote or denounce various public health and safety measures. ${ }^{3}$ Alternatively, individuals may turn to norms within their social groups for guidance on appropriate behavior. This project investigates the extent to which religious groups - through the influence of their leaders and/or the impact of ingroup social norms - can shape individual compliance with COVID-19 public health guidelines.

The influence of religious elites on individual-level attitudes and behavior is well-documented in the social science literature. Scholars find that religious elites play an outsized role in individual support for democracy, ${ }^{4}$ political participation, ${ }^{5}$ and voting behavior. ${ }^{6}$ In the domain of public health, scholars note the tremendous influence of religious leaders in both encouraging and discouraging compliance in anti-HIV and anti-Malarial initiatives throughout sub-Saharan Africa and Latin America. ${ }^{7}$ These findings suggest that religious figures have the capacity to shift both attitudes and behavioral practices among congregants, which leads to our first research question: (RQ1) To what extent does the endorsement of religious elites influence co-religionists' compliance with standard COVID-19 prevention measures?

Alternatively, individuals may take a cue not from their religious leader but from what they perceive to be acceptable norms of behavior within their religious group. Ingroup norms are a powerful motivator of individual behavior in many other $\operatorname{contexts}^{8}$, leading to our second research question: (RQ2) To what extent do social norms within a religious group influence an individual's compliance with standard COVID19 prevention measures? Although we expect that both elite endorsements and social norms will impact individual preferences and behavior, and we expect that these effects will significantly differ from each other, we do not have any prior about which effect will be larger.

Additionally, this project seeks to understand the mechanisms driving the above effects. Specifically, it considers two possible mechanisms - religiosity and religious motivations - that could enhance or reduce the impact of religious elites and/or social norms on individual compliance with COVID-19 prevention measures: (RQ3) To what extent does religiosity increase or decrease the effect of elite endorsements and/or social norms on individual compliance with CoviD-19 prevention measures? (RQ4) To what extent do various

\footnotetext{
${ }^{1}$ World Economic Forum, "COVID-19: What you need to know about coronavirus on 1 July"; accessed online on 2 July 2020. Available at: https://www.weforum.org/agenda/2020/07/covid-19-need-to-know-coronavirus-1-july-pandemic/.

${ }^{2}$ Madeline Holcombe, "More Than Half of States may be Undercounting Coronavirus Cases by not Following CDC Guidelines," CNN, 9 June 2020. Available at: https://www.cnn.com/2020/06/09/health/us-coronavirus-tuesday/index.html.

${ }^{3}$ Bryan Schonfeld and Sam Winter-Levy, Who can Convince Americans to Follow the Science on Coronavirus? Religious Leaders., The Washington Post, 8 May 2020. Available at: https://www.washingtonpost.com/politics/2020/05/08/ who-can-convince-americans-follow-science-coronavirus-religious-leaders/.

${ }^{4}$ Smith (2019)

${ }^{5}$ McClendon and Riedl (2019)

${ }^{6}$ Green (2010)

${ }^{7}$ Surur and Kaba (2000); Garcia and Parker (2011); Muñoz-Laboy et al. (2011); Mfaume et al. (1997)

${ }^{8}$ Habyarimana et al. (2009); Paluck (2011); Paluck and Shepherd (2012)
} 
religious motivations increase or decrease the effect of elite endorsements and/or social norms on individual compliance with COVID-19 prevention measures?

To measure religious motivations, we rely first on the extrinsic/intrinsic motivation scale first proposed by Allport and Ross (1967) and further refined by Gorsuch and McPherson (1989). ${ }^{9}$ We also develop our own taxonomy of religious motivations drawing from the rich social scientific literature on social identity.

We measure religiosity following Wilkes, Burnett, and Howell (1986):

1. To what extent do you agree with the following statements:

- I go to Church regularly

- Spiritual values are more important than material things

- If Americans were more religious, this would be a better country

2. Please evaluate your own feelings of religiousness. Are you very religious, moderately religious, slightly religious, not at all religious, or anti-religious?

We measure the explicit / implicit religious motivation scale following Allport and Ross (1967) and Gorsuch and McPherson (1989):

1. To what extent do you agree or disagree with the following statements?

- What religion offers me most is comfort in times of trouble and sorrow (extrinsic personal)

- My whole approach to life is based on my religion (intrinsic)

- I go to church mainly because I enjoy seeing people I know there (extrinsic social)

We develop our own taxonomy of religious motivation by asking respondents to rank order the below statements from the statement they most agree with to the statement they least agree with:

1. Insurance-based motivation: "My Church is important to me because it helps me in times of personal or financial need."

2. Morality-based motivation: "My Church is important to me because following the advice of my Church is the right thing to do."

3. Social capital-based motivation: "My Church is important to me because that is where I find my family close friends, and/or business partners."

4. Heuristics-based motivation: "My Church is important to me because it makes it easier for me to reach decisions about complicated things."

5. Tradition-based motivation: "My Church is important to me because I was born into it and/or brought up that way."

6. Social identity-based motivation: "My Church is important to me because I identify as an Evangelical Christian."

We test each of these four research questions with the following hypotheses:

\footnotetext{
${ }^{9}$ To economize survey length, we limit our scale to the three questions with highest loading on each of three factors: implicit motivation, explicit personal motivation, and explicit social motivation.
} 
$\left(H_{1}\right)$ Individuals who receive a religious elite message endorsing mask-wearing are more likely to comply with mask-wearing than individuals who do not receive such an endorsement message (RQ1)

$\left(H_{2}\right)$ Individuals who receive an ingroup norm message about mask-wearing are more likely to comply with mask-wearing than individuals who do not receive such an ingroup norm message (RQ2)

$\left(H_{3}\right)$ The effect of the religious elite endorsement message will differ from that of the ingroup norm message (RQ1 and RQ2)

$\left(H_{4}\right)$ Individuals who self-identify as more religious are more likely to respond to both elite endorsement and ingroup norm messages relative to individuals who self-report as less religious (RQ3)

$\left(H_{5}\right)$ Individuals with different religion motivations will respond differently to our treatments (RQ4). Specifically:

$\left(H 5_{1}\right)$ Individuals who self-report intrinsic motivations for religion, and individuals who self-report extrinsic personal motivations for religion, are more likely to respond to the elite endorsement message than individuals who self-report extrinsic social motivations for religion (RQ4 - Gorsuch and McPherson (1989) taxonomy)

$\left(H 5_{2}\right)$ Individuals who self-report intrinsic motivations for religion, and respondents who self-report extrinsic personal motivations for religion are less likely to respond to the ingroup norm message relative to respondents who self-report extrinsic social motivations for religion (RQ4 - Gorsuch and McPherson (1989) taxonomy)

$\left(H 5_{3}\right)$ Individuals who self-report social identity, social capital, and insurance motivations for religion will respond more strongly to the ingroup norm message than other respondents. Individuals who self-report heuristics and morality motivations for religion will respond more strongly to the endorsement message than other respondents (RQ4 - Author taxonomy)

We expect the effect of both treatments to increase with levels of religiosity $(H 4)$ because as the importance of religion in one's life increases, so too should the importance of recommendations of leaders and behavior of co-religious adherents. As to $H 5$, individuals with extrinsic social motivations think of the church as a venue to meet and interact with friends and acquaintances. Someone who values social interactions should be more attentive to social norms because non-compliance might hinder their ability to socialize. Therefore, we expect respondents with extrinsic social motivations to be more likely to respond to the ingroup norm message relative to respondents with intrinsic and extrinsic personal motivations for religion $\left(H 5_{1}\right)$.

Further, individuals with intrinsic motivations for religion base themselves on religious codes of conduct when making life choices. Individuals with extrinsic personal motivations for religion find psychological comfort in their faith. Religious leaders help these individuals interpret religious codes of conduct and vindicate the legitimacy of their faith. Therefore, we expect respondents with intrinsic and extrinsic personal motivations to be more likely to respond to the endorsement message relative to respondents with extrinsic social motivations for religion $\left(H 5_{2}\right)$.

Finally, we expect individuals who self-report social identity, social capital and insurance based motivations for religion to have greater incentives to comply with social norms. Individuals with a social identity motivation see compliance with social norms as signals of who belongs and who does not belong in the group; individuals with social capital motivation value social interactions with co-religious adherents; and 
individuals with an insurance motivation want to secure help in times of need. We also expect individuals who self-report heuristics and morality based motivations to value the role of religious leaders in helping them interpret religious codes of conduct $\left(H 5_{3}\right)$. We have no priors on the behavior of individuals who self-report a tradition-based motivation.

\section{Methods}

The following section outlines a survey experiment in which a sample of Evangelical Christians in the United States are randomly exposed to one of three conditions. In the placebo or control condition, respondents will read a short vignette describing a few facts about COVID-19 transmission. These facts are taken from the CDC website. In the endorsement condition, this same set of facts will be presented with the addition of a statement by Evangelical leader Franklin Graham endorsing mask-wearing. In the social norms condition, the endorsement statement will be replaced by the statement that close to $80 \%$ of White Evangelicals have worn a mask in public. It should be noted that all these statements are true, and that no deception is involved in either of the treatment conditions. Our source for Pastor Graham's statement is a 10 April 2020 interview with the Charlotte Observer. Our source for the statistic that $80 \%$ of White Evangelicals have worn a mask in public comes from the magazine Christianity Today, which cites a CovID-19 Tracking Poll from Data for Progress.

\subsection{Ethics Information}

This study has received an exemption from the [REDACTED]. This design complies with all relevant ethical and human subjects regulations. A written statement of informed consent will be obtained from all participants in this study, and no personally identifying informations will be collected. Respondents will not be incentivized for their participation.

In order to mitigate the possibility of unintended risks to the respondents, the experimental treatment will only endorse public health behaviors that are widely accepted by the medical community to reduce the spread of COVID-19. Further, treatments will not employ any form of deception; all endorsements presented to respondents will be factually accurate and framed in a manner consistent with the religious leader's intent.

\subsection{Design}

To test the hypotheses outlined in Section 1, we conduct a brief online survey and endorsement experiment. The 25 item questionnaire is designed to take no more than 15 minutes to complete.

The survey begins with a short battery of demographic questions to ascertain the respondent's gender identity, age, ideology, social and religious identities, religiosity, as well as the respondent's beliefs pertaining to COVID-19 and information-seeking behavior. We then measure our three conditioning variables: religiosity, the explicit / implicit scale of religious motivation, and our 6-point scale of religious motivation. Respondents

are then randomly assigned, with equal probability $\left(\frac{1}{3}, \frac{1}{3}, \frac{1}{3}\right)$, to treatment and control conditions. For each vignette, we also ask respondents whether they already knew the information, and whether they believe it. These questions are used to ensure respondents are actively paying attention to the treatment, and to measure compliance with treatment. 
We measure three outcomes. A thermometer question asks, on a scale of 1 to 10 , how important the respondent believes wearing a mask in public is for combatting the spread of CoviD-19. This is the outcome measure we use for our power analysis. A second question asks the extent to which the respondent agrees with a set of policies to mitigate the spread of COVID-19. Finally, a behavioral measure asks respondents how likely, on a scale of 1 to 10, they would add their name to a Change.org petition endorsing mask-wearing in public. If respondents answer 5 or higher, they are then given the option to click on the petition URL, which is a decision we can track.

\subsection{Sampling plan}

A sample of 1710 adults (aged 18+) will be collected by the online survey firm Lucid. We plan to enumerate the survey among White Evangelical Christians in the United States. Eventually, we plan to extend this sample to Evangelical Christians in Brazil. This design allows us to draw inferences on one of the least compliant religious groups (Evangelicals) in their home countries. ${ }^{10}$

We estimate the sample size necessary to detect differences between three independent group means at $\alpha=0.05$ and $80 \%$ power. We conservatively assume a small effect size, and thus estimate our sample size using a Cohen's $f$ value of 0.075 . Our analysis suggests that we are sufficiently powered to detect relatively small average treatment effects with a sample size of $n=1692$, equally apportioned between our three comparison groups $\left(n_{i}=564\right)$. Results of our analysis are shown in Figure 1; the vertical dashed line indicates the minimum sample needed to achieve $80 \%$ power with $\sigma_{m}^{2}=0.01$ and $\sigma_{e}^{2}=1.75$.

We also estimate the sample size needed to attain $80 \%$ power for our factorial interaction model (see Equation 4) using a simulation-based approach. In particular, we assume $\beta_{1}=0.75, \beta_{2}=-0.1$, and $\epsilon \sim \mathcal{N}(0,1)$, and simulate the power to test the interaction term at $\beta_{3}=\{0.025,0.05,0.075,0.1,0.125\}$, with sample sizes ranging from 500 to 2250. Our results, shown in Figure 2, indicate that we have sufficient power to detect an effect $\geq 0.075$ with a sample size of at least 1500 respondents.

\subsection{Analysis plan}

\subsubsection{Main Effects}

Our first two hypotheses suggest that respondents in the two treatment groups are significantly more likely than those in the control group to comply with COVID-19 prevention measures. We test these hypotheses by first estimating the difference in treatment and control means for each of our three outcomes of interest using a standard $t$-test and one-way ANOVA model. We then reestimate the treatment effect (ITT) more formally using the following OLS model:

$$
y_{i}=\alpha+\beta T_{i}+\gamma X_{i}+\epsilon_{i}
$$

Where $y_{i}$ is the outcome of interest for respondent $i, \beta$ is the estimated treatment effect, $T_{i}$ is the assignment to treatment, $X_{i}$ is a vector of demographic covariates, and $\epsilon_{i}$ is the error term.

Because we also have a measure of treatment compliance (see Section 2.2), we plan to report the treatment effect for compliers using an instrumental variable approach (Huang 2018). We first estimate:

$$
C_{i}=\alpha+\delta T_{i}+\epsilon_{i}
$$

${ }^{10}$ See this recent analysis by Ryan Burge using data collected by Data for Progress. 
Where $C_{i}$ is our compliance variable and $T_{i}$ is assignment to treatment. $\delta$ gives the compliance rate; $\beta$ from Equation 1 divided by $\delta$ represents that compliance adjusted ITT (Sussman and Hayward 2010). The second stage estimates:

$$
y_{i}=\alpha+\zeta \hat{C}_{i}+\gamma X_{i}+\epsilon_{i}
$$

Where $\hat{C}_{i}$ is the predicted compliance values generated by Equation 2, and $\zeta$ is the treatment effect for compliant respondents.

\subsubsection{Religiosity Mechanism}

We test Hypothesis $H_{4}$ using mediation analysis (Baron and Kenny 1986). Specifically, we estimate:

$$
\begin{aligned}
y_{i} & =\alpha+\beta_{1} T_{i}+\gamma X_{i}+\epsilon_{i} \\
M_{i} & =\alpha+\beta_{2} T_{i}+\gamma X_{i}+\epsilon_{i} \\
y_{i} & =\alpha+\beta_{3} T_{i}+\beta_{4} M_{i}+\gamma X_{i}+\epsilon_{i}
\end{aligned}
$$

Where $T_{i}$ is assignment to treatment, $M_{i}$ is the mediator variable, and $X_{i}$ is a vector of pre-treatment covariates. Equation 4 is functionally equivalent to Equation 1 in Section 2.4.1. Equation 5 allows us to test whether treatment affects the mediator; we expect $\beta_{2} \neq 0$. Finally, Equation 6 tests the religiosity mechanism. If $\beta_{3}$ loses statistical significance with the inclusion of the mediation term (full mediation), or if $\beta_{3} \ll \beta_{1}$ (partial mediation), it would suggest that a mediation effect exists and that religiosity is a reasonable explanation of the effect of treatment on our outcome measures.

Additionally, we estimate the conditional average treatment effect using the following factorial interaction model:

$$
y_{i}=\alpha+\beta_{1} T_{i}+\beta_{2} \text { Religious }_{i}+\beta_{3}\left(T_{i} \times \text { Religious }_{i}\right)+\gamma X_{i}+\epsilon_{i}
$$

Where $T$ is the treatment condition and "Religious" is a measure of religiosity derived from Wilkes et al. (1986). In Equation $4, \beta_{1}$ is the treatment effect at Religious $=0, \beta_{2}$ is the effect of religiosity on our outcome of interest, and $\left(\beta_{1}+\beta_{3}\right)$ is the conditional average treatment effect. Since we hypothesize that those respondents who self-report higher levels of religiosity are more likely to respond to both the endorsement and ingroup norms treatments, we expect $\beta_{3}>0$.

\subsubsection{Religious Motivation Mechanism}

Similar to our tests for the religiosity mechanism described in Section 2.4.2, we employ two strategies to test Hypothesis $H_{5}$. We begin with mediation analysis to determine whether religious motivations might mediate the effects of treatment. We estimate the following:

$$
\begin{aligned}
y_{i} & =\alpha+\beta_{1} T_{i}+\gamma X_{i}+\epsilon_{i} \\
M_{i} & =\alpha+\beta_{2} T_{i}+\gamma X_{i}+\epsilon_{i} \\
y_{i} & =\alpha+\beta_{3} T_{i}+\beta_{4} M_{i}+\gamma X_{i}+\epsilon_{i}
\end{aligned}
$$

Equations 8, 9, and 10 are identical to Equations 4 through 6 above, though here $M_{i}$, our mediation variable, is a set of variables that accounts for the three intrinsic and three extrinsic motivations in our six category religious motivation taxonomy (see Section 1). Again, we expect $\beta_{2} \neq 0$ and $\beta_{3} \ll \beta_{1}$, as this would suggest 
that a mediation effect exists and that religious motivations are a reasonable explanation for the effect of treatment on our outcome measures.

Our second strategy estimates the following interaction model:

$$
y_{i}=\alpha+\beta_{1} T_{i}+\beta_{2} \text { Motivation }_{i}+\beta_{3}\left(T_{i} \times \text { Motivation }_{i}\right)+\gamma X_{i}+\epsilon_{i}
$$

Where "Motivation" is a binary indicator of the respondent's first choice ranking of statements 1 through 6 in Section 1 above, and $\beta_{3}$ is the interaction term. We would interpret a positive and significant $\beta_{3}$ as evidence of the religious motivation mechanism.

\section{Data \& Code Availability}

Raw data and supporting materials will be publicly available upon publication. Replication code will be publicly available (via GitHub) upon publication of manuscript.

\section{Acknowledgements}

The authors acknowledge support from REDACTED. The funders have/had no role in study design, data collection and analysis, the decision to publish, or the preparation of the manuscript.

\section{Author Contributions}

All authors contributed equally to all parts of the study.

\section{Competing Interests}

The authors declare no financial or non-financial competing interests.

\section{References}

Allport, Gordon W. and J. Michael Ross (1967). "Personal Religious Orientation and Prejudice." In: Journal of Personality and Social Psychology 5.4, pp. 432-443.

Baron, Reuben M. and David A. Kenny (Dec. 1986). "The Moderator-Mediator Variable Distinction in Social Psychological Research: Conceptual, Strategic, and Statistical Considerations." In: Journal of Personality and Social Psychology 51.6, pp. 1173-1182.

Burge, Ryan P. (June 23, 2020). "White Evangelicals' Coronavirus Concerns Are Fading Faster." In: Christianity Today.

Garcia, Jonathan and Richard G. Parker (June 1, 2011). "Resource Mobilization for Health Advocacy: AfroBrazilian Religious Organizations and HIV Prevention and Control." In: Social Science 83 Medicine. Part Special Issue: Analysing Global Health Assistance 72.12, pp. 1930-1938.

Gorsuch, Richard L. and Susan E. McPherson (1989). "Intrinsic/Extrinsic Measurement: I/E-Revised and Single-Item Scales." In: Journal for the Scientific Study of Religion 28.3, pp. 348-354. JSTOR: 1386745. 
Green, John C. (Feb. 25, 2010). "Gauging the God Gap: Religion and Voting in US Presidential Elections." In: The Oxford Handbook of American Elections and Political Behavior.

Habyarimana, James et al. (July 30, 2009). Coethnicity: Diversity and the Dilemmas of Collective Action. Russell Sage Foundation. 252 pp.

Holcombe, Madeline (June 9, 2020). "More than Half of States May Be Undercounting Coronavirus Cases by Not Following CDC Guidelines." In: $C N N$.

Huang, Francis L. (Feb. 2018). "Using Instrumental Variable Estimation to Evaluate Randomized Experiments with Imperfect Compliance." In: Practical Assessment, Research \& Evaluation 23.2.

Janes, Théoden (Apr. 10, 2020). "Franklin Graham on Easter amid Pandemic: 'God Never Intended for Man to Have Disease'." In: The Charlotte Observer.

McClendon, Gwyneth H. and Rachel Beatty Riedl (Nov. 14, 2019). From Pews to Politics: Religious Sermons and Political Participation in Africa. Cambridge University Press. 289 pp.

Mfaume, Mfaume Selemani et al. (Aug. 25, 1997). "Mosques against malaria." In: World Health Forum 18.1, pp. 35-38. pmid: 9233062.

Muñoz-Laboy, Miguel et al. (Oct. 1, 2011). "Religious Responses to HIV and AIDS: Understanding the Role of Religious Cultures and Institutions in Confronting the Epidemic." In: Global Public Health 6 (sup2), S127-S131. pmid: 21806421.

Paluck, Elizabeth Levy (Mar. 1, 2011). "Peer Pressure against Prejudice: A High School Field Experiment Examining Social Network Change." In: Journal of Experimental Social Psychology 47.2, pp. 350-358.

Paluck, Elizabeth Levy and Hana Shepherd (2012). "The Salience of Social Referents: A Field Experiment on Collective Norms and Harassment Behavior in a School Social Network." In: Journal of Personality and Social Psychology 103.6, pp. 899-915.

Schonfeld, Bryan and Sam Winter-Levy (May 8, 2020). "Analysis - Who Can Convince Americans to Follow the Science on Coronavirus? Religious Leaders." In: Washington Post.

Smith, Amy Erica (Mar. 28, 2019). Religion and Brazilian Democracy: Mobilizing the People of God. Cambridge University Press. 223 pp.

"Study: Young White Evangelicals Are Way Less Likely to Wear a Mask" (June 23, 2020). In: Relevant Magazine.

Surur, Feiruz and Mirgissa Kaba (2000). "The Role of Religious Leaders in HIV/AIDS Prevention, Control, and Patient Care and Support: A Pilot Project in Jimma Zone." In: Northeast African Studies 7.2, pp. 59-79. JSTOR: 41931343.

Sussman, Jeremy B and Rodney A Hayward (May 4, 2010). "An IV for the RCT: Using Instrumental Variables to Adjust for Treatment Contamination in Randomised Controlled Trials." In: The BMJ 340. pmid: 20442226.

Whiting, Kate (July 1, 2020). What You Need to Know about Coronavirus on 1 July. URL: https://www . weforum . org/agenda/2020/07/covid-19-need-to-know-coronavirus-1-july-pandemic/ (visited on $07 / 21 / 2020$ ).

Wilkes, Robert E., John J. Burnett, and Roy D. Howell (Mar. 1, 1986). "On the Meaning and Measurement of Religiosity in Consumer Research." In: Journal of the Academy of Marketing Science 14.1 (1), pp. 4756 . 


\section{$8 \quad$ Figures}

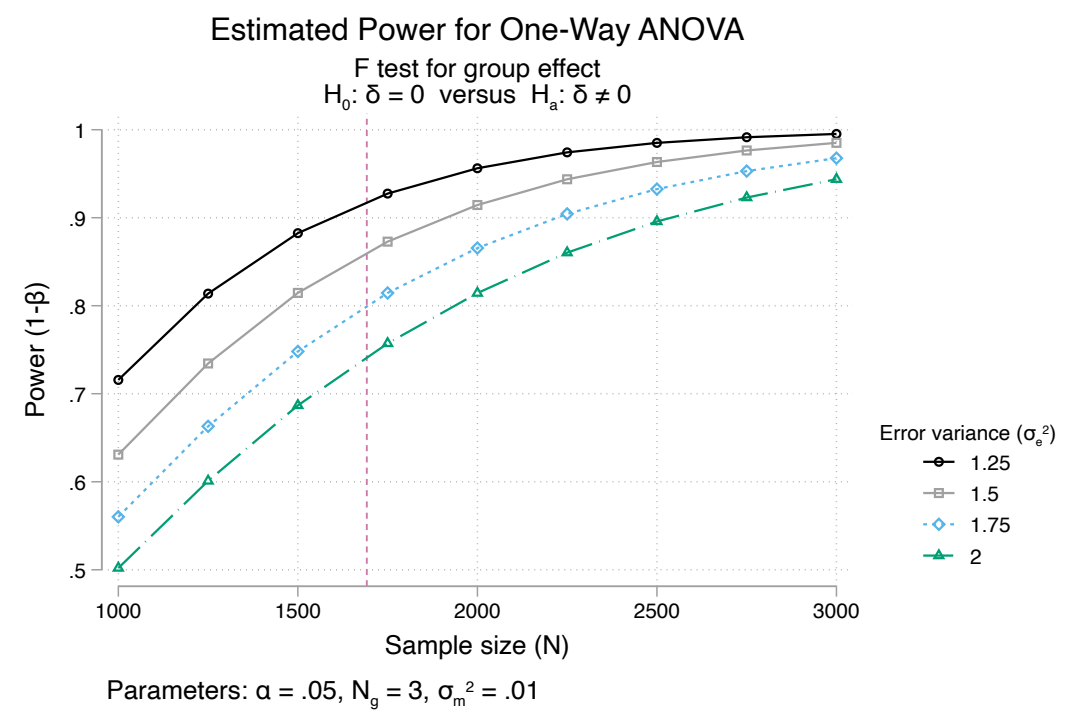

Figure 1: Total sample size needed for $80 \%$ power, difference of means test.

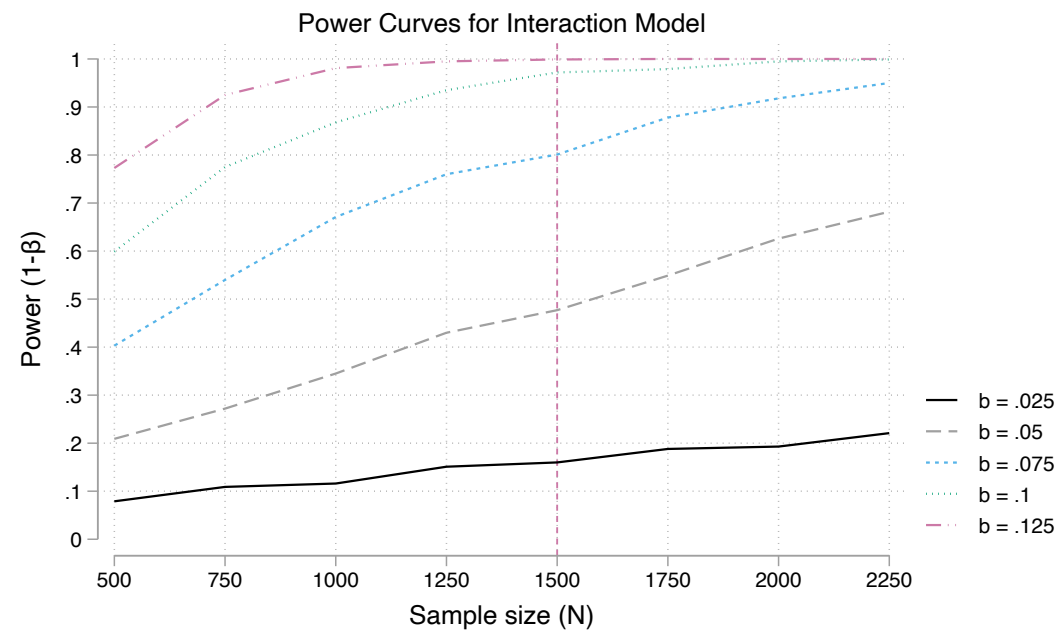

alpha $=.05 ; \mathrm{N}$ of replications per sample and effect size: 1000

Figure 2: Total sample size needed for $80 \%$ power, interaction model. 